\title{
Logarithmic Intertwining Operators and Genus-One Correlation Functions
}

\author{
Francesco Fiordalisi
}

\begin{abstract}
This is the first of two papers in which we study the modular invariance of pseudotraces of logarithmic intertwining operators. We construct and study genus-one correlation functions for logarithmic intertwining operators among generalized modules over a positive-energy and $C_{2}$-cofinite vertex operator algebra $V$. We consider grading-restricted generalized $V$-modules which admit a right action of some associative algebra $P$, and intertwining operators among such modules which commute with the action of $P$ ( $P$-intertwining operators). We obtain duality properties, i.e., suitable associativity and commutativity properties, for $P$-intertwining operators. Using pseudotraces introduced by Miyamoto and studied by Arike, we define formal $q$-traces of products of $P$-intertwining operators, and obtain certain identities for these formal series. This allows us to show that the formal $q$-traces satisfy a system of differential equations with regular singular points, and therefore are absolutely convergent in a suitable region and can be extended to yield multivalued analytic functions, called genus-one correlation functions. Furthermore, we show that the space of solutions of these differential equations is invariant under the action of the modular group.
\end{abstract}

\section{Introduction}

The theory of vertex operator algebra arose independently in mathematics and physics and has been providing deep and remarkable connections between different fields. In mathematics, one of its most spectacular applications was the construction of the "moonshine module", a vertex operator algebra (usually denoted by $V^{\natural}$ ) whose group of automorphisms is the Monster group $\mathbb{M}$, the largest sporadic finite simple group. Noticing patterns relating the dimensions of irreducible modules for the Monster and the Fourier expansion of the modular function $J(q)$, McKay and Thompson conjectured the existence of a "natural" infinite dimensional graded module $V=\coprod_{n=-1}^{\infty} V_{n}$ for $\mathbb{M}$ whose graded dimension

$$
\sum_{n=-1}^{\infty} \operatorname{dim}\left(V_{n}\right) q^{n}
$$


is given exactly by $J(q)$. Additionally, Conway and Norton conjectured that for any element $g$ in the Monster, the series

$$
\left.\sum_{n \in \mathbb{Z}} \operatorname{tr} g\right|_{V_{n}} q^{n}
$$

is the Fourier expansion of a generator of the field of modular functions for some genus zero subgroup of $S L_{2}(\mathbb{R})$. Frenkel, Lepowsky and Meurman constructed a module $V^{\natural}$ for the Monster group in [FLM], proving the McKay-Thompson conjecture and introducing the notion of vertex operator algebra, a variant of Borcherds' notion of vertex algebra $([\mathrm{B}])$. The full Conway-Norton conjecture for $V^{\natural}$ was later proved by Borcherds.

The connection between the theory of vertex operator algebras and the theory of modular functions has deep roots and the solution of the Moonshine conjecture is just a part of it. In the important work [MS1] and [MS2] by Moore and Seiberg, an explicit conjecture on the modular invariance for intertwining operators (called "chiral vertex operators" in [MS1] and [MS2]) was stated. In his Ph.D. thesis [Z] Zhu proved a partial result on the modular invariance conjecture of Moore and Seiberg. Considering a class of "rational" vertex operator algebras satisfying a certain cofiniteness condition, Zhu studied traces of products of $n$ vertex operators associated to irreducible representations, and showed that these formal traces converge and the functions thus obtained (called $n$-point genus-one correlation functions) form a space invariant under the action of the modular group; as direct consequence, he established the modular invariance for the spaces of functions spanned by the graded dimension of the irreducible modules. Zhu's results were later extended by Dong, Li and Mason in DLM2 to include twisted representations; and in Miy1, Miyamoto considered traces of products of vertex operators for modules and at most one intertwining operator.

All these results rely heavily on the use of the commutator formula to obtain recurrence relations for the $n$-point genus-one correlation function in terms of the $n$-1-point functions. Since this formula is not available for general intertwining operators, the methods do not generalize to product of more than one intertwining operator. In [H2], Huang overcame this difficulty and proved the full modular invariance conjecture of Moore and Seiberg; he used commutativity and associativity for intertwining operators to obtain a system of "modular" differential equations, and to obtain genus-one commutativity and associativity properties. This modular invariance result is a crucial ingredient in other important works by Huang, including his proof of the Verlinde conjecture and the rigidity and modularity of the vertex tensor category (see [H4], [H5]) for "rational" vertex operator algebras.

In [M1 Milas considered a class of weak modules for non-rational vertex operator algebras, called "logarithmic modules". These are modules on which the operator $L(0)$ does not necessarily act semisimply, but can be expressed as direct sum of generalized eigenspaces for $L(0)$. Moreover, he introduced and studied "logarithmic intertwining operators" between these modules, that is, intertwining operators which involve (integral) powers of $\log x$ in addition to powers of the formal variable $x$. The theory of such modules and intertwining operators has since been developed (see [HLZ1]-[HLZ8]) and interesting classes of such modules have been constructed (see for instance [M2], [AM1]- AM3]).

Huang conjectured that a full modular invariance result should hold for such classes of 
modules, and that it should play an important role in the study of the properties of logarithmic modules. Before this conjecture was explicitly formulated, a partial result generalizing Zhu's result in the context of logarithmic modules was obtained first by Miyamoto Miy2, assuming only a cofiniteness condition for the vertex operator algebra $V$ and infinite dimensionality of all nonzero $V$-modules (an assumption which is used but not explicitly mentioned in Miyamoto's paper, as pointed out in [M2, [ $\mathrm{AN}]$ ). The main new idea is the use of a generalization of ordinary matrix traces, called "pseudotraces", to construct additional genus-one correlation functions; pseudotraces were successively studied by Arike in [Ar], who obtained a characterization in terms of projective bases (or coordinate systems) for projective modules over associative algebras. In [AM4, Milas and Adamović considered the graded dimensions of characters of modules of certain non-rational vertex operator (super)algebras, and proved the modularity of the differential equations these graded dimensions satisfy.

In this paper, we obtain results that will lead us to a full modular invariance result for logarithmic intertwining operators generalizing the modular invariance in [H2]. We consider a positive energy and $C_{2}$-cofinite vertex operator algebra $V$. We study pseudotraces of products of intertwining operators and genus-one correlation functions as a first step towards this result. Our first concern is on the construction of genus-one correlation functions from products of intertwining operators; to do so, we are naturally led to consider pseudotraces of products of logarithmic intertwining operators. In order for the pseudotrace to be well defined, we consider "logarithmic" modules which admit a right action of some associative algebra, and logarithmic intertwining operators whose products commute with this action. We then develop tools to study these "formal pseudotraces"; in particular, we formulate suitable associativity and commutativity statements for such logarithmic intertwining operator in Theorem 1.32 and Theorem 1.33 .

Using these properties, we verify that many identities for the formal pseudotraces in the semisimple case carry over to the logarithmic setting; however, we see that these pseudotraces satisfy a more complicated system of differential equations than the one in [H2] (Proposition 2.14). Nonetheless it is still possible to prove the convergence of these formal series to multivalued analytic functions (the "genus-one correlation functions") and modular invariance of the space of solutions of the system of differential equations (Proposition 2.24).

In a paper [FH] in preparation jointly with Huang, we will use these results to prove that the space of genus-one correlation functions is invariant under the action of $S L_{2}(\mathbb{Z})$.

\subsection{Summary of results}

In Section 1 we recall concepts from the theory of vertex operator algebras and their modules. We assume that $V=\coprod_{n \in \mathbb{Z}} V_{(n)}$ satisfying $V_{(n)}=0$ for $n \leq 0$ and $V_{(0)}=\mathbb{C} 1$. We will deal with grading-restricted generalized $V$-modules. A grading-restricted generalized $V$-module is a direct sum of the generalized eigenspaces for the operator $L(0)$,

$$
W=\coprod_{n \in \mathbb{C}} W_{[n]}
$$


equipped with a vertex operator map $Y_{W}: V \otimes W \rightarrow W((x))$ satisfying all the axioms for a $V$-module except that for $n \in \mathbb{C}$, the homogeneous subspaces $W_{[n]}$ are the generalized eigenspaces (not necessarily eigenspaces as in the definition of $V$-module) of $L(0)=$ $\operatorname{Res}_{x} x Y W(\omega, x)$ with eigenvalues $n$. A crucial condition for obtaining differential equations for genus-one correlation function is the $C_{2}$-cofiniteness condition, introduced first in [Z]: we shall say that a grading-restricted generalized $V$-module $W$ satisfies the $C_{2}$-cofiniteness condition if the space $C_{2}(W)$ spanned by the set $\left\{v_{-2} w \mid v \in V, w \in W\right\}$ has finite codimension in $W$. We assume that $V$ is $C_{2}$-cofinite. Then all grading-restricted generalized $V$-module is $C_{2}$-cofinite (see [H6]).

In Section 1.3 we recall some notions from the theory of elliptic functions and modular forms: in particular, we recall the Taylor expansion of the Weierstrass elliptic function $\wp$ and its derivatives, and the Fourier ( $q$-expansion) of the Eisenstein series. These expansions, considered as formal power series, will appear as coefficients in identities for the formal $q$-traces and in the system of differential equations for the genus-one correlation function.

We recall the notion of pseudotrace in section 1.4 and define the formal $q$-traces of products of logarithmic intertwining operators; for a fixed vertex operator algebra $V$ and some associative algebra $P$, we consider $V$-modules $\tilde{W}_{i}, i=1, \ldots, n$ equipped with a right action of the algebra $P$ such that $\tilde{W}_{n}$ is projective as right $P$-module; if $\mathcal{Y}_{i}$ are logarithmic intertwining operators of type $\left(\begin{array}{c}\tilde{W}_{i-1} \\ W_{i} \tilde{W}_{i}\end{array}\right), i=1, \ldots, n$, (where we take $\left.\tilde{W}_{0}=\tilde{W}_{n}\right)$ such that for all $i=1, \ldots, n, w_{i} \in W_{i}, \tilde{w}_{i} \in \tilde{W}_{i}$ and $p \in P$,

$$
\mathcal{Y}_{i}(w, x)(\tilde{w} p)=\left(\mathcal{Y}_{i}(w, x) \tilde{w}\right) p,
$$

(we will call logarithmic intertwining operators which satisfy this property $P$ - intertwining operators) then the product $\mathcal{Y}_{1}\left(w_{1}, x_{1}\right) \ldots \mathcal{Y}_{n}\left(w_{n}, x_{n}\right)$ is an element of

$$
\operatorname{End}_{P}\left(\tilde{W}_{n}\right)\left[x_{1}, \ldots, x_{n}\right]\left\{\log x_{1}, \ldots, \log x_{n}\right\}
$$

and it is thus possible to evaluate the pseudotrace

$$
\operatorname{tr}_{\tilde{W}_{n}}^{\phi} \mathcal{Y}_{1}\left(w_{1}, x_{1}\right) \ldots \mathcal{Y}_{n}\left(w_{n}, x_{n}\right) q^{L(0)}
$$

In particular we will consider a map $\mathcal{U}(1): W \rightarrow W[x]$ and study properties of formal $q$-traces obtained by taking pseudotraces of products of geometrically modified logarithmic intertwining operators ([H2])

$$
\mathcal{Y}(\mathcal{U}(x) w, x)
$$

the first goal is to prove absolute convergence of such $q$-traces.

In Section 1.5 we derive identities for the formal $q$-traces; these identities have the same shape as the ones found in [H2], and the main tools used in this section are associativity and commutativity of intertwining operators. It is therefore necessary to obtain a formulation of these duality properties to use in the present context. In Section 1.6, under the assumptions used in [HLZ7, we state and prove suitable commutativity and associativity properties for $P$ intertwining operators; we recall the required background from the theory of tensor product for modules of vertex operator algebras ([HLZ1]-[HLZ8]) in Section 3.2. 
In Section 4 we use the identities obtained in Section 1.5 to obtain differential equation for the formal $q$-traces. We obtain a system of differential equations for which the series

$$
\operatorname{tr}_{\tilde{W}_{n}}^{\phi} \mathcal{Y}_{1}\left(\mathcal{U}\left(q_{z_{1}}\right) L(0)_{n}^{i_{1}} w_{1}, q_{z_{1}}\right) \cdot \ldots \cdot \mathcal{Y}_{n}\left(\mathcal{U}\left(q_{z_{n}}\right) L(0)_{n}^{i_{n}} w_{n}, q_{z_{n}}\right) q^{L(0)-\frac{c}{24}}
$$

$i_{j} \in \mathbb{N}, j=1, \ldots, n$ are solutions (here $L(0)_{n}$ denotes the locally nilpotent part of the operator $L(0))$. Due to the nonsemisimplicity of the operator $L(0)$, the system is not decoupled, but nonetheless the singular points in the variable $q$ are regular, and thus one can prove absolute convergence of the formal $q$-traces in a suitable domain. We then prove modular invariance for the solutions of the system of differential equations in Section 2.2. We consider a space of vector valued functions in the variables $z_{1}, \ldots, z_{n}$ and $\tau$ and we denote the components of these vector valued functions by $\phi_{i_{1}, \ldots, i_{n}}\left(z_{1}, \ldots, z_{n} ; \tau\right)$ for $i_{j} \in \mathbb{N}, j=1, \ldots, n$. The solutions of the system of differential equations are naturally elements of this space. Then, for $g \in S L_{2}(\mathbb{Z})$,

$$
g=\left(\begin{array}{ll}
\alpha & \beta \\
\gamma & \delta
\end{array}\right)
$$

we define the action of $g$ on $\phi_{i_{1}, \ldots, i_{n}}$ by

$$
\begin{aligned}
& \left(g \phi_{i_{1}, \ldots, i_{n}}\right)\left(z_{1}, \ldots, z_{n} ; \tau\right) \\
& \quad=\left(\frac{1}{\gamma \tau+\delta}\right)^{a} \sum_{j_{1}=0}^{\infty} \ldots \sum_{j_{n}=0}^{\infty} \frac{\prod_{k=1}^{n}(\log (\gamma \tau+\delta))^{j_{k}}}{j_{1} ! \cdots j_{n} !} \phi_{i_{1}+j_{1}, \ldots, i_{n}+j_{n}}\left(z_{1}^{\prime}, \ldots, z_{n}^{\prime} ; \tau^{\prime}\right)
\end{aligned}
$$

with $z^{\prime}=\frac{z}{\gamma \tau+\delta}$ and $\tau^{\prime}=\frac{\alpha \tau+\beta}{\gamma \tau+\delta}$. We then prove that the space of solutions of our differential equations is invariant under this action.

Aknowledgements I am grateful to Prof. Yi-Zhi Huang and Prof. James Lepowsky for discussions and suggestions for improvements.

\section{Logarithmic intertwining operators}

\subsection{Generalized modules and logarithmic intertwining operators}

After recalling some notions from logarithmic formal calculus, we recall the definitions of vertex operator algebra and grading-restricted generalized module. We will consider logarithmic intertwining operators for this class of modules. For more details, see [FLM], [FHL], [LL], [H6].

We will denote by $x, y, q, \log x, \log y, \log q, x_{1}, x_{2}, x_{3} \ldots, \log x_{1}, \log x_{2}, \ldots$ independent commuting formal variables. For any set of commuting independent formal variables $X$ and for any vector space $\mathcal{W}$ which does not involve any element of $X$, we denote by $\mathcal{W}\{X\}$ the space of formal series in arbitrary complex powers of the formal variables in $X$. In particular we will consider the space $\mathcal{W}\{x, \log x\}$ : an arbitrary element in this space can be written as

$$
\sum_{m, n \in \mathbb{C}} w_{n, m} x^{n}(\log x)^{m}, \quad w_{n, m} \in \mathcal{W}
$$


The symbol $\frac{d}{d x}$ denotes the linear map (formal differentiation) defined on $W\{x, \log x\}$ by

$$
\begin{aligned}
& \frac{d}{d x}\left(\sum_{m, n \in \mathbb{C}} w_{n, m} x^{n}(\log x)^{m}\right)=\sum_{m, n \in \mathbb{C}}\left((n+1) w_{n+1, m}+(m+1) w_{n+1, m+1}\right) x^{n}(\log x)^{m} \\
&\left(=\sum_{m, n \in \mathbb{C}} n w_{n, m} x^{n-1}(\log x)^{m}+\sum_{m, n \in \mathbb{C}} m w_{n, m} x^{n-1}(\log x)^{m-1}\right) .
\end{aligned}
$$

We will make use of the notation

$$
\begin{aligned}
\log (1-T) & =-\sum_{n=1}^{\infty} \frac{T^{n}}{n} \\
e^{T} & =\sum_{n=0}^{\infty} \frac{T^{n}}{n !}
\end{aligned}
$$

for any $T$ for which these expressions make sense. Also, for commuting independent formal variables $x, y$, we let

$$
\log (x+y)=\log x+\log \left(1+\frac{y}{x}\right)=\log x-\sum_{n=1}^{\infty} \frac{(-1)^{n}}{n}\left(\frac{y}{x}\right)^{n} .
$$

For any formal series in $W\{x, \log x\}$ the following result holds: For $f(x)$ as in (1.1), we have

$$
e^{y \frac{d}{d x}} f(x)=f(x+y)
$$

("Taylor's theorem" for logarithmic formal series) and

$$
e^{y x \frac{d}{d x}} f(x)=f\left(x e^{y}\right) .
$$

In what follows, unless otherwise mentioned, we will fix a vertex operator algebra $V$ of cenrtal charge $c$ such that $V_{(n)}=0$ whenever $n<0$ and $V_{(0)}=\mathbb{C} 1$. We set

$$
C_{2}(V)=\operatorname{span}\left\{v_{-2} u \mid v \in \coprod_{n>0} V_{(n)}, u \in V\right\}
$$

Definition 1.1. We say that $V$ is $C_{2}$-cofinite if

$$
\operatorname{dim} V / C_{2}(V)<\infty
$$

Definition 1.2. A $\mathbb{C}$-graded vector space $W=\coprod_{n \in \mathbb{C}} W_{[n]}$ equipped with a linear map

$$
\begin{aligned}
Y_{W}: V \otimes W & \rightarrow W((x)) \\
v \otimes w & \mapsto Y_{W}(v, x) w
\end{aligned}
$$


is called a grading-restricted generalized $V$-module if all the axioms for $V$-modules are satisfied except that for $n \in \mathbb{C}$, the homogeneous subspaces $W_{[n]}$ are the generalized eigenspaces of $L(0)=\operatorname{Res}_{x} x Y_{W}(\omega, x)$ with eigenvalues $n$, that is, for $n \in \mathbb{C}, w \in W_{[n]}$, there exists $K \in \mathbb{Z}_{+}$, depending on $w$, such that $(L(0)-n)^{K} w=0$. For $w \in W_{[n]}$, we denote the generalized eigenvalue $n$ by wt $w$.

We define homomorphisms (or module maps) and isomorphisms between generalized $V$ modules, generalized $V$-submodules, and quotient generalized $V$-modules in the obvious ways.

For a grading-restricted generalized module $W$, the formal completion of $W$ is the space

$$
\bar{W}=\prod_{n \in \mathbb{C}} W_{[n]}
$$

and for $n \in \mathbb{C}$ we denote the projection from $\bar{W}$ to $W_{[n]}$ by $\pi_{n}$.

Definition 1.3. Let $\left(W_{1}, Y_{1}\right),\left(W_{2}, Y_{2}\right)$ and $\left(W_{3}, Y_{3}\right)$ be grading-restricted generalized modules for a vertex operator algebra $V$. A logarithmic intertwining operator of type $\left(\begin{array}{c}W_{3} \\ W_{1} W_{2}\end{array}\right)$ is a linear map

$$
\mathcal{Y}(\cdot, x) \cdot: W_{1} \otimes W_{2} \rightarrow W_{3}[\log x]\{x\}
$$

or equivalently,

$$
w_{(1)} \otimes w_{(2)} \mapsto \mathcal{Y}\left(w_{(1)}, x\right) w_{(2)}=\sum_{n \in \mathbb{C}} \sum_{k \in \mathbb{N}} w_{(1)_{n ; k}}^{\mathcal{Y}} w_{(2)} x^{-n-1}(\log x)^{k} \in W_{3}[\log x]\{x\}
$$

for all $w_{(1)} \in W_{1}$ and $w_{(2)} \in W_{2}$, such that the following conditions are satisfied: the lower truncation condition: for any $w_{(1)} \in W_{1}, w_{(2)} \in W_{2}$ and $n \in \mathbb{C}$,

$$
w_{(1)}^{\mathcal{Y}}{ }_{n+m ; k} w_{(2)}=0 \quad \text { for } m \in \mathbb{N} \text { sufficiently large, independently of } k \text {; }
$$

the Jacobi identity:

$$
\begin{aligned}
x_{0}^{-1} \delta\left(\frac{x_{1}-x_{2}}{x_{0}}\right) Y_{3}\left(v, x_{1}\right) \mathcal{Y}\left(w_{(1)}, x_{2}\right) w_{(2)} \\
\quad-x_{0}^{-1} \delta\left(\frac{x_{2}-x_{1}}{-x_{0}}\right) \mathcal{Y}\left(w_{(1)}, x_{2}\right) Y_{2}\left(v, x_{1}\right) w_{(2)} \\
=x_{2}^{-1} \delta\left(\frac{x_{1}-x_{0}}{x_{2}}\right) \mathcal{Y}\left(Y_{1}\left(v, x_{0}\right) w_{(1)}, x_{2}\right) w_{(2)}
\end{aligned}
$$

for $v \in V, w_{(1)} \in W_{1}$ and $w_{(2)} \in W_{2}$ (note that the first term on the left-hand side is meaningful because of the lower truncation condition) and the $L(-1)$-derivative property: for any $w_{(1)} \in W_{1}$,

$$
\mathcal{Y}\left(L(-1) w_{(1)}, x\right)=\frac{d}{d x} \mathcal{Y}\left(w_{(1)}, x\right) .
$$

We will denote the space of all logarithmic intertwining operators of type $\left(\begin{array}{c}W_{3} \\ W_{1} W_{2}\end{array}\right)$ by $\mathcal{V}_{W_{1} W_{2}}^{W_{3}}$. 
Note that if the three modules $W_{1}, W_{2}, W_{3}$ are ordinary modules, then all logarithmic intertwining operators are in fact ordinary intertwining operator, i.e., there are no logarithmic terms.

For a grading-restricted generalized $V$-module $W$, we consider the semisimple part of the operator $L(0)$, denoted by $L(0)_{s}$; also denote by $L(0)_{n}$ the locally nilpotent part $L(0)-L(0)_{s}$. Then $L(0)_{n}$ is a module endomorphism of $W$ (i.e., it commutes with the action of $V$ on $W$ ):

Lemma 1.4. Let $\mathcal{Y}(x, w)$ be an intertwining operator; then

$$
L(0)_{n} \mathcal{Y}(x, w)-\mathcal{Y}(x, w) L(0)_{n}=0
$$

Definition 1.5. Let $W$ be a grading-restricted generalized $V$-module for a vertex operator algebra. We define

$$
x^{ \pm L(0)}: W \rightarrow W\{x\}[\log x] \subset W[\log x]\{x\}
$$

by

$$
x^{ \pm L(0)}=x^{ \pm L(0)_{s}} e^{ \pm \log x\left(L(0)-L(0)_{s}\right)}
$$

Lemma 1.6. Using the same notation as above, we have

$$
\frac{d}{d x} x^{L(0)}=L(0) x^{L(0)-1} .
$$

Proposition 1.7. Let $\mathcal{Y}$ be a logarithmic intertwining operator of type $\left(\begin{array}{c}W_{3} \\ W_{1} W_{2}\end{array}\right)$ and let $w \in$ $W_{1}$. Then

$$
e^{y L(-1)} \mathcal{Y}(w, x) e^{-y L(-1)}=\mathcal{Y}\left(e^{y L(-1)} w, x\right)=\mathcal{Y}(w, x+y)
$$

$$
y^{L(0)} \mathcal{Y}(w, x) y^{-L(0)}=\mathcal{Y}\left(y^{L(0)} w, x y\right)
$$

$$
e^{y L(1)} \mathcal{Y}(w, x) e^{-y L(1)}=\mathcal{Y}\left(e^{y(1-y x) L(1)}(1-y x)^{-2 L(0)} w, x(1-y x)^{-1}\right) .
$$

\subsection{Tensor product of modules and associativity of logarithmic intertwining operators}

In this section, we recall the notion of $P(z)$-tensor product for modules of vertex operator algebras introduced in [HL1]-[HL3], [H3] (and then generalized to the logarithmic case in [HLZ1]-[HLZ8 and [H6]), and some related results that we will need later; in particular, we will state associativity for logarithmic intertwining operators.

Let $W_{1}, W_{2}, W_{3}$ be grading-restricted generalized $V$-modules. Related to the concept of logarithmic intertwining operator is that of intertwining map: a $P(z)$-intertwining map of type $\left(\begin{array}{c}W_{3} \\ W_{1} W_{2}\end{array}\right)$ is a linear function

$$
I_{z}: W_{1} \otimes W_{2} \rightarrow \bar{W}_{3}
$$


satisfying the following conditions: the lower truncation condition: for $w_{(1)} \in W_{1}$ and $w_{(2)} \in$ $W_{2}$ and any $n \in \mathbb{C}$,

$$
\pi_{n-m} I\left(w_{(1)} \otimes w_{(2)}\right)=0 \text { for } m \in \mathbb{N} \text { sufficiently large; }
$$

and the Jacobi identity: for $v \in V, w_{(1)} \in W_{1}$ and $w_{(2)} \in W_{2}$,

$$
\begin{aligned}
x_{0}^{-1} \delta & \left(\frac{x_{1}-z}{x_{0}}\right) Y_{3}\left(v, x_{1}\right) I\left(w_{(1)} \otimes w_{(2)}\right) \\
= & z^{-1} \delta\left(\frac{x_{1}-x_{0}}{z}\right) I\left(Y_{1}\left(v, x_{0}\right) w_{(1)} \otimes w_{(2)}\right) \\
& \quad+x_{0}^{-1} \delta\left(\frac{-z+x_{1}}{x_{0}}\right) I\left(w_{(1)} \otimes Y_{2}\left(v, x_{1}\right) w_{(2)}\right) .
\end{aligned}
$$

Let $\mathcal{Y} \in\left(\begin{array}{c}W_{3} \\ W_{1} W_{2}\end{array}\right)$, and let $p \in \mathbb{Z}$; then the map

$$
\begin{aligned}
I_{\mathcal{Y}, p}: W_{1} \otimes W_{2} & \rightarrow W_{3} \\
w_{(1)} \otimes w_{(2)} & \left.\mapsto \mathcal{Y}\left(w_{(1)}, x\right) w_{(2)}\right|_{x^{n}=e^{n(\log z+2 \pi i p)},(\log (x))^{m}=(\log z+2 \pi i p)^{m}}
\end{aligned}
$$

is a well defined $P(z)$-intertwining map of type $\left(\begin{array}{c}W_{3} \\ W_{1} W_{2}\end{array}\right)$; for any $p \in \mathbb{Z}$, the correspondence $\mathcal{Y} \mapsto I_{\mathcal{Y}, p}$ is a bijection with inverse denoted by $I \mapsto \mathcal{Y}_{I, p}([$ HLZ3] $)$.

Definition 1.8 ([HLZ1]-[HLZ7]). Given two grading-restricted generalized $V$-modules $W_{1}$, $W_{2}$, their $P(z)$-tensor product is a third such module, denoted by $W_{1} \otimes_{P(z)} W_{2}$, equipped with a $P(z)$-intertwining map

$$
\bigotimes_{P(z)}: W_{1} \otimes W_{2} \rightarrow \overline{W_{1} \bigotimes_{P(z)} W_{2}}
$$

such that for any grading-restricted generalized $V$-module $W_{3}$ and intertwining map $I$ : $W_{1} \otimes W_{2} \rightarrow \bar{W}_{3}$, there exists a unique $V$-module morphism $\eta: W_{1} \otimes_{P(z)} W_{2} \rightarrow W_{3}$ such that

$$
I=\bar{\eta} \circ \bigotimes_{P(z)},
$$

where $\bar{\eta}$ is the unique map $\bar{\eta}: \overline{W_{1} \bigotimes_{P(z)} W_{2}} \rightarrow \bar{W}_{3}$ extending $\eta$.

From the definition, one can see that given two $V$-modules, if their $P(z)$-tensor product exists then it is unique; moreover we have the following:

Proposition 1.9. Let $W_{1}, W_{2}, W_{3}$ and $W_{4}$ be generalized $V$-modules and $\varphi: W_{1} \rightarrow W_{3}$ and $\psi: W_{2} \rightarrow W_{4}$ be $V$-module homomorphisms. Suppose that the $P(z)$-tensor products $W_{1} \bigotimes_{P(z)} W_{2}$ and $W_{3} \bigotimes_{P(z)} W_{4}$ exist and denote their intertwining maps by $I_{1}, I_{2}$ respectively. Then there exists a unique $V$ homomorphism

$$
\varphi \bigotimes_{P(z)} \psi: W_{1} \bigotimes_{P(z)} W_{2} \rightarrow W_{3} \bigotimes_{P(z)} W_{4}
$$

such that for all $w_{(1)} \in W_{1}$ and $w_{(2)} \in W_{2}$,

$$
I_{2}\left(\varphi\left(w_{(1)}\right) \otimes \psi\left(w_{(2)}\right)\right)=\overline{\varphi \bigotimes_{P(z)} \psi} \circ I_{1}\left(w_{(1)} \otimes w_{(2)}\right) .
$$


We recall some results from [H6] and [HLZ5] concerning the existence of $P(z)$-tensor product and associativity of logarithmic intertwining operators.

Theorem 1.10 ([HLZ5]). Let $V$ be a vertex operator algebra whose category of gradingrestricted generalized modules is closed under $P(z)$-tensor product, and such that every grading-restricted generalized module satisfies the $C_{1}$-cofiniteness condition and the quasifinite dimensionality condition; let $W_{1}, W_{2}, W_{3}, W_{4}, M$ be grading-restricted generalized $V$-modules.

1. Consider intertwining operators $\mathcal{Y}_{1} \in\left(\begin{array}{c}W_{4} \\ W_{1} M\end{array}\right), \mathcal{Y}_{2} \in\left(\begin{array}{c}M \\ W_{2} W_{3}\end{array}\right)$. Then there exists a unique intertwining operator $\mathcal{Y}^{1} \in\left(\begin{array}{c}W_{4} \\ W_{1} \bigotimes_{P\left(z_{0}\right)} W_{2} W_{3}\end{array}\right)$ such that

$$
\begin{aligned}
& \left.\left\langle w_{(4)}^{\prime}, \mathcal{Y}_{1}\left(w_{(1)}, x_{1}\right) \mathcal{Y}_{2}\left(w_{(2)}, x_{2}\right) w_{(3)}\right\rangle\right|_{x_{i}^{n}=e^{n \log z_{i}},\left(\log x_{i}\right)^{m}=\left(\log z_{i}\right)^{m}, i=1,2} \\
& =\left.\left\langle w_{(4)}^{\prime}, \mathcal{Y}^{1}\left(\mathcal{Y}_{\bigotimes_{P\left(z_{0}\right), 0}}\left(w_{(1)}, x_{0}\right) w_{(2)}, x_{2}\right) w_{(3)}\right\rangle\right|_{x_{i}^{n}=e^{n \log z_{i}},\left(\log x_{i}\right)^{m}=\left(\log z_{i}\right)^{m}, i=0,2}
\end{aligned}
$$

whenever $z_{0}=z_{1}-z_{2}$ and $\left|z_{1}\right|>\left|z_{2}\right|>\left|z_{0}\right|>0$, for all $w_{(1)} \in W_{1}, w_{(2)} \in W_{2}, w_{(3)} \in W_{3}$ and $w_{(4)}^{\prime} \in W_{4}^{\prime}$.

2. Let $\mathcal{Y}^{1} \in\left(\begin{array}{c}W_{4} \\ M W_{3}\end{array}\right), \mathcal{Y}^{2} \in\left(\begin{array}{c}M \\ W_{1} W_{2}\end{array}\right)$. Then there exists a unique intertwining operator $\mathcal{Y}_{1}$ of type $\left(\begin{array}{c}W_{4} \\ W_{1} W_{2} \unrhd_{P\left(z_{2}\right)} W_{3}\end{array}\right)$ such that

$$
\begin{aligned}
&\left\langle w_{(4)}^{\prime},\right.\left.\mathcal{Y}^{1}\left(\mathcal{Y}^{2}\left(w_{(1)}, x_{0}\right) w_{(2)}, x_{2}\right) w_{(3)}\right\rangle\left.\right|_{x_{i}^{n}=e^{n \log z_{i},\left(\log x_{i}\right)^{m}=\left(\log z_{i}\right)^{m}, i=0,2}} \\
& \quad=\left.\left\langle w_{(4)}^{\prime}, \mathcal{Y}_{1}\left(w_{(1)}, x_{1}\right) \mathcal{Y}_{\bigotimes_{P\left(z_{2}\right), 0}}\left(w_{(2)}, x_{2}\right) w_{(3)}\right\rangle\right|_{x_{i}^{n}=e^{n \log z_{i}},\left(\log x_{i}\right)^{m}=\left(\log z_{i}\right)^{m}, i=1,2}
\end{aligned}
$$

whenever $\left|z_{1}\right|>\left|z_{2}\right|>\left|z_{1}-z_{2}\right|>0$ for all $w_{(1)} \in W_{1}, w_{(2)} \in W_{2}, w_{(3)} \in W_{3}$ and $w_{(4)}^{\prime} \in W_{4}^{\prime}$.

Theorem 1.11 ([H6]). Assume that $V$ is $C_{2}$-cofinite. Then the category of grading-restricted $V$-modules is closed under $P(z)$-tensor product and every grading-restricted $V$-module is $C_{2}$ cofinite.

\subsection{Elliptic functions and Eisenstein series}

In this section we recall some basic properties of the Weierstrass $\wp$ function and Eisenstein series; in particular, we will be using the Taylor and Fourier $q$-expansions of such functions. For additional background, see $\left[\mathrm{L},[\mathrm{Z}]\right.$. For $z \in \mathbb{C}$, we will use the notation $q_{z}=e^{2 \pi i z}$. We first introduce a formal power series related to the expansion of the Weierstrass $\wp$ function: for $m \geq 0$,

$$
P_{m+1}(x ; q)=(2 \pi i)^{m+1} \sum_{l>0}\left(\frac{l^{m}}{m !} \frac{x^{l}}{1-q^{l}}-\frac{(-1)^{m} l^{m}}{m !} \frac{q^{l} x^{-l}}{1-q^{l}}\right)
$$


where $\left(1-q^{l}\right)^{-1}$ is the power series $\sum_{k>0} q^{l k}$ in the formal variable $q$. For $\tau, z \in \mathbb{C}$ satisfying $\left|q_{\tau}\right|<\left|q_{z}\right|<1$, the series $P_{m+1}\left(q_{z} ; q_{\tau}\right)$ is absolutely convergent, and for $\left|q_{z}\right|<1$ the $q^{-}$ coefficients of $P_{m+1}\left(q_{z} ; q\right)$ are absolutely convergent. Let

$$
\begin{aligned}
& \wp_{1}(z ; \tau)=\frac{1}{z}+\sum_{(k, l) \neq(0,0)}\left(\frac{1}{z-(k \tau+l)}+\frac{1}{k \tau+l}+\frac{z}{(k \tau+l)^{2}}\right) \\
& \wp_{2}(z ; \tau)=\frac{1}{z^{2}}+\sum_{(k, l) \neq(0,0)}\left(\frac{1}{(z-(k \tau+l))^{2}}-\frac{1}{(k \tau+l)^{2}}\right) ;
\end{aligned}
$$

and for $m \geq 2$, let

$$
\wp_{m+1}(z ; \tau)=-\frac{1}{m} \frac{\partial}{\partial z} \wp_{m}(z ; \tau) .
$$

These functions have Laurent expansion

$$
\wp_{m}(z ; \tau)=\frac{1}{z^{m}}+(-1)^{m} \sum_{k \geq 1}\left(\begin{array}{c}
2 k+1 \\
m-1
\end{array}\right) G_{2 k+2}(\tau) z^{2 k+2-m}
$$

in the region $0<|z|<\min (1,|\tau|)$, where $G_{2 k+2}(\tau)$ are the Eisenstein series defined by

$$
G_{2 k+2}(\tau)=\sum_{(m, l) \in \mathbb{Z}^{2} \backslash(0,0)} \frac{1}{(m \tau+l)^{2 k+2}}
$$

for $k \geq 1$. Moreover, let

$$
G_{2}(\tau)=\frac{\pi^{2}}{3}+\sum_{m \in \mathbb{Z} \backslash\{0\}} \sum_{l \in \mathbb{Z}} \frac{1}{(m \tau+l)^{2}} .
$$

It is known that the Eisenstein series have $q$-expansion

$$
G_{2 k+2}(\tau)=2 \zeta(2 k+2)+\frac{2(2 \pi i)^{2 k+2}}{(2 k+1) !} \sum_{l=1}^{\infty} \frac{l^{2 k+1} q_{\tau}^{l}}{1-q_{\tau}^{l}}
$$

We use the following notation to denote these as formal power series in the variable $q$ :

$$
\tilde{G}_{2 k+2}(q)=2 \zeta(2 k+2)+\frac{2(2 \pi i)^{2 k+2}}{(2 k+1) !} \sum_{l=1}^{\infty} \frac{l^{2 k+1} q^{l}}{1-q^{l}}, \quad k \in \mathbb{N}
$$

and similarly for the expansion of the elliptic functions

$$
\tilde{\wp}_{m}(x ; q)=\frac{1}{x^{m}}+(-1)^{m} \sum_{k=1}^{\infty}\left(\begin{array}{c}
2 k+1 \\
m-1
\end{array}\right) \tilde{G}_{2 k+2}(q) x^{2 k+2-m}, \quad m=1,2, \ldots
$$

For $z \in \mathbb{C}$ such that $0<|z|<1$,

$$
\tilde{\wp}_{m}(z ; q)=(-1)^{m}\left(P_{m}\left(q_{z} ; q\right)-\frac{\partial^{m-1}}{\partial z^{m-1}}\left(\tilde{G}_{2}(q) z-\pi i\right)\right)
$$

and $\tilde{\wp}_{m}\left(z ; q_{\tau}\right)=\wp_{m}(z ; \tau)$. The following is well known: 
Proposition 1.12. For any element $g=\left(\begin{array}{ll}\alpha & \beta \\ \gamma & \delta\end{array}\right) \in S L_{2}(\mathbb{Z})$, and $m=1,2, \ldots$

$$
\left.\wp_{m}\right|_{g}(z ; \tau):=(\gamma \tau+\delta)^{-m} \wp_{m}\left(\frac{z}{\gamma \tau+\delta} ; \frac{\alpha \tau+\beta}{\gamma \tau+\delta}\right)=\wp_{m}(z ; \tau)
$$

if $m>1$,

$$
\wp_{m}(z+\tau ; \tau)=\wp_{m}(z+1 ; \tau)=\wp_{m}(z ; \tau)
$$

and

$$
\begin{gathered}
\wp_{1}(z+1 ; \tau)=\wp_{1}(z ; \tau)+G_{2}(q) \\
\wp_{1}(z+\tau ; \tau)=\wp_{1}(z ; \tau)+G_{2}(\tau) \tau-2 \pi i
\end{gathered}
$$

Proposition 1.13. Let $f(q)$ be a modular form of weight $k$. Then the function $\vartheta_{k}(f)$ defined by

$$
(2 \pi i)^{2} q \frac{d}{d q} f(q)+k G_{2}(q) f(q)
$$

is a modular form of weight $k+2$.

\subsection{Pseudotraces}

Let $P$ be a finite-dimensional associative algebra over $\mathbb{C}$. We will say that a linear function $\phi: P \rightarrow \mathbb{C}$ is symmetric if $\phi(p q)=\phi(q p)$ for all $p, q \in P$, and denote by $S L F(P)$ the vector space of all such functions; $S L F(P) \simeq(P /[P, P])^{*}$.

Let $M$ be a finitely generated projective right $P$-module. It is well known that this condition is equivalent to the existence of a projective basis for $M$, that is a pair of sets $\left\{m_{i}\right\}_{i=1}^{n} \subseteq M,\left\{\alpha_{i}\right\}_{i=1}^{n} \subseteq \operatorname{Hom}_{P}(M, P)$ such that for all $m \in M$,

$$
m=\sum_{i=1}^{n} m_{i} \alpha_{i}(m)
$$

Definition 1.14. Let $\phi \in S L F(P)$. The pseudtrace map $\phi_{M}$ on $\operatorname{End}_{P}(M)$ associated to $\phi$ is the function $\phi_{M}$ defined by

$$
\phi_{M}(\alpha)=\phi\left(\sum_{i=1}^{n} \alpha_{i}\left(\alpha\left(m_{i}\right)\right)\right)
$$

for all $\alpha \in \operatorname{End}_{P}(M)$.

It is easy to show that the definition of pseudotrace does not depend on the choice of projective basis for $M$. Pseudotrace maps are an extension of regular trace functions, and share similar properties; it is easy to see that the pseudotrace of a product is invariant under cyclic permutation of the factors [AN]: 
Proposition 1.15. Let $M_{1}$ and $M_{2}$ be two right projective P-modules, and consider homomorphisms $\alpha \in \operatorname{Hom}_{P}\left(M_{1}, M_{2}\right)$ and $\beta \in \operatorname{Hom}_{P}\left(M_{2}, M_{1}\right)$; then

$$
\phi_{M_{1}}(\beta \circ \alpha)=\phi_{M_{2}}(\alpha \circ \beta) .
$$

Importantly, for a given associative algebra $P$, the pseudotraces of representations of $P$ span the space $S L F(P)$ of symmetric linear functions on $P$ (see for example [Ar, Miy2).

\subsection{Formal $q$-traces of logarithmic intertwining operators}

In this section we consider grading-restricted $V$-modules which admit a right action (by module endomorphisms) of a finite-dimensional associative algebra $P$. We then consider products of intertwining operators which commute with this action; in particular, we are able to define the formal $q$-trace of such products by using pseudotraces on the $P$-endomorphism ring of the $L(0)$ generalized eigenspaces in the grading-restricted generalized $V$-modules. We use properties of pseudotraces and intertwining operators to derive identities for the formal $q$-traces.

Let $P$ be a finite-dimensional associative algebra equipped with a symmetric linear function $\phi$. We say that a grading-restricted generalized $V$-module $W$ is a $V$ - $P$-bimodule if $W$ is a right $P$ module and $P$ acts on $W$ by $V$-module endomorphisms, that is, for any $v \in V$, $w \in W$, and $p \in P$,

$$
Y(v, x)(w p)=(Y(v, x) w) p .
$$

Proposition 1.16. Let $W_{[n]}$ be the $L(0)$-generalized eigenspace of $W$ for the eigenvalue $n$. Then $W_{[n]}$ is a $P$-submodule of $W$; if $W$ is a projective right $P$-module, so is $W_{[n]}$.

Proof. This is clear since the action of $P$ commutes with $L(0)$ : let $p \in P$ and $w \in W_{[n]}$; then there exists $k \in \mathbb{N}$ such that $(L(0)-n)^{k} w=0$. Then

$$
(L(0)-n)^{k}(w p)=\left((L(0)-n)^{k} w\right) p=0
$$

which proves the first part of the claim; the second part follows since $W_{[n]}$ is a direct summand of $W$.

As a consequence, the action of $P$ commutes with $L(0)_{s}$ and $L(0)_{n}$. Suppose now $W$ is a grading-restricted generalized $V$-module, projective as right $P$-module. Then for any generalized eigenspace $W_{[n]}$, we have the pseudotrace

$$
\phi_{W_{[n]}}: \operatorname{End}_{P}\left(W_{[n]}\right) \rightarrow \mathbb{C} ;
$$

and for a given element $a\left(x_{1}, \ldots, x_{k}\right) \in \operatorname{End}_{P}(W)\left\{x_{1}, \ldots, x_{k}, \log x_{1}, \ldots, \log x_{k}\right\}$, we define

$$
\operatorname{tr}_{W}^{\phi} a\left(x_{1}, \ldots, x_{n}\right) q^{L(0)}=\left.\sum_{n \in \mathbb{C}} \phi_{W_{[n]}}\left(\pi_{n} a\left(x_{1}, \ldots, x_{k}\right) \sum_{i=0}^{\infty} \frac{\left(L(0)_{n}\right)^{i}}{i !}(\log q)^{i}\right)\right|_{W_{[n]}} q^{n}
$$

where $\pi_{n}: W \rightarrow W_{[n]}$ is the projection on the generalized eigenspace $W_{[n]}$. Note that since $L(0)_{n}$ is locally nilpotent, the summation over $i$ is finite for any value of $n \in \mathbb{C}$. If $W$ has finite length $l$, then the powers of $\log q$ are globally bounded by $l$. 
Remark 1.17. Suppose $T \in \operatorname{End}_{P}\left(W_{[n]}\right)$, and let $\left\{w_{i}\right\}_{i=1}^{s},\left\{\alpha_{i}\right\}_{i=1}^{s}$ be a projective basis for $W_{[n]}$. Let $w_{i}^{\prime} \in W_{[n]}^{*}$ be the linear function defined by $\left\langle w_{i}^{\prime}, w\right\rangle=\phi\left(\alpha_{i}(w)\right)$ for all $w \in W_{[n]}$. Then one can express the pseudotrace of $T$ as

$$
\phi_{W_{[n]}}(T)=\sum_{i=1}^{s}\left\langle w_{i}^{\prime}, T w_{i}\right\rangle .
$$

In particular, for any $n \in \mathbb{C}$ let $\left\{w_{n, i}\right\}_{i=1}^{s_{n}},\left\{\alpha_{n, i}\right\}_{i=1}^{s_{n}}$ be a projective basis of $W_{[n]}$ and let $a\left(x_{1}, \ldots, x_{k}\right)$ as above, one can express the $q$-trace of $a\left(x_{1}, \ldots, x_{n}\right)$ as

$$
\operatorname{tr}_{W}^{\phi} a\left(x_{1}, \ldots, x_{n}\right) q^{L(0)}=\sum_{n \in \mathbb{C}} \sum_{i=1}^{s_{n}}\left(\sum_{j=1}^{\infty}\left\langle w_{n, i}^{\prime}, a\left(x_{1}, \ldots, a_{n}\right) \frac{\left(L(0)_{n}\right)^{j}}{j !} w_{n, i}\right\rangle(\log q)^{j}\right) q^{n}
$$

where $w_{n, i}^{\prime}$ is defined as above and extended to an element of $W^{\prime}$ by letting it map to 0 the generalized subspaces for eigenvalues different from $n$.

Now let $W_{i}, \tilde{W}_{i}, i=1, \ldots, n$, be grading-restricted generalized modules for $V$, and suppose $\tilde{W}_{n}$ is a $V$-P-bimodules, projective as right $P$-module. Moreover, consider logarithmic intertwining operators $\mathcal{Y}_{i} \in\left(\begin{array}{l}\tilde{W}_{i-1} \\ W_{i} \tilde{W}_{i}\end{array}\right), i=1, \ldots, n$, where we use the convention $\tilde{W}_{0}=\tilde{W}_{n}$. If the action of $P$ commutes with the product of these intertwining operators, i.e., for all $w_{i} \in W_{i}, \tilde{w}_{n} \in \tilde{W}_{n}$, and $p$ in $P$,

$$
\mathcal{Y}_{1}\left(w_{1}, x_{1}\right) \cdots \mathcal{Y}_{n}\left(w_{n}, x_{n}\right)\left(\tilde{w}_{n} p\right)=\left(\mathcal{Y}_{1}\left(w_{1}, x_{1}\right) \cdots \mathcal{Y}_{n}\left(w_{n}, x_{n}\right) \tilde{w}_{n}\right) p
$$

then we have the formal q-trace

$$
\operatorname{tr}_{\tilde{W}_{n}}^{\phi} \mathcal{Y}_{1}\left(w_{1}, x_{1}\right) \cdots \mathcal{Y}_{n}\left(w_{n}, x_{n}\right) q^{L(0)}
$$

Remark 1.18. Using the same notation as above, suppose $\tilde{W}_{i}$ for $i=1, \ldots, n$ are $V$ - $P$ bimodules, with $\tilde{W}_{n}$ projective as right $P$-module, and the action of $P$ commutes with all the intertwining operators, i.e., for all $i=1, \ldots, n$ for all $w_{i} \in W_{i}, \tilde{w}_{i} \in \tilde{W}_{i}$, and $p$ in $P$,

$$
\mathcal{Y}\left(w_{i}, x\right)\left(\tilde{w}_{i} p\right)=\left(\mathcal{Y}\left(w_{i}, x\right) \tilde{w}_{i}\right) p .
$$

Then the product of the intertwining operators commute with the action of $P$ and

$$
\operatorname{tr}_{\tilde{W}_{n}}^{\phi} \mathcal{Y}_{1}\left(w_{1}, x_{1}\right) \cdots \mathcal{Y}_{n}\left(w_{n}, x_{n}\right) q^{L(0)}
$$

is well defined.

Definition 1.19. Let $W$ be a grading-restricted generalized $V$-module and $W_{1}, W_{2}$ be $V$ $P$-bimodules; we will say that a logarithmic intertwining operator $\mathcal{Y}$ of type $\left(\begin{array}{c}W_{2} \\ W W_{1}\end{array}\right)$ is a $P$-intertwining operator if

$$
\mathcal{Y}(w, x)\left(w_{1} p\right)=\left(\mathcal{Y}(w, x) w_{1}\right) p
$$

for all $p \in P, w \in W, W_{1} \in W_{1}$. 
Remark 1.20. Note that for any set of intertwining operators $\mathcal{Y}_{1}, \ldots, \mathcal{Y}_{n}$ of the above types, one can always consider $\tilde{W}_{i}$ as a projective right $P$-module with $P=\mathbb{C}$ and $\phi=1_{\mathbb{C}}$. In this case, $\operatorname{tr}^{\phi}$ corresponds to the ordinary matrix trace of the action of the intertwining operators on the vector space $\tilde{W}_{n}$.

Remark 1.21. Using the same notation as in Remark 1.17, we can express the formal $q$-trace as

$$
\begin{aligned}
& \operatorname{tr}_{\tilde{W}_{n}}^{\phi} \mathcal{Y}_{1}\left(w_{1}, x_{1}\right) \cdots \mathcal{Y}_{n}\left(w_{n}, x_{n}\right) q^{L(0)} \\
& \quad=\sum_{n \in \mathbb{C}} \sum_{i=1}^{s_{n}}\left(\sum_{j=1}^{\infty}\left\langle w_{n, i}^{\prime}, \mathcal{Y}_{1}\left(w_{1}, x_{1}\right) \cdots \mathcal{Y}_{n}\left(w_{n}, x_{n}\right) \frac{\left(L(0)_{n}\right)^{j}}{j !} w_{n, i}\right\rangle(\log q)^{j}\right) q^{n} .
\end{aligned}
$$

In particular, as a formal series in the variables $q, \log q$, its coefficients are finite sums of genus-zero correlation functions; one will be able to use properties of these correlation functions to obtain properties for formal $q$ traces.

Following [H2], we use the concept of geometrically modified intertwining operator. Let $A_{j}, j \in \mathbb{Z}_{+}$be the numbers defined by the formal relation

$$
\frac{1}{2 \pi i} \log (1+2 \pi i y)=\left(\exp \left(\sum_{j \in \mathbb{Z}_{+}} A_{j} y^{j+1} \frac{\partial}{\partial y}\right)\right) y
$$

and let $L_{+}(A)=\sum_{j \in Z_{+}} A_{j} L(j)$; then the operator $\mathcal{U}(1)$ is defined by

$$
\mathcal{U}(1)=(2 \pi i)^{L(0)} e^{-L_{+}(A)} .
$$

Also let $\mathcal{U}(x)=x^{L(0)} \mathcal{U}(1)$, for any formal expression $x$ for which the expression makes sense: it follows that

$$
y^{L(0)} \mathcal{U}(x)=\mathcal{U}(y x) .
$$

Definition 1.22 ([표). Let $W_{1}, W_{2}, W_{3}$ be grading-restriced generalized $V$-modules, and $\mathcal{Y}$ a logarithmic intertwining operator of type $\left(\begin{array}{c}W_{3} \\ W_{1} W_{2}\end{array}\right)$. The operator $\mathcal{Y}(\mathcal{U}(x) w, x)$ is called a geometrically modified logarithmic intertwining operator.

Here we recall some of the properties of the operator $\mathcal{U}(1)$ and the geometrically modified logarithmic intertwining operators; see [H2] for these results (the proofs in [H2] are for ordinary intertwining operators but the proofs for logarithmic intertwining operators are exactly the same).

Lemma 1.23. Let $\mathcal{Y}$ be a logarithmic intertwining operator of type $\left(\begin{array}{c}W_{3} \\ W_{1}, W_{2}\end{array}\right)$ for grading restricted generalized $V$-modules $W_{1}, W_{2}, W_{3}$. Then for $u \in V$ and $w \in W_{1}$,

$$
\begin{aligned}
& {\left[Y\left(\mathcal{U}\left(x_{1}\right) u, x_{1}\right), \mathcal{Y}\left(\mathcal{U}\left(x_{2}\right) w, x_{2}\right)\right]} \\
& \quad=2 \pi i \operatorname{Res}_{y} \delta\left(\frac{x_{1}}{e^{2 \pi i y} x_{2}}\right) \mathcal{Y}\left(\mathcal{U}\left(x_{2}\right) Y(u, y) w, x_{2}\right)
\end{aligned}
$$


Lemma 1.24. Let $W_{1}, W_{2}, W_{3}$ be grading-restricted generalized $V$-modules, and $\mathcal{Y}$ and logarithmic intertwining operator of type $\left(\begin{array}{c}W_{3} \\ W_{1} W_{2}\end{array}\right)$. Then for any $w_{1} \in W_{1}$,

$$
\mathcal{Y}\left(\mathcal{U}(x) L(-1) w_{1}, x\right)=2 \pi i x \frac{d}{d x} \mathcal{Y}\left(\mathcal{U}(x) w_{1}, x\right)
$$

Lemma 1.25. For any grading-restricted generalized $V$-module $W$ and $u \in V$, we have the $\mathcal{U}(x)$ conjugation property

$$
\mathcal{U}(x) Y(u, y)=Y\left(\mathcal{U}\left(x e^{2 \pi i y}\right) u, x\left(e^{2 \pi i y}-1\right)\right) \mathcal{U}(x) .
$$

We will consider formal $q$-traces of products of geometrically modified logarithmic intertwining operators

$$
\operatorname{tr}_{\tilde{W}_{n}}^{\phi} \mathcal{Y}_{1}\left(\mathcal{U}\left(x_{1}\right) w_{1}, x_{1}\right) \cdots \mathcal{Y}_{n}\left(\mathcal{U}\left(x_{n}\right) w_{n}, x_{n}\right) q^{L(0)}
$$

for intertwining operators whose product commutes with the action of $P$. Many of the properties of regular traces which hold in the completely reducible case carry over to the logarithmic setting.

In the following, for any $v \in V$, we denote by $o(v)$ the constant term of the operator $Y\left(x^{L(0)} v, x\right)$ acting on a generalized $V$-module; that is,

$$
o(v)=\operatorname{Res}_{x} x^{-1} Y\left(x^{L(0)} v, x\right)=v_{\mathrm{wt}} v-1 .
$$

Lemma 1.26. Consider grading-restricted generalized $V$-modules $W_{i}, \tilde{W}_{i}$ for $i=1, \ldots, n$, with $\tilde{W}_{0}=\tilde{W}_{n}$, and logarithmic intertwining operators $\mathcal{Y}_{i}$ of type $\left(\begin{array}{l}\tilde{W}_{i-1} \\ W_{i} \tilde{W}_{i}\end{array}\right)$ for $i=1, \ldots, n$. Moreover, suppose $\tilde{W}_{0}$ is a V-P-bimodule projective as a right P-module for some algebra $P$ equipped with a symmetric linear function $\phi$, and that the product of the intertwining operators $\mathcal{Y}_{1}, \ldots, \mathcal{Y}_{n}$ commutes with the action of $P$. Then for any $v \in V, w_{i} \in W_{i}$, we have

$$
\begin{gathered}
\operatorname{tr}_{\tilde{W}_{n}}^{\phi} Y(\mathcal{U}(x) u, x) \mathcal{Y}_{1}\left(\mathcal{U}\left(x_{1}\right) w_{1}, x_{1}\right) \cdots \mathcal{Y}_{n}\left(\mathcal{U}\left(x_{n}\right) w_{n}, x_{n}\right) q^{L(0)} \\
=\sum_{i=1}^{n} \sum_{m \geq 0} P_{m+1}\left(\frac{x_{i}}{x} ; q\right) \operatorname{tr}_{\tilde{W}_{n}}^{\phi} \mathcal{Y}_{1}\left(\mathcal{U}\left(x_{1}\right) w_{1}, x_{1}\right) \cdot \\
\cdots \mathcal{Y}_{i-1}\left(\mathcal{U}\left(x_{i-1}\right) w_{i-1}, x_{i-1}\right) \mathcal{Y}_{i}\left(\mathcal{U}\left(x_{i}\right) u_{m} w_{i}, x_{i}\right) \cdot \\
\cdot \mathcal{Y}_{i+1}\left(\mathcal{U}\left(x_{i+1}\right) w_{i+1}, x_{i+1}\right) \cdots \mathcal{Y}_{n}\left(\mathcal{U}\left(x_{n}\right) w_{n}, x_{n}\right) q^{L(0)} \\
+\operatorname{tr}_{\tilde{W}_{n}}^{\phi} o(\mathcal{U}(1) u) \mathcal{Y}_{1}\left(\mathcal{U}\left(x_{1}\right) w_{1}, x_{1}\right) \cdots \mathcal{Y}_{n}\left(\mathcal{U}\left(x_{n}\right) w_{n}, x_{n}\right) q^{L(0)}
\end{gathered}
$$

and

$$
\begin{aligned}
& \sum_{i=1}^{n} \operatorname{tr}_{\tilde{W}_{n}}^{\phi} \mathcal{Y}_{1}\left(\mathcal{U}\left(x_{1}\right) w_{1}, x_{1}\right) \cdots \mathcal{Y}_{i-1}\left(\mathcal{U}\left(x_{i-1}\right) w_{i-1}, x_{i-1}\right) \\
& \quad \cdot \mathcal{Y}_{i}\left(\mathcal{U}\left(x_{i}\right) u_{0} w_{i}, x_{i}\right) \mathcal{Y}_{i+1}\left(\mathcal{U}\left(x_{i+1}\right) w_{i+1}, x_{i+1}\right) \cdots \mathcal{Y}_{n}\left(\mathcal{U}\left(x_{n}\right) w_{n}, x_{n}\right) q^{L(0)} \\
& \quad=0
\end{aligned}
$$


Proof. By the commutator formula,

$$
\begin{aligned}
Y(\mathcal{U}(x) u, x) \mathcal{Y}_{1}\left(\mathcal{U}\left(x_{1}\right) w_{1}, x_{1}\right) \cdots \mathcal{Y}_{n}\left(\mathcal{U}\left(x_{n}\right) w_{n}, x_{n}\right) \\
=\sum_{i=1}^{n} \mathcal{Y}_{1}\left(\mathcal{U}\left(x_{1}\right) w_{1}, x_{1}\right) \cdots \mathcal{Y}_{i-1}\left(\mathcal{U}\left(x_{i-1}\right) w_{i-1}, x_{i-1}\right) \\
\cdot\left[Y(\mathcal{U}(x) u, x), \mathcal{Y}_{i}\left(\mathcal{U}\left(x_{i}\right) w_{i}, x_{i}\right)\right] \mathcal{Y}_{i+1}\left(\mathcal{U}\left(x_{i+1}\right) w_{i+1}, x_{i+1}\right) \cdot \\
\cdots \mathcal{Y}_{n}\left(\mathcal{U}\left(x_{n}\right) w_{n}, x_{n}\right) \\
+\mathcal{Y}_{1}\left(\mathcal{U}\left(x_{1}\right) w_{1}, x_{1}\right) \cdots \mathcal{Y}_{n}\left(\mathcal{U}\left(x_{n}\right) w_{n}, x_{n}\right) Y(\mathcal{U}(x) u, x) \\
=\sum_{i=1}^{n} 2 \pi i \operatorname{Res}_{y} \delta\left(\frac{x}{e^{2 \pi i y} x_{i}}\right) \mathcal{Y}_{1}\left(\mathcal{U}\left(x_{1}\right) w_{1}, x_{1}\right) \cdots \mathcal{Y}_{i-1}\left(\mathcal{U}\left(x_{i-1}\right) w_{i-1}, x_{i-1}\right) . \\
\cdot \mathcal{Y}_{i}\left(\mathcal{U}\left(x_{1}\right) Y(u, y) w_{i}, x_{i}\right) \mathcal{Y}_{i+1}\left(\mathcal{U}\left(x_{i+1}\right) w_{i+1}, x_{i+1}\right) . \\
\cdots \mathcal{Y}_{n}\left(\mathcal{U}\left(x_{n}\right) w_{n}, x_{n}\right) \\
+\mathcal{Y}_{1}\left(\mathcal{U}\left(x_{1}\right) w_{1}, x_{1}\right) \cdots \mathcal{Y}_{n}\left(\mathcal{U}\left(x_{n}\right) w_{n}, x_{n}\right) Y(\mathcal{U}(x) u, x)
\end{aligned}
$$

Since $P$ acts on $\tilde{W}_{n}$ as $V$-module endomorphisms, and the product of the intertwining operators $\mathcal{Y}_{1} \ldots \mathcal{Y}_{n}$ commutes with $P$, the pseudotrace of each term in this expression is well defined. Therefore, by linearity of pseudotraces, $q^{L(0)}$ conjugation property and cyclic property of pseudotraces,

$$
\begin{aligned}
& \operatorname{tr}_{\tilde{W}_{n}}^{\phi} Y(\mathcal{U}(x) u, x) \mathcal{Y}_{1}\left(\mathcal{U}\left(x_{1}\right) w_{1}, x_{1}\right) \cdots \mathcal{Y}_{n}\left(\mathcal{U}\left(x_{n}\right) w_{n}, x_{n}\right) q^{L(0)} \\
& =\sum_{i=1}^{n} \operatorname{tr}_{\tilde{W}_{n}}^{\phi} 2 \pi i \operatorname{Res}_{y} \delta\left(\frac{x}{e^{2 \pi i y} x_{i}}\right) \mathcal{Y}_{1}\left(\mathcal{U}\left(x_{1}\right) w_{1}, x_{1}\right) \cdots \mathcal{Y}_{i-1}\left(\mathcal{U}\left(x_{i-1}\right) w_{i-1}, x_{i-1}\right) \text {. } \\
& \text { - } \mathcal{Y}_{i}\left(\mathcal{U}\left(x_{1}\right) Y(u, y) w_{i}, x_{i}\right) \mathcal{Y}_{i+1}\left(\mathcal{U}\left(x_{i+1}\right) w_{i+1}, x_{i+1}\right) \text {. } \\
& \cdots \mathcal{Y}_{n}\left(\mathcal{U}\left(x_{n}\right) w_{n}, x_{n}\right) q^{L(0)} \\
& +\operatorname{tr}_{\tilde{W}_{n}}^{\phi} \mathcal{Y}_{1}\left(\mathcal{U}\left(x_{1}\right) w_{1}, x_{1}\right) \cdots \mathcal{Y}_{n}\left(\mathcal{U}\left(x_{n}\right) w_{n}, x_{n}\right) Y(\mathcal{U}(x) u, x) q^{L(0)} \\
& =\sum_{i=1}^{n} \operatorname{tr}_{\tilde{W}_{n}}^{\phi} 2 \pi i \operatorname{Res}_{y} \delta\left(\frac{x}{e^{2 \pi i y} x_{i}}\right) \mathcal{Y}_{1}\left(\mathcal{U}\left(x_{1}\right) w_{1}, x_{1}\right) \cdots \mathcal{Y}_{i-1}\left(\mathcal{U}\left(x_{i-1}\right) w_{i-1}, x_{i-1}\right) \text {. } \\
& \cdot \mathcal{Y}_{i}\left(\mathcal{U}\left(x_{1}\right) Y(u, y) w_{i}, x_{i}\right) \mathcal{Y}_{i+1}\left(\mathcal{U}\left(x_{i+1}\right) w_{i+1}, x_{i+1}\right) \cdot \\
& \cdots \mathcal{Y}_{n}\left(\mathcal{U}\left(x_{n}\right) w_{n}, x_{n}\right) q^{L(0)} \\
& +\operatorname{tr}_{\tilde{W}_{n}}^{\phi} \mathcal{Y}_{1}\left(\mathcal{U}\left(x_{1}\right) w_{1}, x_{1}\right) \cdots \mathcal{Y}_{n}\left(\mathcal{U}\left(x_{n}\right) w_{n}, x_{n}\right) q^{L(0)} Y\left(\mathcal{U}\left(\frac{x}{q}\right) u, \frac{x}{q}\right) \\
& =\sum_{i=1}^{n} \operatorname{tr}_{\tilde{W}_{n}}^{\phi} 2 \pi i \operatorname{Res}_{y} \delta\left(\frac{x}{e^{2 \pi i y} x_{i}}\right) \mathcal{Y}_{1}\left(\mathcal{U}\left(x_{1}\right) w_{1}, x_{1}\right) \cdots \mathcal{Y}_{i-1}\left(\mathcal{U}\left(x_{i-1}\right) w_{i-1}, x_{i-1}\right) \text {. } \\
& \text { - } \mathcal{Y}_{i}\left(\mathcal{U}\left(x_{1}\right) Y(u, y) w_{i}, x_{i}\right) \mathcal{Y}_{i+1}\left(\mathcal{U}\left(x_{i+1}\right) w_{i+1}, x_{i+1}\right) \text {. } \\
& \cdots \mathcal{Y}_{n}\left(\mathcal{U}\left(x_{n}\right) w_{n}, x_{n}\right) q^{L(0)} \\
& +\operatorname{tr}_{\tilde{W}_{n}}^{\phi} Y\left(\mathcal{U}\left(\frac{x}{q}\right) u, \frac{x}{q}\right) \mathcal{Y}_{1}\left(\mathcal{U}\left(x_{1}\right) w_{1}, x_{1}\right) \cdots \mathcal{Y}_{n}\left(\mathcal{U}\left(x_{n}\right) w_{n}, x_{n}\right) q^{L(0)}
\end{aligned}
$$




$$
\begin{aligned}
=\sum_{i=1}^{n} & \operatorname{tr}_{\tilde{W}_{n}}^{\phi} 2 \pi i \operatorname{Res}_{y} \delta\left(\frac{x}{e^{2 \pi i y} x_{i}}\right) \mathcal{Y}_{1}\left(\mathcal{U}\left(x_{1}\right) w_{1}, x_{1}\right) \cdots \mathcal{Y}_{i-1}\left(\mathcal{U}\left(x_{i-1}\right) w_{i-1}, x_{i-1}\right) \\
& \cdot \mathcal{Y}_{i}\left(\mathcal{U}\left(x_{1}\right) Y(u, y) w_{i}, x_{i}\right) \mathcal{Y}_{i+1}\left(\mathcal{U}\left(x_{i+1}\right) w_{i+1}, x_{i+1}\right) \\
& \cdots \mathcal{Y}_{n}\left(\mathcal{U}\left(x_{n}\right) w_{n}, x_{n}\right) q^{L(0)} \\
+ & \left(q^{-x \frac{\partial}{\partial x}}\right) \operatorname{tr}_{\tilde{W}_{n}}^{\phi} Y(\mathcal{U}(x) u, x) \mathcal{Y}_{1}\left(\mathcal{U}\left(x_{1}\right) w_{1}, x_{1}\right) \cdots \mathcal{Y}_{n}\left(\mathcal{U}\left(x_{n}\right) w_{n}, x_{n}\right) q^{L(0)}
\end{aligned}
$$

and thus

$$
\begin{aligned}
& \left(1-q^{-x \frac{\partial}{\partial x}}\right) \operatorname{tr}_{\tilde{W}_{n}}^{\phi} Y(\mathcal{U}(x) u, x) \mathcal{Y}_{1}\left(\mathcal{U}\left(x_{1}\right) w_{1}, x_{1}\right) \cdots \mathcal{Y}_{n}\left(\mathcal{U}\left(x_{n}\right) w_{n}, x_{n}\right) q^{L(0)} \\
& =\sum_{i=1}^{n} \operatorname{tr}_{\tilde{W}_{n}}^{\phi} 2 \pi i \operatorname{Res}_{y} \delta\left(\frac{x}{e^{2 \pi i y} x_{i}}\right) \mathcal{Y}_{1}\left(\mathcal{U}\left(x_{1}\right) w_{1}, x_{1}\right) \cdots \mathcal{Y}_{i-1}\left(\mathcal{U}\left(x_{i-1}\right) w_{i-1}, x_{i-1}\right) \text {. } \\
& \text { - } \mathcal{Y}_{i}\left(\mathcal{U}\left(x_{1}\right) Y(u, y) w_{i}, x_{i}\right) \mathcal{Y}_{i+1}\left(\mathcal{U}\left(x_{i+1}\right) w_{i+1}, x_{i+1}\right) \cdot \\
& \cdots \mathcal{Y}_{n}\left(\mathcal{U}\left(x_{n}\right) w_{n}, x_{n}\right) q^{L(0)} \\
& =\sum_{i=1}^{n} \operatorname{tr}_{\tilde{W}_{n}}^{\phi} 2 \pi i \operatorname{Res}_{y} e^{2 \pi i y x_{i} \frac{\partial}{\partial x_{i}}} \delta\left(\frac{x}{x_{i}}\right) \mathcal{Y}_{1}\left(\mathcal{U}\left(x_{1}\right) w_{1}, x_{1}\right) \cdots \mathcal{Y}_{i-1}\left(\mathcal{U}\left(x_{i-1}\right) w_{i-1}, x_{i-1}\right) \text {. } \\
& \text { - } \mathcal{Y}_{i}\left(\mathcal{U}\left(x_{1}\right) Y(u, y) w_{i}, x_{i}\right) \mathcal{Y}_{i+1}\left(\mathcal{U}\left(x_{i+1}\right) w_{i+1}, x_{i+1}\right) \text {. } \\
& \cdots \mathcal{Y}_{n}\left(\mathcal{U}\left(x_{n}\right) w_{n}, x_{n}\right) q^{L(0)} \\
& =\sum_{i=1}^{n} \sum_{m=0}^{\infty} \operatorname{tr}_{\tilde{W}_{n}}^{\phi} \frac{(2 \pi i)^{m+1}}{m !}\left(x_{i} \frac{\partial}{\partial x_{i}}\right)^{m} \delta\left(\frac{x}{x_{i}}\right) \text {. } \\
& \cdot \mathcal{Y}_{1}\left(\mathcal{U}\left(x_{1}\right) w_{1}, x_{1}\right) \cdots \mathcal{Y}_{i-1}\left(\mathcal{U}\left(x_{i-1}\right) w_{i-1}, x_{i-1}\right) \text {. } \\
& \text { - } \mathcal{Y}_{i}\left(\mathcal{U}\left(x_{1}\right) u_{m} w_{i}, x_{i}\right) \mathcal{Y}_{i+1}\left(\mathcal{U}\left(x_{i+1}\right) w_{i+1}, x_{i+1}\right) \text {. } \\
& \cdots \mathcal{Y}_{n}\left(\mathcal{U}\left(x_{n}\right) w_{n}, x_{n}\right) q^{L(0)} \\
& =\sum_{i=1}^{n} \sum_{m=0}^{\infty} \sum_{l=1}^{\infty} \frac{(2 \pi i)^{m+1}}{m !}\left(x_{i} \frac{\partial}{\partial x_{i}}\right)^{m}\left(\frac{x^{l}}{x_{i}^{l}}+\frac{x^{-l}}{x_{i}^{-l}}\right) \\
& \operatorname{tr}_{\tilde{W}_{n}}^{\phi} \mathcal{Y}_{1}\left(\mathcal{U}\left(x_{1}\right) w_{1}, x_{1}\right) \cdots \mathcal{Y}_{i-1}\left(\mathcal{U}\left(x_{i-1}\right) w_{i-1}, x_{i-1}\right) \text {. } \\
& \text { - } \mathcal{Y}_{i}\left(\mathcal{U}\left(x_{1}\right) u_{m} w_{i}, x_{i}\right) \mathcal{Y}_{i+1}\left(\mathcal{U}\left(x_{i+1}\right) w_{i+1}, x_{i+1}\right) \text {. } \\
& \cdots \mathcal{Y}_{n}\left(\mathcal{U}\left(x_{n}\right) w_{n}, x_{n}\right) q^{L(0)} \\
& +2 \pi i \sum_{i=1}^{n} \mathcal{Y}_{1}\left(\mathcal{U}\left(x_{1}\right) w_{1}, x_{1}\right) \cdots \mathcal{Y}_{i-1}\left(\mathcal{U}\left(x_{i-1}\right) w_{i-1}, x_{i-1}\right) \text {. } \\
& \cdot \mathcal{Y}_{i}\left(\mathcal{U}\left(x_{1}\right) u_{0} w_{i}, x_{i}\right) \mathcal{Y}_{i+1}\left(\mathcal{U}\left(x_{i+1}\right) w_{i+1}, x_{i+1}\right) \cdots \mathcal{Y}_{n}\left(\mathcal{U}\left(x_{n}\right) w_{n}, x_{n}\right) q^{L(0)}
\end{aligned}
$$

Since the operator $\left(1-q^{-x \frac{\partial}{\partial x}}\right)$ kills constant expressions in the variable $x$, the left hand side 
has no constant term as a series in $x$. This implies

$$
\begin{aligned}
\sum_{i=1}^{n} & \operatorname{tr}_{\tilde{W}_{n}}^{\phi} \mathcal{Y}_{1}\left(\mathcal{U}\left(x_{1}\right) w_{1}, x_{1}\right) \cdots \mathcal{Y}_{i-1}\left(\mathcal{U}\left(x_{i-1}\right) w_{i-1}, x_{i-1}\right) \\
& \cdot \mathcal{Y}_{i}\left(\mathcal{U}\left(x_{i}\right) u_{0} w_{i}, x_{i}\right) \mathcal{Y}_{i+1}\left(\mathcal{U}\left(x_{i+1}\right) w_{i+1}, x_{i+1}\right) \cdots \mathcal{Y}_{n}\left(\mathcal{U}\left(x_{n}\right) w_{n}, x_{n}\right) q^{L(0)} \\
& =0
\end{aligned}
$$

therefore,

$$
\begin{aligned}
& \left(1-q^{-x \frac{\partial}{\partial x}}\right) \operatorname{tr}_{\tilde{W}_{n}}^{\phi} Y(\mathcal{U}(x) u, x) \mathcal{Y}_{1}\left(\mathcal{U}\left(x_{1}\right) w_{1}, x_{1}\right) \cdots \mathcal{Y}_{n}\left(\mathcal{U}\left(x_{n}\right) w_{n}, x_{n}\right) q^{L(0)} \\
& =\sum_{i=1}^{n} \sum_{m=0}^{\infty} \sum_{l=1}^{\infty} \frac{(2 \pi i)^{m+1}}{m !}\left((-l)^{m} \frac{x^{l}}{x_{i}^{l}}+l^{m} \frac{x^{-l}}{x_{i}^{-l}}\right) \\
& \operatorname{tr}_{\tilde{W}_{n}}^{\phi} \mathcal{Y}_{1}\left(\mathcal{U}\left(x_{1}\right) w_{1}, x_{1}\right) \cdots \mathcal{Y}_{i-1}\left(\mathcal{U}\left(x_{i-1}\right) w_{i-1}, x_{i-1}\right) \text {. } \\
& \text { - } \mathcal{Y}_{i}\left(\mathcal{U}\left(x_{1}\right) u_{m} w_{i}, x_{i}\right) \mathcal{Y}_{i+1}\left(\mathcal{U}\left(x_{i+1}\right) w_{i+1}, x_{i+1}\right) \text {. } \\
& \cdots \mathcal{Y}_{n}\left(\mathcal{U}\left(x_{n}\right) w_{n}, x_{n}\right) q^{L(0)} \text {. }
\end{aligned}
$$

Then invert the operator $\left(1-q^{-x} \frac{\partial}{\partial x}\right)$, with the appropriate constant term:

$$
\begin{aligned}
\operatorname{tr}_{\tilde{W}_{n}}^{\phi} Y(\mathcal{U}(x) u, x) \mathcal{Y}_{1}\left(\mathcal{U}\left(x_{1}\right) w_{1}, x_{1}\right) \cdots \mathcal{Y}_{n}\left(\mathcal{U}\left(x_{n}\right) w_{n}, x_{n}\right) q^{L(0)} \\
=\operatorname{tr}_{\tilde{W}_{n}}^{\phi} o(U(1) u) \mathcal{Y}_{1}\left(\mathcal{U}\left(x_{1}\right) w_{1}, x_{1}\right) \cdots \mathcal{Y}_{n}\left(\mathcal{U}\left(x_{n}\right) w_{n}, x_{n}\right) q^{L(0)} \\
+\left(1-q^{-x} \frac{\partial}{\partial x}\right)^{-1} \sum_{i=1}^{n} \sum_{m=0}^{\infty} \sum_{l=1}^{\infty} \frac{(2 \pi i)^{m+1}}{m !}\left((-l)^{m} \frac{x^{l}}{x_{i}^{l}}+l^{m} \frac{x^{-l}}{x_{i}^{-l}}\right) \\
\operatorname{tr}_{\tilde{W}_{n}}^{\phi} \mathcal{Y}_{1}\left(\mathcal{U}\left(x_{1}\right) w_{1}, x_{1}\right) \cdots \mathcal{Y}_{i-1}\left(\mathcal{U}\left(x_{i-1}\right) w_{i-1}, x_{i-1}\right) . \\
\quad \cdot \mathcal{Y}_{i}\left(\mathcal{U}\left(x_{1}\right) u_{m} w_{i}, x_{i}\right) \mathcal{Y}_{i+1}\left(\mathcal{U}\left(x_{i+1}\right) w_{i+1}, x_{i+1}\right) \cdot \\
\quad \cdots \mathcal{Y}_{n}\left(\mathcal{U}\left(x_{n}\right) w_{n}, x_{n}\right) q^{L(0)} \\
=\operatorname{tr}_{\tilde{W}_{n}}^{\phi} o(U(1) u) \mathcal{Y}_{1}\left(\mathcal{U}\left(x_{1}\right) w_{1}, x_{1}\right) \cdots \mathcal{Y}_{n}\left(\mathcal{U}\left(x_{n}\right) w_{n}, x_{n}\right) q^{L(0)} \\
+\sum_{i=1}^{n} \sum_{m=0}^{\infty} \sum_{l=1}^{\infty} \frac{(2 \pi i)^{m+1}}{m !}\left(-(-l)^{m} \frac{q^{l} x^{l}}{\left(1-q^{l}\right) x_{i}^{l}}+l^{m} \frac{x^{-l}}{\left(1-q^{l}\right) x_{i}^{-l}}\right) \\
\operatorname{tr}_{\tilde{W}_{n}}^{\phi} \mathcal{Y}_{1}\left(\mathcal{U}\left(x_{1}\right) w_{1}, x_{1}\right) \cdots \mathcal{Y}_{i-1}\left(\mathcal{U}\left(x_{i-1}\right) w_{i-1}, x_{i-1}\right) \cdot \\
\quad \cdot \mathcal{Y}_{i}\left(\mathcal{U}\left(x_{1}\right) u_{m} w_{i}, x_{i}\right) \mathcal{Y}_{i+1}\left(\mathcal{U}\left(x_{i+1}\right) w_{i+1}, x_{i+1}\right) \cdot \\
\quad \cdots \mathcal{Y}_{n}\left(\mathcal{U}\left(x_{n}\right) w_{n}, x_{n}\right) q^{L(0)} \\
\operatorname{tr}_{\tilde{W}_{n}}^{\phi} o(U(1) u) \mathcal{Y}_{1}\left(\mathcal{U}\left(x_{1}\right) w_{1}, x_{1}\right) \cdots \mathcal{Y}_{n}\left(\mathcal{U}\left(x_{n}\right) w_{n}, x_{n}\right) q^{L(0)} \\
+\sum_{i=1}^{n} \sum_{m=0}^{\infty} \sum_{l=1}^{\infty} \frac{(2 \pi i)^{m+1}}{m !}\left(-(-l)^{m} \frac{q^{l}\left(\frac{x_{i}}{x}\right)^{-l}}{1-q^{l}}+l^{m} \frac{\left(\frac{x_{i}}{x}\right)^{l}}{\left(1-q^{l}\right)}\right)
\end{aligned}
$$




$$
\begin{aligned}
& \operatorname{tr}_{\tilde{W}_{n}}^{\phi} \mathcal{Y}_{1}\left(\mathcal{U}\left(x_{1}\right) w_{1}, x_{1}\right) \cdots \mathcal{Y}_{i-1}\left(\mathcal{U}\left(x_{i-1}\right) w_{i-1}, x_{i-1}\right) \\
& \cdot \mathcal{Y}_{i}\left(\mathcal{U}\left(x_{1}\right) u_{m} w_{i}, x_{i}\right) \mathcal{Y}_{i+1}\left(\mathcal{U}\left(x_{i+1}\right) w_{i+1}, x_{i+1}\right) \cdot \\
& \cdots \mathcal{Y}_{n}\left(\mathcal{U}\left(x_{n}\right) w_{n}, x_{n}\right) q^{L(0)} \\
&=\operatorname{tr}_{\tilde{W}_{n}}^{\phi} o(\mathcal{U}(1) u) \mathcal{Y}_{1}\left(\mathcal{U}\left(x_{1}\right) w_{1}, x_{1}\right) \cdots \mathcal{Y}_{n}\left(\mathcal{U}\left(x_{n}\right) w_{n}, x_{n}\right) q^{L(0)} \\
&+\sum_{i=1}^{n} \sum_{m \geq 0} P_{m+1}\left(\frac{x_{i}}{x} ; q\right) \operatorname{tr}_{\tilde{W}_{n}}^{\phi} \mathcal{Y}_{1}\left(\mathcal{U}\left(x_{1}\right) w_{1}, x_{1}\right) \cdot \\
& \quad \cdots \mathcal{Y}_{i-1}\left(\mathcal{U}\left(x_{i-1}\right) w_{i-1}, x_{i-1}\right) \mathcal{Y}_{i}\left(\mathcal{U}\left(x_{i}\right) u_{m} w_{i}, x_{i}\right) \cdot \\
& \cdot \mathcal{Y}_{i+1}\left(\mathcal{U}\left(x_{i+1}\right) w_{i+1}, x_{i+1}\right) \cdots \mathcal{Y}_{n}\left(\mathcal{U}\left(x_{n}\right) w_{n}, x_{n}\right) q^{L(0)}
\end{aligned}
$$

In the rest of this section, we consider grading-restricted generalized $V$-modules whose weights are real, and we make the following assumptions which will be proved in the next section:

- (Convergence of genus zero correlation functions) For any grading-restricted generalized $V$-modules $W_{i}, \tilde{W}_{i}, i=0, \ldots, n$ with $\tilde{W}_{n}=\tilde{W}_{0}$, for any elements $w_{i} \in W_{i}, \tilde{w}_{n} \in \tilde{W}_{n}$, $\tilde{w}_{n}^{\prime} \in \tilde{W}_{n}^{\prime}$, and for logarithmic intertwining operators $\mathcal{Y}_{i} \in\left(\begin{array}{c}\tilde{W}_{i-1} \\ W_{i} \tilde{W}_{i}\end{array}\right), i=0 \ldots n$, the series

$$
\left\langle\tilde{w}_{n}^{\prime}, \mathcal{Y}_{1}\left(w_{1}, z_{1}\right) \cdots \mathcal{Y}_{n}\left(w_{n}, z_{n}\right) \tilde{w}_{n}\right\rangle
$$

is absolutely convergent in the region $\left|z_{1}\right|>\left|z_{2}\right|>\ldots>\left|z_{n}\right|>0$.

- (Associativity for $P$-intertwining operators) For any grading-restricted generalized $V$ modules $\tilde{W}_{1}, \tilde{W}_{2}, V$-P-bimodules $W_{1}, W_{2}, W_{3}, P$-intertwining operators $\mathcal{Y}_{1} \in\left(\begin{array}{c}W_{2} \\ W_{2}, W_{3}\end{array}\right)$, $\mathcal{Y}_{2} \in\left(\begin{array}{c}W_{1} \\ \tilde{W}_{1}, W_{2}\end{array}\right)$ there exists a $V$-P-bimodule $M$, a logarithmic intertwining operator $Y_{3} \in\left(\begin{array}{c}M \\ \tilde{W}_{1}, \tilde{W}_{2}\end{array}\right)$ and a $P$-intertwining operator $\mathcal{Y}_{4} \in\left(\begin{array}{c}W_{1} \\ M, W_{3}\end{array}\right)$ such that for any complex numbers $z_{1}, z_{2}$ with $\left|z_{1}\right|>\left|z_{2}\right|>\left|z_{1}-z_{2}\right|>0$ and $\tilde{w}_{1} \in \tilde{W}_{1}, \tilde{w}_{2} \in W_{2}, w_{3} \in W_{3}$, $w_{1}^{\prime} \in W_{1}^{\prime}$

$$
\begin{aligned}
& \left\langle w_{1}^{\prime}, \mathcal{Y}_{1}\left(\tilde{w}_{1}, z_{1}\right) \mathcal{Y}_{2}\left(\tilde{w}_{2}, z_{2}\right) w_{3}\right\rangle \\
& \quad=\left\langle w_{1}^{\prime}, \mathcal{Y}_{4}\left(\mathcal{Y}_{3}\left(\tilde{w}_{1}, z_{1}-z_{2}\right) \tilde{w}_{2}, z_{2}\right) w_{3}\right\rangle
\end{aligned}
$$

- (Commutativity for $P$-intertwining operators) For any grading-restricted generalized $V$-modules $\tilde{W}_{1}, \tilde{W}_{2}, W_{1}, W_{2}, W_{3}, P$-intertwining operators $\mathcal{Y}_{1} \in\left(\begin{array}{c}W_{2} \\ \tilde{W}_{2}, W_{3}\end{array}\right), \mathcal{Y}_{2} \in\left(\begin{array}{c}W_{1} \\ \tilde{W}_{1}, W_{2}\end{array}\right)$ there exists a $V$-P-bimodule $M$ and $P$-intertwining operators $Y_{3} \in\left(\begin{array}{c}W_{1}, W \\ \tilde{W}_{2}, M\end{array}\right)$ and $\mathcal{Y}_{4} \in\left(\begin{array}{c}M \\ \tilde{W}_{1}, W_{3}\end{array}\right)$ such that for any $\tilde{w}_{1} \in \tilde{W}_{1}, \tilde{w}_{2} \in W_{2}, w_{3} \in W_{3}, w_{1}^{\prime} \in W_{1}^{\prime}$, the multivalued analytic function

$$
\left\langle w_{1}^{\prime}, \mathcal{Y}_{1}\left(\tilde{w}_{1}, z_{1}\right) \mathcal{Y}_{2}\left(\tilde{w}_{2}, z_{2}\right) w_{3}\right\rangle
$$


in the region $\left|z_{1}\right|>\left|z_{2}\right|>0$ is an analytic continuation of the multivalued analytic function

$$
\left\langle w_{1}^{\prime}, \mathcal{Y}_{3}\left(\tilde{w}_{2}, z_{2}\right) \mathcal{Y}_{4}\left(\tilde{w}_{1}, z_{1}\right) w_{3}\right\rangle
$$

in the region $\left|z_{2}\right|>\left|z_{1}\right|>0$.

Our goal is to obtain differential equations for the genus-one correlation functions. In order to do that, we need to derive formulae for

$$
\operatorname{tr}_{\tilde{W}_{n}}^{\phi} \mathcal{Y}_{1}\left(\mathcal{U}\left(x_{1}\right) w_{1}, x_{1}\right) \cdots \mathcal{Y}_{i}\left(\mathcal{U}\left(x_{i}\right) L(-1) w_{i}, x_{i}\right) \cdots \mathcal{Y}_{n}\left(\mathcal{U}\left(x_{n}\right) w_{n}, x_{n}\right) q^{L(0)}
$$

which is related to the derivative of the formal $q$-trace with respect to the variable $x_{i}$; hence, we consider the expression

$$
\operatorname{tr}_{\tilde{W}_{n}}^{\phi} \mathcal{Y}_{1}\left(\mathcal{U}\left(x_{1}\right) w_{1}, x_{1}\right) \cdots \mathcal{Y}_{i}\left(\mathcal{U}\left(x_{i}\right) Y(u, y) w_{i}, x_{i}\right) \cdots \mathcal{Y}_{n}\left(\mathcal{U}\left(x_{n}\right) w_{n}, x_{n}\right) q^{L(0)}
$$

Using the $\mathcal{U}(x)$ conjugation property, one can rewrite this as

$$
\begin{aligned}
\operatorname{tr}_{\tilde{W}_{n}}^{\phi} \mathcal{Y}_{1}\left(\mathcal{U}\left(x_{1}\right) w_{1}, x_{1}\right) \cdot \\
\cdots \mathcal{Y}_{i}\left(Y\left(\mathcal{U}\left(x_{i} e^{2 \pi i y} u, x_{i}\left(e^{2 \pi i y}-1\right)\right) \mathcal{U}\left(x_{i}\right) w_{i}, x_{i}\right)\right. \\
\quad \cdots \mathcal{Y}_{n}\left(\mathcal{U}\left(x_{n}\right) w_{n}, x_{n}\right) q^{L(0)}
\end{aligned}
$$

then, in order to use (1.9), one rewrites the iterate as a product using associativity for intertwining operators.

Lemma 1.27. Let $\mathcal{Y}$ be a logarithmic intertwining operator of type $\left(\begin{array}{c}W_{0} \\ W W_{1}\end{array}\right)$ for gradingrestricted generalized $V$-modules $W, W_{0}, W_{1}$. Then for any $w_{0}^{\prime} \in W_{0}^{\prime}, w_{1} \in W_{1}, w \in W$, and for any complex number $z$ satisfying $\left|q_{z}\right|>1>\left|q_{z}-1\right|>0$,

$$
\begin{array}{r}
\left\langle w_{0}^{\prime}, \mathcal{Y}\left(Y\left(\mathcal{U}\left(x q_{z}\right) u, x\left(q_{z}-1\right)\right) \mathcal{U}(x) w, x\right) w_{1}\right\rangle \\
=\left\langle w_{0}^{\prime}, Y\left(\mathcal{U}\left(x q_{z}\right) u, x q_{z}\right) \mathcal{Y}(\mathcal{U}(x) w, x) w_{1}\right\rangle
\end{array}
$$

Proof. Using associativity for intertwining operators, we see that

$$
\begin{array}{r}
\left\langle w_{0}^{\prime}, \mathcal{Y}\left(Y\left(\mathcal{U}\left(z_{1} q_{z}\right) u, z_{1}\left(q_{z}-1\right)\right) \mathcal{U}\left(z_{1}\right) w, z_{1}\right) w_{1}\right\rangle \\
=\left\langle w_{0}^{\prime}, Y\left(\mathcal{U}\left(z_{1} q_{z}\right) u, z_{1} q_{z}\right) \mathcal{Y}\left(\mathcal{U}\left(z_{1}\right) w, z_{1}\right) w_{1}\right\rangle
\end{array}
$$

holds for any complex numbers $z, z_{1}$ in the region $\left|z_{1} q_{z}\right|>\left|z_{1}\right|>\left|z_{1}\left(q_{z}-1\right)\right|>0$, or whenever $z_{1} \neq 0$ and $\left|q_{z}\right|>1>\left|q_{z}-1\right|>0$. Then for a fixed $z$ in the above region, we have two series in powers of $z_{1}$ (not necessarily integral), and $\log z_{1}$; by our assumption on the modules, these powers must form a unique expansion set, and thus the coefficients of the two series must be equal. Then, we can replace the complex variable $z_{1}$ in (1.12) with the formal variable $x$, which concludes the proof. 
Proposition 1.28. Consider grading-restricted generalized $V$-modules $W_{i}$ and $V$-P-bimodules $\tilde{W}_{i}$ for $i=1, \ldots, n$, with $\tilde{W}_{0}=\tilde{W}_{n}$, and P-intertwining operators $\mathcal{Y}_{i}$ of type $\left(\begin{array}{l}\tilde{W}_{i-1} \\ W_{i} \tilde{W}_{i}\end{array}\right)$ for $i=1, \ldots, n$. Moreover, suppose $\tilde{W}_{0}$ is projective as right $P$-module. Then for any $v \in V$, $w_{i} \in W_{i}$, and any integer $j, 1 \leq j \leq n$,

$$
\begin{aligned}
& \operatorname{tr}_{\tilde{W}_{n}}^{\phi} \mathcal{Y}_{1}\left(\mathcal{U}\left(x_{1}\right) w_{1}, x_{1}\right) \cdots \mathcal{Y}_{j-1}\left(\mathcal{U}\left(x_{j-1}\right) w_{j-1}, x_{j-1}\right) \mathcal{Y}_{j}\left(\mathcal{U}\left(x_{j}\right) Y(v, y) w_{j}, x_{j}\right) \\
& \cdot \mathcal{Y}_{j+1}\left(\mathcal{U}\left(x_{j+1}\right) w_{j+1}, x_{j+1}\right) \cdots \mathcal{Y}_{n}\left(\mathcal{U}\left(x_{n}\right) w_{n}, x_{n}\right) q^{L(0)} \\
& =\sum_{m \geq 0}(-1)^{m+1}\left(\tilde{\wp}_{m+1}(-y ; q)+\frac{\partial^{m}}{\partial y^{m}}\left(\tilde{G}_{2}(q) y+\pi i\right)\right) \text {. } \\
& \operatorname{tr}_{\tilde{W}_{n}}^{\phi} \mathcal{Y}_{1}\left(\mathcal{U}\left(x_{1}\right) w_{1}, x_{1}\right) \cdots \mathcal{Y}_{j-1}\left(\mathcal{U}\left(x_{j-1}\right) w_{j-1}, x_{j-1}\right) . \\
& \text { - } \mathcal{Y}_{j}\left(\mathcal{U}\left(x_{j}\right) v_{m} w_{j}, x_{j}\right) \mathcal{Y}_{j+1}\left(\mathcal{U}\left(x_{j+1}\right) w_{j+1}, x_{j+1}\right) \text {. } \\
& \cdots \mathcal{Y}_{n}\left(\mathcal{U}\left(x_{n}\right) w_{n}, x_{n}\right) q^{L(0)} \\
& +\sum_{i \neq j} \sum_{m \geq 0} P_{m+1}\left(\frac{x_{i}}{x_{j} e^{2 \pi i y}} ; q\right) \text {. } \\
& \cdot \operatorname{tr}_{\tilde{W}_{n}}^{\phi} \mathcal{Y}_{1}\left(\mathcal{U}\left(x_{1}\right) w_{1}, x_{1}\right) \cdots \mathcal{Y}_{i-1}\left(\mathcal{U}\left(x_{i-1}\right) w_{i-1}, x_{i-1}\right) \text {. } \\
& \text { - } \mathcal{Y}_{i}\left(\mathcal{U}\left(x_{i}\right) v_{m} w_{i}, x_{i}\right) \mathcal{Y}_{i+1}\left(\mathcal{U}\left(x_{i+1}\right) w_{i+1}, x_{i+1}\right) \cdots \mathcal{Y}_{n}\left(\mathcal{U}\left(x_{n}\right) w_{n}, x_{n}\right) q^{L(0)} \\
& +\operatorname{tr}_{\tilde{W}_{n}}^{\phi} o(\mathcal{U}(1) v) \mathcal{Y}_{1}\left(\mathcal{U}\left(x_{1}\right) w_{1}, x_{1}\right) \cdots \mathcal{Y}_{n}\left(\mathcal{U}\left(x_{n}\right) w_{n}, x_{n}\right) q^{L(0)} .
\end{aligned}
$$

Proof. By induction on $j$; when $j=1$, by Lemma 1.8,

$$
\begin{aligned}
\operatorname{tr}_{\tilde{W}_{n}}^{\phi} \mathcal{Y}_{1}\left(\mathcal{U}\left(x_{1}\right) Y(v, y) w_{1}, x_{1}\right) \mathcal{Y}_{2}\left(\mathcal{U}\left(x_{2}\right) w_{2}, x_{2}\right) \cdots \mathcal{Y}_{n}\left(\mathcal{U}\left(x_{n}\right) w_{n}, x_{n}\right) q^{L(0)} & \\
=\operatorname{tr}_{\tilde{W}_{n}}^{\phi} & \mathcal{Y}_{1}\left(Y\left(\mathcal{U}\left(x_{1} e^{2 \pi i y}\right) v, x_{1}\left(e^{2 \pi i y}-1\right)\right) \mathcal{U}\left(x_{1}\right) w_{1}, x_{1}\right) \\
& \cdot \mathcal{Y}_{2}\left(\mathcal{U}\left(x_{2}\right) w_{2}, x_{2}\right) \cdots \mathcal{Y}_{n}\left(\mathcal{U}\left(x_{n}\right) w_{n}, x_{n}\right) q^{L(0)}
\end{aligned}
$$

and using (1.11), for any complex number $z$ such that $\left|q_{z}\right|>1>\left|q_{z}-1\right|>0$,

$$
\begin{aligned}
& \operatorname{tr}_{\tilde{W}_{n}}^{\phi} \mathcal{Y}_{1}\left(\mathcal{U}\left(x_{1}\right) Y(v, z) w_{1}, x_{1}\right) \mathcal{Y}_{2}\left(\mathcal{U}\left(x_{2}\right) w_{2}, x_{2}\right) \cdots \mathcal{Y}_{n}\left(\mathcal{U}\left(x_{n}\right) w_{n}, x_{n}\right) q^{L(0)} \\
&=\operatorname{tr}_{\tilde{W}_{n}}^{\phi} \mathcal{Y}_{1}\left(Y\left(\mathcal{U}\left(x_{1} q_{z}\right) v, x_{1}\left(q_{z}-1\right)\right) \mathcal{U}\left(x_{1}\right) w_{1}, x_{1}\right) \\
& \cdot \mathcal{Y}_{2}\left(\mathcal{U}\left(x_{2}\right) w_{2}, x_{2}\right) \cdots \mathcal{Y}_{n}\left(\mathcal{U}\left(x_{n}\right) w_{n}, x_{n}\right) q^{L(0)} \\
&=\operatorname{tr}_{\tilde{W}_{n}}^{\phi} Y\left(\mathcal{U}\left(x_{1} q_{z}\right) v, x_{1} q_{z}\right) \mathcal{Y}_{1}\left(\mathcal{U}\left(x_{1}\right) w_{1}, x_{1}\right) \\
& \cdot \mathcal{Y}_{2}\left(\mathcal{U}\left(x_{2}\right) w_{2}, x_{2}\right) \cdots \mathcal{Y}_{n}\left(\mathcal{U}\left(x_{n}\right) w_{n}, x_{n}\right) q^{L(0)}
\end{aligned}
$$

Now by (1.9) with $x=x_{1} q_{z}$, we get

$$
\begin{aligned}
& \operatorname{tr}_{\tilde{W}_{n}}^{\phi} \mathcal{Y}_{1}\left(\mathcal{U}\left(x_{1}\right) Y(v, z) w_{1}, x_{1}\right) \mathcal{Y}_{2}\left(\mathcal{U}\left(x_{2}\right) w_{2}, x_{2}\right) \cdots \mathcal{Y}_{n}\left(\mathcal{U}\left(x_{n}\right) w_{n}, x_{n}\right) q^{L(0)} \\
& \quad=\sum_{m \geq 0} P_{m+1}\left(\frac{1}{q_{z}} ; q\right) \operatorname{tr}_{\tilde{W}_{n}}^{\phi} \mathcal{Y}_{1}\left(\mathcal{U}\left(x_{1}\right) v_{m} w_{1}, x_{1}\right) .
\end{aligned}
$$




$$
\begin{aligned}
& \cdot \mathcal{Y}_{2}\left(\mathcal{U}\left(x_{2}\right) w_{2}, x_{2}\right) \cdots \mathcal{Y}_{n}\left(\mathcal{U}\left(x_{n}\right) w_{n}, x_{n}\right) q^{L(0)} \\
&+ \sum_{i=2}^{m} \sum_{m \geq 0} P_{m+1}\left(\frac{x_{i}}{x_{1} q_{z}} ; q\right) \operatorname{tr}_{\tilde{W}_{n}}^{\phi} \mathcal{Y}_{1}\left(\mathcal{U}\left(x_{1}\right) w_{1}, x_{1}\right) \cdots \mathcal{Y}_{i-1}\left(\mathcal{U}\left(x_{i-1}\right) w_{i-1}, x_{i-1}\right) \\
& \cdot \mathcal{Y}_{i}\left(\mathcal{U}\left(x_{1}\right) v_{m} w_{i}, x_{i}\right) \cdot \mathcal{Y}_{i+1}\left(\mathcal{U}\left(x_{i+1}\right) w_{i+1}, x_{i+1}\right) \cdots \mathcal{Y}_{n}\left(\mathcal{U}\left(x_{n}\right) w_{n}, x_{n}\right) q^{L(0)} \\
&+ \operatorname{tr}_{\tilde{W}_{n}}^{\phi} o(\mathcal{U}(1) v) \mathcal{Y}_{1}\left(\mathcal{U}\left(x_{1}\right) w_{1}, x_{1}\right) \cdots \mathcal{Y}_{n}\left(\mathcal{U}\left(x_{n}\right) w_{n}, x_{n}\right) q^{L(0)} \\
&=\sum_{m \geq 0}(-1)^{m+1}\left(\tilde{\wp}_{m+1}(-z ; q)+\frac{\partial^{m}}{\partial z^{m}}\left(\tilde{G}_{2}(q) z+\pi i\right)\right) \operatorname{tr}_{\tilde{W}_{n}}^{\phi} \mathcal{Y}_{1}\left(\mathcal{U}\left(x_{1}\right) v_{m} w_{1}, x_{1}\right) \\
& \quad \cdot \mathcal{Y}_{2}\left(\mathcal{U}\left(x_{2}\right) w_{2}, x_{2}\right) \cdots \mathcal{Y}_{n}\left(\mathcal{U}\left(x_{n}\right) w_{n}, x_{n}\right) q^{L(0)} \\
&+\sum_{i=2}^{m} \sum_{m \geq 0} P_{m+1}\left(\frac{x_{i}}{x_{1} q_{z}} ; q\right) \operatorname{tr}_{\tilde{W}_{n}}^{\phi} \mathcal{Y}_{1}\left(\mathcal{U}\left(x_{1}\right) w_{1}, x_{1}\right) \cdots \mathcal{Y}_{i-1}\left(\mathcal{U}\left(x_{i-1}\right) w_{i-1}, x_{i-1}\right) \\
& \quad \cdot \mathcal{Y}_{i}\left(\mathcal{U}\left(x_{1}\right) v_{m} w_{i}, x_{i}\right) \cdot \mathcal{Y}_{i+1}\left(\mathcal{U}\left(x_{i+1}\right) w_{i+1}, x_{i+1}\right) \cdots \mathcal{Y}_{n}\left(\mathcal{U}\left(x_{n}\right) w_{n}, x_{n}\right) q^{L(0)} \\
&+\operatorname{tr}_{\tilde{W}_{n}}^{\phi} o(\mathcal{U}(1) v) \mathcal{Y}_{1}\left(\mathcal{U}\left(x_{1}\right) w_{1}, x_{1}\right) \cdots \mathcal{Y}_{n}\left(\mathcal{U}\left(x_{n}\right) w_{n}, x_{n}\right) q^{L(0)}
\end{aligned}
$$

which proves the base case. Now suppose the result holds for $j \geq 1$, and use commutativity for intertwining operators.

Proposition 1.29. Using the same notation as in the previous proposition, for all $v \in V$ and $l \geq 1$, we have

$$
\begin{aligned}
\operatorname{tr}_{\tilde{W}_{n}}^{\phi} \mathcal{Y}_{1}\left(\mathcal{U}\left(q_{z_{1}}\right) w_{1}, q_{z_{1}}\right) \cdots \mathcal{Y}_{j-1}\left(\mathcal{U}\left(q_{z_{j-1}}\right) w_{j-1}, q_{z_{j-1}}\right) \mathcal{Y}_{j}\left(\mathcal{U}\left(q_{z_{j}}\right) v_{-l} w_{j}, q_{z_{j}}\right) & \cdot \mathcal{Y}_{i+1}\left(\mathcal{U}\left(q_{z_{i+1}}\right) w_{i+1}, q_{z_{i+1}}\right) \cdots \mathcal{Y}_{n}\left(\mathcal{U}\left(q_{z_{n}}\right) w_{n}, q_{z_{n}}\right) q^{L(0)} \\
= & \sum_{k=1}^{\infty}(-1)^{l+1}\left(\begin{array}{c}
2 k+1 \\
l-1
\end{array}\right) \tilde{G}_{2 k+2}(q) \operatorname{tr}_{\tilde{W}_{n}}^{\phi} \mathcal{Y}_{1}\left(\mathcal{U}\left(q_{z_{1}}\right) w_{1}, q_{z_{1}}\right) \\
& \ldots \mathcal{Y}_{j-1}\left(\mathcal{U}\left(q_{z_{j-1}}\right) w_{j-1}, q_{z_{j-1}}\right) \mathcal{Y}_{j}\left(\mathcal{U}\left(q_{z_{j}}\right) v_{2 k+2-l} w_{j}, q_{z_{j}}\right) \cdot \\
& \cdot \mathcal{Y}_{i+1}\left(\mathcal{U}\left(q_{z_{i+1}}\right) w_{i+1}, q_{z_{i+1}}\right) \cdots \mathcal{Y}_{n}\left(\mathcal{U}\left(q_{z_{n}}\right) w_{n}, q_{z_{n}}\right) q^{L(0)} \\
+ & \sum_{i \neq j} \sum_{m=0}^{\infty}(-1)^{m+l}\left(\begin{array}{c}
-m-1 \\
l-1
\end{array}\right) \tilde{\wp}_{m+l}\left(z_{i}-z_{j} ; q\right) \operatorname{tr}_{\tilde{W}_{n}}^{\phi} \mathcal{Y}_{1}\left(\mathcal{U}\left(q_{z_{1}}\right) w_{1}, q_{z_{1}}\right) \\
& \quad \cdot \mathcal{Y}_{i-1}\left(\mathcal{U}\left(q_{z_{i-1}}\right) w_{i-1}, q_{z_{i-1}}\right) \mathcal{Y}_{i}\left(\mathcal{U}\left(q_{z_{i}}\right) v_{m} w_{i}, q_{z_{i}}\right) \\
& \cdot \mathcal{Y}_{i+1}\left(\mathcal{U}\left(q_{z_{i+1}}\right) w_{i+1}, q_{z_{i+1}}\right) \cdots \mathcal{Y}_{n}\left(\mathcal{U}\left(q_{z_{n}}\right) w_{n}, q_{z_{n}}\right) q^{L(0)} \\
+ & \delta_{l, 1} \tilde{G}_{2}(q) \sum_{i=1}^{n} \operatorname{tr}_{\tilde{W}_{n}}^{\phi} \mathcal{Y}_{1}\left(\mathcal{U}\left(q_{z_{1}}\right) w_{1}, q_{z_{1}}\right) \cdots \mathcal{Y}_{i-1}\left(\mathcal{U}\left(q_{z_{i-1}}\right) w_{i-1}, q_{z_{i-1}}\right) \\
& \cdot \mathcal{Y}_{i}\left(\mathcal{U}\left(q_{z_{i}}\right)\left(v_{1}+v_{0} z_{i}\right) w_{i}, q_{z_{i}}\right) \mathcal{Y}_{i+1}\left(\mathcal{U}\left(q_{z_{i+1}}\right) w_{i+1}, q_{z_{i+1}}\right) \\
& \ldots \mathcal{Y}_{n}\left(\mathcal{U}\left(q_{z_{n}}\right) w_{n}, q_{z_{n}}\right) q^{L(0)} \\
+ & \delta_{l, 1} \operatorname{tr}_{\tilde{W}_{n}}^{\phi} o(\mathcal{U}(1) v) \mathcal{Y}_{1}\left(\mathcal{U}\left(q_{z_{1}}\right) w_{1}, q_{z_{1}}\right) \cdots \mathcal{Y}_{n}\left(\mathcal{U}\left(q_{z_{n}}\right) w_{n}, q_{z_{n}}\right) q^{L(0)}
\end{aligned}
$$


Proof. Notice that the coefficients of (1.13) as a series in the formal variables $q, \log q$ are absolutely convergent in the region $\left|q_{z_{1}}\right|>\ldots>\left|q_{z_{n}}\right|>0$. Since the $q$ coefficients of $\tilde{\wp}_{m}(z, q)$ are absolutely convergent when $|z|<1$, we can substitute $y=z, x_{i}=q_{z_{i}}$, for $i=1, \ldots, n$ in (1.13),

$$
\begin{aligned}
\operatorname{tr}_{\tilde{W}_{n}}^{\phi} \mathcal{Y}_{1}\left(\mathcal{U}\left(q_{z_{1}}\right) w_{1}, q_{z_{1}}\right) \cdots \mathcal{Y}_{j-1}\left(\mathcal{U}\left(q_{z_{j-1}}\right) w_{j-1}, q_{z_{j-1}}\right) \mathcal{Y}_{j}\left(\mathcal{U}\left(q_{z_{j}}\right) Y(v, z) w_{j}, q_{z_{j}}\right) \\
\cdot \mathcal{Y}_{j+1}\left(\mathcal{U}\left(q_{z_{j+1}}\right) w_{j+1}, q_{z_{j+1}}\right) \cdots \mathcal{Y}_{n}\left(\mathcal{U}\left(q_{z_{n}}\right) w_{n}, q_{z_{n}}\right) q^{L(0)} \\
=\sum_{m \geq 0}(-1)^{m+1}\left(\tilde{\wp}_{m+1}(-z ; q)+\frac{\partial^{m}}{\partial z^{m}}\left(\tilde{G}_{2}(q) z+\pi i\right)\right) \cdot \\
\operatorname{tr}_{\tilde{W}_{n}}^{\phi} \mathcal{Y}_{1}\left(\mathcal{U}\left(q_{z_{1}}\right) w_{1}, q_{z_{1}}\right) \cdots \mathcal{Y}_{j-1}\left(\mathcal{U}\left(q_{z_{j-1}}\right) w_{j-1}, q_{z_{j-1}}\right) \cdot \\
\cdot \mathcal{Y}_{j}\left(\mathcal{U}\left(q_{z_{j}}\right) v_{m} w_{j}, q_{z_{j}}\right) \mathcal{Y}_{j+1}\left(\mathcal{U}\left(q_{z_{j+1}}\right) w_{j+1}, q_{z_{j+1}}\right) \cdot \\
\quad \cdots \mathcal{Y}_{n}\left(\mathcal{U}\left(q_{z_{n}}\right) w_{n}, q_{z_{n}}\right) q^{L(0)} \\
\left.+\sum_{i \neq j} \sum_{m \geq 0} \tilde{(}-1\right)^{m+1}\left(\wp_{m+1}\left(z_{i}-z_{j}-z ; q\right)\right. \\
\left.\quad+(-1)^{m+1} \frac{\partial^{m}}{\partial z_{i}^{m}}\left(\tilde{G}_{2}(q)\left(z_{i}-z_{j}-z\right)-\pi i\right)\right) . \\
\cdot \operatorname{tr}_{\tilde{W}_{n}}^{\phi} \mathcal{Y}_{1}\left(\mathcal{U}\left(q_{z_{1}}\right) w_{1}, q_{z_{1}}\right) \cdots \mathcal{Y}_{i-1}\left(\mathcal{U}\left(q_{z_{i-1}}\right) w_{i-1}, q_{z_{i-1}}\right) \cdot \\
\cdot \mathcal{Y}_{i}\left(\mathcal{U}\left(q_{z_{i}}\right) v_{m} w_{i}, q_{z_{i}}\right) \mathcal{Y}_{i+1}\left(\mathcal{U}\left(q_{z_{i+1}}\right) w_{i+1}, q_{z_{i+1}}\right) \cdots \mathcal{Y}_{n}\left(\mathcal{U}\left(q_{z_{n}}\right) w_{n}, q_{z_{n}}\right) q^{L(0)} \\
+\operatorname{tr}_{\tilde{W}_{n}}^{\phi} o(\mathcal{U}(1) v) \mathcal{Y}_{1}\left(\mathcal{U}\left(q_{z_{1}}\right) w_{1}, q_{z_{1}}\right) \cdots \mathcal{Y}_{n}\left(\mathcal{U}\left(q_{z_{n}}\right) w_{n}, q_{z_{n}}\right) q^{L(0)} .
\end{aligned}
$$

The result follows by taking the coefficient of $z^{l-1}$ and using the $q$ expansion of $\tilde{\wp}(z ; q)$ and (1.10).

Taking $v=\omega$ and $l=1$ in (1.14), one obtains

$$
\begin{aligned}
\operatorname{tr}_{\tilde{W}_{n}}^{\phi} \mathcal{Y}_{1}\left(\mathcal{U}\left(q_{z_{1}}\right) w_{1}, q_{z_{1}}\right) \cdots \mathcal{Y}_{j-1}\left(\mathcal{U}\left(q_{z_{j-1}}\right) w_{j-1}, q_{z_{j-1}}\right) \mathcal{Y}_{j}\left(\mathcal{U}\left(q_{z_{j}}\right) \omega_{-1} w_{j}, q_{z_{j}}\right) \\
\quad \cdot \mathcal{Y}_{i+1}\left(\mathcal{U}\left(q_{z_{i+1}}\right) w_{i+1}, q_{z_{i+1}}\right) \cdots \mathcal{Y}_{n}\left(\mathcal{U}\left(q_{z_{n}}\right) w_{n}, q_{z_{n}}\right) q^{L(0)} \\
=\sum_{k=1}^{\infty} \tilde{G}_{2 k+2}(q) \operatorname{tr}_{\tilde{W}_{n}}^{\phi} \mathcal{Y}_{1}\left(\mathcal{U}\left(q_{z_{1}}\right) w_{1}, q_{z_{1}}\right) \\
\quad \cdots \mathcal{Y}_{j-1}\left(\mathcal{U}\left(q_{z_{j-1}}\right) w_{j-1}, q_{z_{j-1}}\right) \mathcal{Y}_{j}\left(\mathcal{U}\left(q_{z_{j}}\right) \omega_{2 k+1} w_{j}, q_{z_{j}}\right) \\
\quad \cdot \mathcal{Y}_{i+1}\left(\mathcal{U}\left(q_{z_{i+1}}\right) w_{i+1}, q_{z_{i+1}}\right) \cdots \mathcal{Y}_{n}\left(\mathcal{U}\left(q_{z_{n}}\right) w_{n}, q_{z_{n}}\right) q^{L(0)} \\
+\sum_{i \neq j} \sum_{m=0}^{\infty}(-1)^{m+1} \tilde{\wp}_{m+1}\left(z_{i}-z_{j} ; q\right) \operatorname{tr}_{\tilde{W}_{n}}^{\phi} \mathcal{Y}_{1}\left(\mathcal{U}\left(q_{z_{1}}\right) w_{1}, q_{z_{1}}\right) \\
\quad \cdots \mathcal{Y}_{i-1}\left(\mathcal{U}\left(q_{z_{i-1}}\right) w_{i-1}, q_{z_{i-1}}\right) \mathcal{Y}_{i}\left(\mathcal{U}\left(q_{z_{i}}\right) \omega_{m} w_{i}, q_{z_{i}}\right) \\
\quad \cdot \mathcal{Y}_{i+1}\left(\mathcal{U}\left(q_{z_{i+1}}\right) w_{i+1}, q_{z_{i+1}}\right) \cdots \mathcal{Y}_{n}\left(\mathcal{U}\left(q_{z_{n}}\right) w_{n}, q_{z_{n}}\right) q^{L(0)}
\end{aligned}
$$




$$
\begin{aligned}
+ & \tilde{G}_{2}(q) \sum_{i=1}^{n} \operatorname{tr}_{\tilde{W}_{n}}^{\phi} \mathcal{Y}_{1}\left(\mathcal{U}\left(q_{z_{1}}\right) w_{1}, q_{z_{1}}\right) \cdots \mathcal{Y}_{i-1}\left(\mathcal{U}\left(q_{z_{i-1}}\right) w_{i-1}, q_{z_{i-1}}\right) \\
& \cdot \mathcal{Y}_{i}\left(\mathcal{U}\left(q_{z_{i}}\right)\left(\omega_{1}+\omega_{0} z_{i}\right) w_{i}, q_{z_{i}}\right) \mathcal{Y}_{i+1}\left(\mathcal{U}\left(q_{z_{i+1}}\right) w_{i+1}, q_{z_{i+1}}\right) \\
& \cdots \mathcal{Y}_{n}\left(\mathcal{U}\left(q_{z_{n}}\right) w_{n}, q_{z_{n}}\right) q^{L(0)} \\
+ & \operatorname{tr}_{\tilde{W}_{n}}^{\phi} o(\mathcal{U}(1) \omega) \mathcal{Y}_{1}\left(\mathcal{U}\left(q_{z_{1}}\right) w_{1}, q_{z_{1}}\right) \cdots \mathcal{Y}_{n}\left(\mathcal{U}\left(q_{z_{n}}\right) w_{n}, q_{z_{n}}\right) q^{L(0)}
\end{aligned}
$$

now since $w_{k}=L(k-1)$, and since $\mathcal{U}(1) \omega=(2 \pi i)^{2}\left(\omega-\frac{c}{24} \mathbf{1}\right)$, we obtain

$$
\begin{aligned}
\operatorname{tr}_{\tilde{W}_{n}}^{\phi} \mathcal{Y}_{1}( & \left.\mathcal{U}\left(q_{z_{1}}\right) w_{1}, q_{z_{1}}\right) \cdots \mathcal{Y}_{j-1}\left(\mathcal{U}\left(q_{z_{j-1}}\right) w_{j-1}, q_{z_{j-1}}\right) \mathcal{Y}_{j}\left(\mathcal{U}\left(q_{z_{j}}\right) L(-2) w_{j}, q_{z_{j}}\right) \\
& \cdot \mathcal{Y}_{i+1}\left(\mathcal{U}\left(q_{z_{i+1}}\right) w_{i+1}, q_{z_{i+1}}\right) \cdots \mathcal{Y}_{n}\left(\mathcal{U}\left(q_{z_{n}}\right) w_{n}, q_{z_{n}}\right) q^{L(0)} \\
= & \sum_{k=1}^{\infty} \tilde{G}_{2 k+2}(q) \operatorname{tr}_{\tilde{W}_{n}}^{\phi} \mathcal{Y}_{1}\left(\mathcal{U}\left(q_{z_{1}}\right) w_{1}, q_{z_{1}}\right) \\
& \cdots \mathcal{Y}_{j-1}\left(\mathcal{U}\left(q_{z_{j-1}}\right) w_{j-1}, q_{z_{j-1}}\right) \mathcal{Y}_{j}\left(\mathcal{U}\left(q_{z_{j}}\right) L(2 k) w_{j}, q_{z_{j}}\right) \\
& \cdot \mathcal{Y}_{i+1}\left(\mathcal{U}\left(q_{z_{i+1}}\right) w_{i+1}, q_{z_{i+1}}\right) \cdots \mathcal{Y}_{n}\left(\mathcal{U}\left(q_{z_{n}}\right) w_{n}, q_{z_{n}}\right) q^{L(0)} \\
+ & \sum_{i \neq j} \sum_{m=0}^{\infty}(-1)^{m+1} \tilde{\wp}_{m+1}\left(z_{i}-z_{j} ; q\right) \operatorname{tr}_{\tilde{W}_{n}}^{\phi} \mathcal{Y}_{1}\left(\mathcal{U}\left(q_{z_{1}}\right) w_{1}, q_{z_{1}}\right) \\
& \cdots \mathcal{Y}_{i-1}\left(\mathcal{U}\left(q_{z_{i-1}}\right) w_{i-1}, q_{z_{i-1}}\right) \mathcal{Y}_{i}\left(\mathcal{U}\left(q_{z_{i}}\right) L(m-1) w_{i}, q_{z_{i}}\right) \\
& \cdot \mathcal{Y}_{i+1}\left(\mathcal{U}\left(q_{z_{i+1}}\right) w_{i+1}, q_{z_{i+1}}\right) \cdots \mathcal{Y}_{n}\left(\mathcal{U}\left(q_{z_{n}}\right) w_{n}, q_{z_{n}}\right) q^{L(0)} \\
+ & \tilde{G}_{2}(q) \sum_{i=1}^{n} \operatorname{tr}_{\tilde{W}_{n}}^{\phi} \mathcal{Y}_{1}\left(\mathcal{U}\left(q_{z_{1}}\right) w_{1}, q_{z_{1}}\right) \cdots \mathcal{Y}_{i-1}\left(\mathcal{U}\left(q_{z_{i-1}}\right) w_{i-1}, q_{z_{i-1}}\right) \\
& \cdot \mathcal{Y}_{i}\left(\mathcal{U}\left(q_{z_{i}}\right)\left(L(0)+L(-1) z_{i}\right) w_{i}, q_{z_{i}}\right) \mathcal{Y}_{i+1}\left(\mathcal{U}\left(q_{z_{i+1}}\right) w_{i+1}, q_{z_{i+1}}\right) \\
& \cdots \mathcal{Y}_{n}\left(\mathcal{U}\left(q_{z_{n}}\right) w_{n}, q_{z_{n}}\right) q^{L(0)} \\
+ & (2 \pi i)^{2} \operatorname{tr}_{\tilde{W}_{n}}^{\phi}\left(L(0)-\frac{c}{24}\right) \cdot \\
& \cdot \mathcal{Y}_{1}\left(\mathcal{U}\left(q_{z_{1}}\right) w_{1}, q_{z_{1}}\right) \cdots \mathcal{Y}_{n}\left(\mathcal{U}\left(q_{z_{n}}\right) w_{n}, q_{z_{n}}\right) q^{L(0)}
\end{aligned}
$$

\subsection{Duality properties for $P$-intertwining operators}

In this section we derive associativity and commutativity properties for $P$-intertwining operators, under the same assumptions as Proposition 1.10, we show that in the statement of these two properties, all the modules can be taken to be $V$ - $P$-bimodules and all intertwining operators to be $P$-intertwining operators. In particular, under those assumptions all identities in the previous sections hold.

Let $W_{1}$ be a grading-restricted generalized $V$-module and $W_{2}$ be a $V$ - $P$-bimodule. We can define a right action of $P$ on $W_{1} \otimes_{P(z)} W_{2}$ the following way: for any $p \in P$, consider the 
map

$$
\begin{aligned}
\operatorname{Id}_{W_{1}} \otimes p: W_{1} \otimes W_{2} & \rightarrow W_{1} \otimes W_{2} \\
w_{1} \otimes w_{2} & \mapsto w_{1} \otimes\left(w_{2} p\right) ;
\end{aligned}
$$

then, by Proposition [1.9, there exists a unique $V$-homomorphism $\operatorname{Id}_{W_{1}} \bigotimes_{P(z)} p$ of $W_{1} \bigotimes_{P(z)} W_{2}$ such that

$$
\bigotimes_{P(z)} \circ\left(\operatorname{Id}_{W_{1}} \otimes p\right)=\overline{\left(\operatorname{Id}_{W_{1}} \bigotimes_{P(z)} p\right)} \circ \bigotimes_{P(z)} .
$$

so we let $p$ act on $W_{1} \bigotimes_{P(z)} W_{2}$ by

$$
w p=\left(\operatorname{Id}_{W_{1}} \bigotimes_{P(z)} p\right)(w)
$$

for any $w \in W_{1} \otimes_{P(z)} W_{2}$, and extend it to the formal completion $\overline{W_{1} \otimes_{P(z)} W_{2}}$; thus (1.16) becomes

$$
\bigotimes_{P(z)}\left(w_{1} \otimes\left(w_{2} p\right)\right)=\bigotimes_{P(z)}\left(w_{1} \otimes w_{2}\right) p
$$

for all $w_{1} \in W_{1}$ and $w_{2} \in W_{2}$. Thus, $W_{1} \bigotimes_{P(z)} W_{2}$ can be seen naturally as a $V$-P-bimodule; moreover, the intertwining map $\bigotimes_{P(z)}$ commutes with the action of $P$. In particular, $P$ commutes with the intertwining operator $\mathcal{Y}_{\bigotimes_{P(z)}, 0}$.

Proposition 1.30. Let $\mathcal{Y}_{1} \in\left(\begin{array}{c}W_{4} \\ W_{1} M\end{array}\right)$ and $\mathcal{Y}_{2} \in\left(\begin{array}{c}M \\ W_{2} W_{3}\end{array}\right)$ be two logarithmic intertwining operators, where $W_{1}, W_{2}, W_{3}$ are $V$-P-bimodules, $M$ is a grading-restricted generalized $V$-module, and $P$ commutes with $\mathcal{Y}_{1}$ and $\mathcal{Y}_{2}$. Let $\mathcal{Y}^{1} \in\left(\begin{array}{c}W_{4} \\ W W_{3}\end{array}\right)$ be the logarithmic intertwining operator as in Proposition 1.10 (1.) (here $\left.W=W_{1} \otimes_{P\left(z_{0}\right)} W_{2}\right)$. Then $P$ commutes with $\mathcal{Y}^{1}$.

Proof. Let $p$ be any element in $P$; for any two $V$-P-bimodules $W^{2}, W^{3}$, grading-restricted generalized $V$-module $W^{1}$, and logarithmic intertwining operator $\mathcal{Y} \in\left(\begin{array}{c}W^{3} \\ W^{1} W^{2}\end{array}\right)$, consider the logarithmic intertwining operators $\mathcal{Y}_{p}, \mathcal{Y}^{p} \in\left(\begin{array}{c}W^{3} \\ W^{1} W^{2}\end{array}\right)$ defined by

$$
\begin{aligned}
& \mathcal{Y}_{p}\left(w^{(1)}, x\right) w^{(2)}=\mathcal{Y}\left(w^{(1)}, x\right)\left(w^{(2)} p\right) \\
& \mathcal{Y}^{p}\left(w^{(1)}, x\right) w^{(2)}=\left(\mathcal{Y}\left(w^{(1)}, x\right) w^{(2)}\right) p
\end{aligned}
$$

for all $w^{(1)} \in W^{1}, w^{(2)} \in W^{2}$. It is clear that $\mathcal{Y}_{p}$ (resp. $\mathcal{Y}^{p}$ ) is a logarithmic intertwining operator since $p$ acts on $W^{2}$ (resp. $W^{3}$ ) as a $V$-module homomorphism. Then $P$ commutes with $\mathcal{Y}$ if and only if $\mathcal{Y}_{p}=\mathcal{Y}^{p}$ for all $p \in P$. Consider $\left(\mathcal{Y}^{1}\right)_{p}$ and $\left(\mathcal{Y}^{1}\right)^{p}$ : then for $w_{(1)} \in W_{1}$, $w_{(2)} \in W_{2}, w_{(3)} \in W_{3}, w_{(4)}^{\prime} \in W_{4}^{\prime}$, and for all $z_{0}, z_{1}, z_{2}$ such that $z_{0}=z_{1}-z_{2},\left|z_{1}\right|>\left|z_{2}\right|>$ $\left|z_{0}\right|>0$,

$$
\begin{aligned}
\left\langle w_{(4)}^{\prime},\right. & \left.,\left(\mathcal{Y}^{1}\right)^{p}\left(\mathcal{Y}_{\bigotimes_{P\left(z_{0}\right)}, 0}\left(w_{(1)}, z_{0}\right) w_{(2)}, z_{2}\right) w_{(3)}\right\rangle \\
& =\left\langle w_{(4)}^{\prime},\left(\mathcal{Y}^{1}\left(\mathcal{Y}_{\bigotimes_{P\left(z_{0}\right)}, 0}\left(w_{(1)}, z_{0}\right) w_{(2)}, z_{2}\right) w_{(3)}\right) p\right\rangle \\
& =\left\langle p w_{(4)}^{\prime}, \mathcal{Y}^{1}\left(\mathcal{Y}_{\varpi_{P\left(z_{0}\right)}, 0}\left(w_{(1)}, z_{0}\right) w_{(2)}, z_{2}\right) w_{(3)}\right\rangle \\
& =\left\langle p w_{(4)}^{\prime}, \mathcal{Y}_{1}\left(w_{(1)}, z_{1}\right) \mathcal{Y}_{2}\left(w_{(2)}, z_{2}\right) w_{(3)}\right\rangle \\
& =\left\langle w_{(4)}^{\prime},\left(\mathcal{Y}_{1}\left(w_{(1)}, z_{1}\right) \mathcal{Y}_{2}\left(w_{(2)}, z_{2}\right) w_{(3)}\right) p\right\rangle
\end{aligned}
$$




$$
\begin{aligned}
& =\left\langle w_{(4)}^{\prime}, \mathcal{Y}_{1}\left(w_{(1)}, z_{1}\right)\left(\mathcal{Y}_{2}\left(w_{(2)}, z_{2}\right) w_{(3)}\right) p\right\rangle \\
& =\left\langle w_{(4)}^{\prime}, \mathcal{Y}_{1}\left(w_{(1)}, z_{1}\right)\left(\mathcal{Y}_{2}\right)^{p}\left(w_{(2)}, z_{2}\right) w_{(3)}\right\rangle
\end{aligned}
$$

Similarly,

$$
\begin{aligned}
\left\langle w_{(4)}^{\prime},\right. & \left.\left(\mathcal{Y}^{1}\right)_{p}\left(\mathcal{Y}_{\varpi_{P\left(z_{0}\right), 0}}\left(w_{(1)}, z_{0}\right) w_{(2)}, z_{2}\right) w_{(3)}\right\rangle \\
& =\left\langle w_{(4)}^{\prime}, \mathcal{Y}^{1}\left(\mathcal{Y}_{\varpi_{P\left(z_{0}\right)}, 0}\left(w_{(1)}, z_{0}\right) w_{(2)}, z_{2}\right)\left(w_{(3)} p\right)\right\rangle \\
& =\left\langle w_{(4)}^{\prime}, \mathcal{Y}_{1}\left(w_{(1)}, z_{1}\right) \mathcal{Y}_{2}\left(w_{(2)}, z_{2}\right)\left(w_{(3)} p\right)\right\rangle \\
& =\left\langle w_{(4)}^{\prime}, \mathcal{Y}_{1}\left(w_{(1)}, z_{1}\right)\left(\mathcal{Y}_{2}\right)_{p}\left(w_{(2)}, z_{2}\right) w_{(3)}\right\rangle \\
& =\left\langle w_{(4)}^{\prime}, \mathcal{Y}_{1}\left(w_{(1)}, z_{1}\right)\left(\mathcal{Y}_{2}\right)^{p}\left(w_{(2)}, z_{2}\right) w_{(3)}\right\rangle .
\end{aligned}
$$

Thus, by uniqueness in Proposition 1.10 (2.) applied to the logarithmic intertwining operators $\mathcal{Y}_{1}$ and $\left(\mathcal{Y}_{2}\right)^{p}$ we see that $\left(\mathcal{Y}^{1}\right)^{p}=\left(\mathcal{Y}^{1}\right)_{p}$ and therefore $P$ commutes with $\mathcal{Y}^{1}$.

Proposition 1.31. Using the notation of Proposition 1.10 (2.), suppose the modules $W_{3}$ and $W_{4}$ are $V$-P-bimodules, and the logarithmic intertwining operator $\mathcal{Y}^{1}$ commutes with $P$. Then the logarithmic intertwining operator $\mathcal{Y}_{1}$ also commutes with $P$.

Proof. Note that since $W_{3}$ is a $V$-P-bimodule, the right action of $P$ on $W_{3}$ defines a right action of $P$ on $W_{2} \bigotimes_{P(z)} W_{3}$, and $\mathcal{Y}_{\bigotimes_{P\left(z_{2}\right)}, 0}$ commutes with $P$. Now for $w_{(1)} \in W_{1}, w_{(2)} \in W_{2}$, $w_{(3)} \in W_{3}$ and $w_{(4)}^{\prime} \in W_{4}^{\prime}$, and for complex numbers $z_{0}, z_{1}, z_{2}$ such that $\left|z_{1}\right|>\left|z_{2}\right|>\left|z_{0}\right|>0$, $z_{0}=z_{1}-z_{2}$, for any $p \in P$

$$
\begin{aligned}
\left\langle w_{(4)}^{\prime},\right. & \left.\left(\mathcal{Y}_{1}\right)^{p}\left(w_{(1)}, z_{1}\right) \mathcal{Y}_{\bigotimes_{P\left(z_{2}\right)}, 0}\left(w_{(2)}, z_{2}\right) w_{(3)}\right\rangle \\
& =\left\langle w_{(4)}^{\prime},\left(\mathcal{Y}_{1}\left(w_{(1)}, z_{1}\right) \mathcal{Y}_{\bigotimes_{P\left(z_{2}\right)}, 0}\left(w_{(2)}, z_{2}\right) w_{(3)}\right) p\right\rangle \\
& =\left\langle p w_{(4)}^{\prime}, \mathcal{Y}_{1}\left(w_{(1)}, z_{1}\right) \mathcal{Y}_{\bigotimes_{P\left(z_{2}\right)}, 0}\left(w_{(2)}, z_{2}\right) w_{(3)}\right\rangle \\
& =\left\langle p w_{(4)}^{\prime}, \mathcal{Y}^{1}\left(\mathcal{Y}^{2}\left(w_{(1)}, z_{0}\right) w_{(2)}, z_{2}\right) w_{(3)}\right\rangle \\
& =\left\langle w_{(4)}^{\prime},\left(\mathcal{Y}^{1}\left(\mathcal{Y}^{2}\left(w_{(1)}, z_{0}\right) w_{(2)}, z_{2}\right) w_{(3)}\right) p\right\rangle \\
& =\left\langle w_{(4)}^{\prime},\left(\mathcal{Y}^{1}\right)^{p}\left(\mathcal{Y}^{2}\left(w_{(1)}, z_{0}\right) w_{(2)}, z_{2}\right) w_{(3)}\right\rangle .
\end{aligned}
$$

Similarly, since $\mathcal{Y}_{\bigotimes_{P\left(z_{2}\right)}, 0}$ commutes with $P$,

$$
\begin{aligned}
\left\langle w_{(4)}^{\prime},\right. & \left.\left(\mathcal{Y}_{1}\right)_{p}\left(w_{(1)}, z_{1}\right) \mathcal{Y}_{\bigotimes_{P\left(z_{2}\right)}, 0}\left(w_{(2)}, z_{2}\right) w_{(3)}\right\rangle \\
& =\left\langle w_{(4)}^{\prime}, \mathcal{Y}_{1}\left(w_{(1)}, z_{1}\right)\left(\mathcal{Y}_{\bigotimes_{P\left(z_{2}\right)}, 0}\left(w_{(2)}, z_{2}\right) w_{(3)}\right) p\right\rangle \\
& =\left\langle w_{(4)}^{\prime}, \mathcal{Y}_{1}\left(w_{(1)}, z_{1}\right) \mathcal{Y}_{\bigotimes_{P\left(z_{2}\right)}, 0}\left(w_{(2)}, z_{2}\right)\left(w_{(3)} p\right)\right\rangle \\
& =\left\langle w_{(4)}^{\prime}, \mathcal{Y}^{1}\left(\mathcal{Y}^{2}\left(w_{(1)}, z_{0}\right) w_{(2)}, z_{2}\right)\left(w_{(3)} p\right)\right\rangle \\
& =\left\langle w_{(4)}^{\prime},\left(\mathcal{Y}^{1}\right)_{p}\left(\mathcal{Y}^{2}\left(w_{(1)}, z_{0}\right) w_{(2)}, z_{2}\right) w_{(3)}\right\rangle \\
& =\left\langle w_{(4)}^{\prime},\left(\mathcal{Y}^{1}\right)^{p}\left(\mathcal{Y}^{2}\left(w_{(1)}, z_{0}\right) w_{(2)}, z_{2}\right) w_{(3)}\right\rangle .
\end{aligned}
$$


The conclusion thus follows from uniqueness in Proposition 1.10 part (2.) applied to the logarithmic intertwining operators $\left(\mathcal{Y}^{1}\right)^{p}$ and $\mathcal{Y}^{2}$.

We summarize these results in the following.

Theorem 1.32 (Associativity for $P$-intertwining operators). Let $W_{1}, W_{2}, W_{3}$ be $V$-P-bimodules.

(i) Let $M$ be a $V$-P-bimodule and $\mathcal{Y}_{1}, \mathcal{Y}_{2}$ be two P-intertwining operators of types $\left(\begin{array}{c}W_{4} \\ W_{1} M\end{array}\right)$, $\left(\begin{array}{c}M \\ W_{2} W_{3}\end{array}\right)$, respectively. Then there exist a grading-restricted generalized module $W$, a $P$ intertwining operator $\mathcal{Y}^{1}$ of type $\left(\begin{array}{c}W_{4} \\ W W_{3}\end{array}\right)$ and a logarithmic intertwining operator $\mathcal{Y}^{2}$ of type $\left(\begin{array}{c}W \\ W_{1} W_{2}\end{array}\right)$ such that for all $w_{(1)} \in W_{1}, w_{(2)} \in W_{2}, w_{(3)} \in W_{3}, w_{(4)} \in W_{4}^{\prime}$,

$$
\begin{aligned}
\left\langle w_{(4)}^{\prime},\right. & \left.\mathcal{Y}_{1}\left(w_{(1)}, z_{1}\right) \mathcal{Y}_{2}\left(w_{(2)}, z_{2}\right) w_{(3)}\right\rangle \\
= & \left\langle w_{(4)}^{\prime}, \mathcal{Y}^{1}\left(\mathcal{Y}^{2}\left(w_{(1)}, z_{1}-z_{2}\right) w_{(2)}, z_{2}\right) w_{(3)}\right\rangle
\end{aligned}
$$

for all $z_{1}, z_{2}$ such that $\left|z_{1}\right|>\left|z_{2}\right|>\left|z_{1}-z_{2}\right|$.

(ii) Let $W$ be a grading-restricted generalized $V$-module, $\mathcal{Y}^{1}$ a logarithmic intertwining operator of type $\left(\begin{array}{c}W_{4} \\ W W_{3}\end{array}\right)$, and $\mathcal{Y}^{2}$ a P-intertwining operator of type $\left(\begin{array}{c}W \\ W_{1} W_{2}\end{array}\right)$. Then there exists a $V$-P-bimodule $M$ and P-intertwining operators $\mathcal{Y}_{1}, \mathcal{Y}_{2}$ of types $\left(\begin{array}{c}W_{4} \\ W_{1} M\end{array}\right),\left(\begin{array}{c}M \\ W_{2} W_{3}\end{array}\right)$, respectively such that the same conclusion as in (i) holds.

We now state the commutativity property.

Theorem 1.33 (Commutativity for $P$-intertwining operators). Let $\mathcal{Y}_{1}, \mathcal{Y}_{2}$ be $P$-intertwining operators of types $\left(\begin{array}{c}W_{4} \\ W_{1} M\end{array}\right),\left(\begin{array}{c}M \\ W_{2} W_{3}\end{array}\right)$, respectively, for grading-restricted generalized $V$-modules $W_{1}, W_{2}$ and $V$-P-bimodules $W_{3}, W_{4}, M$. Then there exist a $V$-P-bimodule $M_{1}$ and logarithmic intertwining operators $\mathcal{Y}_{3}, \mathcal{Y}_{4}$ of type $\left(\begin{array}{c}W_{4} \\ W_{2} M_{1}\end{array}\right),\left(\begin{array}{c}M_{1} \\ W_{1} W_{3}\end{array}\right)$ respectively, commuting with the action of $P$, such that for any $w_{(1)} \in W_{1}, w_{(2)} \in W_{2}, w_{(3)} \in W_{3}, w_{(4)}^{\prime} \in W_{4}^{\prime}$, the multivalued analytic function

$$
\left\langle w_{(4)}^{\prime}, \mathcal{Y}_{1}\left(w_{(1)}, z_{1}\right) \mathcal{Y}_{2}\left(w_{(2)}, z_{2}\right) w_{(3)}\right\rangle
$$

on the region $\left|z_{1}\right|>\left|z_{2}\right|>0$ and the multivalued analytic function

$$
\left\langle w_{(4)}^{\prime}, \mathcal{Y}_{3}\left(w_{(2)}, z_{2}\right) \mathcal{Y}_{4}\left(w_{(1)}, z_{1}\right) w_{(3)}\right\rangle
$$

on the region $\left|z_{2}\right|>\left|z_{1}\right|>0$ are analytic extensions of each other.

Proof. Using associativity twice, by the previous propositions one sees that all the outer intertwining operators commute with $P$. Recall ([HLZ2]) the operator $\Omega_{r}: \mathcal{V}_{W^{1} W^{2}}^{W^{3}} \rightarrow \mathcal{V}_{W^{2} W^{1}}^{W^{3}}$ defined by

$$
\Omega_{r}(\mathcal{Y})\left(w_{(2)}, x\right) w_{(1)}=e^{x L(-1)} \mathcal{Y}\left(w_{(1)}, e^{(2 r+1) \pi i} x\right) w_{(2)}
$$

for any $r \in \mathbb{Z}$; then $\Omega_{-r-1}\left(\Omega_{r}(\mathcal{Y})\right)=\Omega_{r}\left(\Omega_{-r-1}(\mathcal{Y})\right)=\mathcal{Y}$ for any $\mathcal{Y} \in \mathcal{V}_{W^{1} W^{2}}^{W^{3}}$. 
We know by Theorem 1.32 that there exist a module $M$, an intertwining operator $\mathcal{Y}^{1}$ and a $P$-intertwining operator $\mathcal{Y}^{2}$ such that

$$
\begin{aligned}
& \left\langle w_{(4)}^{\prime}, \mathcal{Y}_{1}\left(w_{(1)}, z_{1}\right) \mathcal{Y}_{2}\left(w_{(2)}, z_{2}\right) w_{(3)}\right\rangle \\
& \quad=\left\langle w_{(4)}^{\prime}, \mathcal{Y}^{2}\left(\mathcal{Y}^{1}\left(w_{(1)}, z_{0}\right) w_{(2)}, z_{2}\right) w_{(3)}\right\rangle
\end{aligned}
$$

now substituting

$$
\begin{aligned}
\mathcal{Y}^{1}\left(w_{(1)}, x_{0}\right) w_{(2)} & =\Omega_{0}\left(\Omega_{-1}\left(\mathcal{Y}^{1}\right)\right)\left(w_{(1)}, x_{0}\right) w_{(2)} \\
& =e^{x_{0} L(-1)} \Omega_{-1}\left(\mathcal{Y}^{1}\right)\left(w_{(2)}, e^{\pi i} x_{0}\right) w_{(1)}
\end{aligned}
$$

we obtain (in the region $\left|z_{1}\right|>\left|z_{2}\right|>\left|z_{1}-z_{2}\right|$ )

$$
\begin{aligned}
& \left\langle w_{(4)}^{\prime}, \mathcal{Y}_{1}\left(w_{(1)}, z_{1}\right) \mathcal{Y}_{2}\left(w_{(2)}, z_{2}\right) w_{(3)}\right\rangle \\
& \quad=\left\langle w_{(4)}^{\prime}, \mathcal{Y}^{2}\left(\Omega_{1}\left(\mathcal{Y}^{1}\right)\left(w_{(2)}, e^{\pi i} z_{0}\right) w_{(1)}, z_{2}+z_{0}\right) w_{(3)}\right\rangle ;
\end{aligned}
$$

which is an extension of $\left\langle w_{(4)}^{\prime}, \mathcal{Y}^{2}\left(\Omega_{1}\left(\mathcal{Y}^{1}\right)\left(w_{(2)}, z_{2}-z_{1}\right) w_{(1)}, z_{1}\right) w_{(3)}\right\rangle$ defined on the region $\left|z_{1}\right|>\left|z_{2}-z_{1}\right|>0$. Now by Theorem 1.32 (ii), we know that there exist a $V$-P-bimodule $M_{1}$ and $P$ intertwining operators $\mathcal{Y}_{3}, \mathcal{Y}_{4}$ of type $\left(\begin{array}{c}W_{4} \\ W_{2} M_{1}\end{array}\right),\left(\begin{array}{c}M_{1} \\ W_{1} W_{3}\end{array}\right)$ respectively, such that

$$
\begin{gathered}
\left\langle w_{(4)}^{\prime}, \mathcal{Y}^{2}\left(\Omega_{1}\left(\mathcal{Y}^{1}\right)\left(w_{(2)}, z_{2}-z_{1}\right) w_{(1)}, z_{1}\right) w_{(3)}\right\rangle \\
=\left\langle w_{(4)}^{\prime}, \mathcal{Y}_{3}\left(w_{(2)}, z_{2}\right) \mathcal{Y}_{4}\left(w_{(1)}, z_{1}\right) w_{(3)}\right\rangle
\end{gathered}
$$

This concludes the proof.

\section{Genus-one correlation functions}

\subsection{Differential Equations}

In this section we derive a system of differential equations satisfied by the formal $q$-traces, using the identities obtained in the previous section. The main technical assumption used in this section is the $C_{2}$-cofiniteness of $V$, which is used to prove that certain particular modules over a ring of functions are finitely generated.

In this section, we assume that the vertex operator algebra $V$ is $C_{2}$-cofinite. Then by Lemma 2.11 and Proposition 4.3 in [H6], every grading-restricted generalized $V$-module is $C_{2}$-cofinite and is $\mathbb{R}$-graded. We denote by $G$ the space of all multivalued analytic functions defined on the region $\left|z_{1}\right|>\left|z_{2}\right|>\ldots>\left|z_{n}\right|>0$ with preferred branches on $\left|z_{1}\right|>\left|z_{2}\right|>$ $\ldots>\left|z_{n}\right|>0,0 \leq \arg z_{i}<2 \pi$ for $i=1 \ldots n$. For any such function $f\left(z_{1}, \ldots, z_{n}\right)$, the function $f\left(q_{z_{1}}, \ldots, q_{z_{n}}\right)$ is a multivalued analytic function defined when $\left|q_{z_{1}}\right|>\ldots>\left|q_{z_{n}}\right|$. We denote by $G_{q}$ the space of such functions. Let

$$
R=\mathbb{C}\left[\tilde{G}_{4}(q), \tilde{G}_{6}(q), \tilde{\wp}_{2}\left(z_{i}-z_{j} ; q\right), \tilde{\wp}_{3}\left(z_{i}-z_{j} ; q\right)\right] .
$$


For $V$-modules $W_{1}, W_{1}, \ldots, W_{n}$ we denote by $T$ the graded $R$-module

$$
R \otimes W_{1} \otimes \ldots \otimes W_{n},
$$

with grading induced by the grading by generalized eigenvalues on $W_{1}, \ldots, W_{n}$. Denote by $T_{r}$ the homogeneous subspace of degree $r \in \mathbb{R}$; moreover, define a filtration on $T$ by $F(T)_{r}=\coprod_{s \leq r} T_{s}$. Let $J$ be the $R$-submodule of $T$ generated by the elements

$$
\begin{aligned}
& A_{j}\left(v ; w_{1}, \ldots, w_{n}\right) \\
& \quad=1 \otimes w_{1} \otimes \ldots \otimes w_{j-1} \otimes v_{-2} w_{j} \otimes w_{j+1} \otimes \ldots \otimes w_{n} \\
& \quad+\sum_{k=1}^{\infty}(2 k+1) \tilde{G}_{2 k+2}(q) \otimes w_{1} \otimes \ldots \otimes w_{j-1} \otimes v_{2 k} w_{j} \otimes w_{j+1} \otimes \ldots \otimes w_{n} \\
& \quad+\sum_{i \neq j} \sum_{m=0}^{\infty}(-1)^{m}(m+1) \tilde{\wp}_{m+2}\left(z_{i}-z_{j}\right) \otimes \\
& \quad w_{1} \otimes \ldots \otimes w_{j-1} \otimes v_{m} w_{j} \otimes w_{j+1} \otimes \ldots \otimes w_{n}
\end{aligned}
$$

for $v \in V, w_{i} \in W_{i}, i=1 \ldots, n$, and $1 \leq j \leq n$. The filtration on $T$ induces one on $J$, and we will denote by $F(J)_{r}$ the $r$-th subspace in the filtration.

Proposition 2.1. There exists $N \in \mathbb{R}$ such that for any $r \in \mathbb{R}, F(T)_{r}=F(T)_{N}+F(J)_{r}$.

Proof. Let $N$ such that

$$
\coprod_{r>N} T_{r} \subseteq \sum_{i=1}^{n} R \otimes W_{1} \otimes \ldots W_{i-1} \otimes C_{2}\left(W_{i}\right) \otimes W_{i+1} \otimes \ldots W_{n} ;
$$

then clearly if $r \leq N, F(T)_{r} \subseteq F(T)_{N}=F(T)_{N}+F(J)_{r}$. We now prove on induction on $k \in \mathbb{N}$ that if $r=N+k$, then $F(T)_{r}=F(T)_{n}+F(J)_{r}$. Let $r=N+k+1$ and let $t \in F(T)_{r}$. By definition of $N$, we can assume

$$
t=1 \otimes w_{1} \otimes \cdots \otimes w_{i-1} \otimes v_{-2} w_{i} \otimes w_{i+1} \otimes \ldots \otimes w_{n}
$$

for some $v \in V$ and $w_{j} \in W_{j}, j=1 \ldots, n$. Observe that the element $A\left(v ; w_{1}, \ldots, w_{n}\right)$ belongs to $F(J)_{r}$ and

$$
\begin{gathered}
S=\sum_{k=1}^{\infty}(2 k+1) \tilde{G}_{2 k+2}(q) \otimes w_{1} \otimes \ldots \otimes w_{j-1} \otimes v_{2 k} w_{j} \otimes w_{j+1} \otimes \ldots \otimes w_{n} \\
\quad+\sum_{i \neq j} \sum_{m=0}^{\infty}(-1)^{m}(m+1) \tilde{\wp}_{m+2}\left(z_{i}-z_{j}\right) \otimes \\
\quad w_{1} \otimes \ldots \otimes w_{j-1} \otimes v_{m} w_{j} \otimes w_{j+1} \otimes \ldots \otimes w_{n}
\end{gathered}
$$

belongs to $F(T)_{r-1}$. By induction hypothesis, $S \in F(T)_{N}+F(J)_{r-1}$. Then $t=A\left(v, w_{1}, \ldots, w_{n}\right)-$ $s \in F(J)_{r}+F(T)_{N}+F(J)_{r-1}=F(T)_{N}+F(J)_{r}$. This concludes the proof. 
Corollary 2.2. We have $T=F(T)_{N}+J$ and $T / J$ is a finitely generated $R$-module.

Proof. The first assertion follows immediately from Proposition 2.1; the second follows since $F(T)_{N}$ is finitely generated.

In the following we will fix a finite-dimensional associative algebra $P$ and a symmetric linear function $\phi$ on $P$. Recall that the cenrtal charge of $V$ is $c$. For $V$-P-bimodules $\tilde{W}_{0} \ldots \tilde{W}_{n}$ with $\tilde{W}_{0}=\tilde{W}_{n}$ and $P$-intertwining operators $\mathcal{Y}_{i}$ of type $\left(\begin{array}{c}\tilde{W}_{i-1} \\ W_{i} \tilde{W}_{i}\end{array}\right), i=1 \ldots n$, and for $w_{i} \in W_{i}$, $i=1, \ldots, n$, we consider the map

$$
\begin{aligned}
& F_{\mathcal{Y}_{1}, \ldots, \mathcal{Y}_{n}}\left(w_{1}, \ldots, w_{n} ; z_{1}, \ldots, z_{n} ; q\right) \\
& \quad=\operatorname{tr}_{\tilde{W}_{n}}^{\phi} \mathcal{Y}_{1}\left(\mathcal{U}\left(q_{z_{1}}\right) w_{1}, q_{z_{1}}\right) \cdots \mathcal{Y}_{n}\left(\mathcal{U}\left(q_{z_{n}}\right) w_{n}, q_{z_{n}}\right) q^{L(0)-\frac{c}{24}}
\end{aligned}
$$

and we extend it to an $R$-module map $\psi_{\mathcal{Y}_{1}, \ldots, \mathcal{Y}_{n}}: T \rightarrow G_{q}((q))[\log q]$ defined by

$$
f \otimes w_{1} \otimes \ldots \otimes w_{n} \mapsto f \cdot F\left(w_{1}, \ldots, w_{1} ; z_{1}, \ldots, z_{n} ; q\right)
$$

We will also use the notation $F_{\mathcal{Y}_{1}, \ldots, \mathcal{Y}_{n}}^{\phi}$ and $\psi_{\mathcal{Y}_{1}, \ldots, \mathcal{Y}_{n}}^{\phi}$ when we need to specify the dependence on the symmetric function $\phi$.

Proposition 2.3. The submodule $J$ is contained in the kernel of $\psi_{\mathcal{Y}_{1}, \ldots, \mathcal{Y}_{n}}$; hence $\psi_{\mathcal{Y}_{1}, \ldots, \mathcal{Y}_{n}}$ induces a map, also denoted by $\psi_{\mathcal{Y}_{1}, \ldots, \mathcal{Y}_{n}}$, from $T / J$ to $G_{q}((q))[\log q]$.

Proof. This follows from applying $\psi_{\mathcal{Y}_{1}, \ldots, \mathcal{Y}_{n}}$ to (1.14) with $l=2$; the resulting equation implies $\psi_{\mathcal{Y}_{1}, \ldots, \mathcal{Y}_{n}}\left(A_{j}\left(v ; w_{1}, \ldots, w_{n}\right)\right)=0$ for all $v \in V, w_{1} \in W_{1}, \ldots, w_{n} \in W_{n}$.

Proposition 2.4. For any $w_{1} \in W_{1}, \ldots w_{n} \in W_{n}$, we have the $L(-1)$-derivative property

$$
\begin{aligned}
& \frac{\partial}{\partial z_{i}} F_{\mathcal{Y}_{1}, \ldots, \mathcal{Y}_{n}}\left(w_{1}, \ldots, w_{n} ; z_{1}, \ldots, z_{n} ; q\right) \\
& \quad=F_{\mathcal{Y}_{1}, \ldots, \mathcal{Y}_{n}}\left(w_{1}, \ldots, w_{j-1}, L(-1) w_{j}, w_{j+1}, \ldots, w_{n} ; z_{1}, \ldots, z_{n} ; q\right)
\end{aligned}
$$

Proof. This is an immediate consequence of Lemma 1.7.

Proposition 2.5. Consider grading-restricted generalized $V$-modules $W_{i}, \tilde{W}_{i}$ for $i=1, \ldots, n$, with $\tilde{W}_{0}=\tilde{W}_{n}$, and logarithmic intertwining operators $\mathcal{Y}_{i}$ of type $\left(\begin{array}{c}\tilde{W}_{i-1} \\ W_{i} \tilde{W}_{i}\end{array}\right)$ for $i=1, \ldots, n$. Moreover, suppose $\tilde{W}_{0}$ is a V-P-bimodule projective as a right P-module. Then for any homogeneous elements $w_{1} \in W_{1}, \ldots, w_{n} \in W_{n}$, and any $j=1, \ldots, n$, we have

$$
\begin{aligned}
\left((2 \pi i)^{2} q\right. & \left.\frac{\partial}{\partial q}+\tilde{G}_{2}(q) \sum_{i=1}^{n} \text { wt } w_{i}+\tilde{G}_{2}(q) \sum_{i=1}^{n} z_{i} \frac{\partial}{\partial z_{i}}-\sum_{i \neq j} \tilde{\wp}_{1}\left(z_{i}-z_{j} ; q\right) \frac{\partial}{\partial z_{i}}\right) . \\
& \cdot F_{\mathcal{Y}_{1}, \ldots, \mathcal{Y}_{n}}\left(w_{1}, \ldots, w_{n} ; z_{1}, \ldots, z_{n} ; q\right)
\end{aligned}
$$




$$
\begin{aligned}
& +\tilde{G}_{2}(q) \sum_{i=1}^{n} F_{\mathcal{Y}_{2}, \ldots, \mathcal{Y}_{n}}\left(w_{1}, \ldots, w_{i-1}, L(0)_{n} w_{i}, w_{i+1}, \ldots, w_{n} ; z_{1}, \ldots, z_{n} ; q\right) \\
& =F_{\mathcal{Y}_{2}, \ldots, \mathcal{Y}_{n}}\left(w_{1}, \ldots, w_{j-1}, L(-2) w_{j}, w_{j+1}, \ldots, w_{n} ; z_{1}, \ldots, z_{n} ; q\right) \\
& -\sum_{k=1}^{\infty} \tilde{G}_{2 k+2}(q) \cdot \\
& \quad \cdot F_{\mathcal{Y}_{1}, \ldots, \mathcal{Y}_{n}}\left(w_{1}, \ldots, w_{j-1}, L(2 k) w_{j}, w_{j+1}, \ldots, w_{n} ; z_{1}, \ldots, z_{n} ; q\right) \\
& \left.+\sum_{i \neq j} \sum_{m=1}^{\infty}(-1)^{m} \tilde{\wp}_{m+1}\left(z_{i}-z_{j}\right) ; q\right) \cdot \\
& \quad \cdot F_{\mathcal{Y}_{1}, \ldots, \mathcal{Y}_{n}}\left(w_{1}, \ldots, w_{i-1}, L(m-1) w_{i}, w_{i+1}, \ldots, w_{n} ; z_{1}, \ldots, z_{n} ; q\right)
\end{aligned}
$$

Proof. This follows from (1.15) and the definition of $\psi_{\mathcal{Y}_{1}, \ldots, \mathcal{Y}_{n}}$, using Lemma 1.6 and (2.18).

Remark 2.6. This formula differs from the one obtained in [H2], by the presence of the additional term

$$
G_{2}(q) \sum_{i=1}^{n} F_{\mathcal{Y}_{2}, \ldots, \mathcal{Y}_{n}}\left(w_{1}, \ldots, w_{i-1}, L(0)_{n} w_{i}, w_{i+1}, \ldots, w_{n} ; z_{1}, \ldots, z_{n} ; q\right)
$$

due to the non-semisimplicity of the operator $L(0)$.

We will now consider a second grading on the space $T$ by "modular weights": define the modular weight on the ring $R$ by assigning weight $2 k$ to the function $G_{2 k}(q)$ and weight $m$ to $\tilde{\wp}_{m}(z ; q)$, and denote by $R_{p}$ the homogeneous subspace of modular weight $p$. Now if $t=f \otimes w_{1} \otimes \ldots \otimes w_{n} \in T$, with homogeneous $f \in R_{p}$ and $w_{i} \in W_{i}, i=1, \ldots, n$, we assign $t$ modular weight $p+\sum_{i=1}^{n}$ wt $w_{i}$. Clearly from this definition, if $v \in V$ and $w_{i} \in W_{i}$, $i=1, \ldots, n$ are homogeneous, then the element $A\left(v ; w_{1}, \ldots, w_{n}\right) \in J$ has modular weight wt $v+\sum_{i=1}^{n}$ wt $w_{i}+1$. As a consequence, we have

Proposition 2.7. The ideal $J$ is graded by modular weights; in particular, this grading induces a grading on the quotient module $T / J$.

Proposition 2.8. Let $W_{1}, \ldots W_{n}$ be grading-restricted generalized modules for the vertex operator algebra $V$, and consider homogeneous $w_{i} \in W_{i}$ for $i=1, \ldots n$. Then there exist elements $a_{p, i} \in R_{p}$ for $p=1, \ldots, m$ such that for any $V$-P-bimodules $\tilde{W}_{j}$ and $P$-intertwining operators $\mathcal{Y}_{j}$ of type $\left(\begin{array}{c}\tilde{W}_{j-1} \\ W_{j} \tilde{W}_{j}\end{array}\right), j=1, \ldots, n$, with $\tilde{W}_{0}=\tilde{W}_{n}$ projective as a right $P$-module, the series

$$
\operatorname{tr}_{\tilde{W}_{n}}^{\phi} \mathcal{Y}_{1}\left(\mathcal{U}\left(q_{z_{1}}\right) w_{1}, q_{z_{1}}\right) \cdot \ldots \cdot \mathcal{Y}_{n}\left(\mathcal{U}\left(q_{z_{n}}\right) w_{n}, q_{z_{n}}\right) q^{L(0)-\frac{c}{24}}
$$

satisfies the differential equations

$$
\frac{\partial^{m}}{\partial z_{i}^{m}} \varphi+\sum_{p=1}^{m} a_{p, i}\left(z_{1}, \ldots, z_{n} ; q\right) \frac{\partial^{m-p}}{\partial z_{i}^{m-p}} \varphi=0 .
$$


for $i=1, \ldots, n$, in the region $1>\left|q_{z_{1}}\right|>\ldots>\left|q_{z_{1}}\right|>|q|>0$.

Proof. Consider the submodule $M_{i}$ of $T / J$ generated by the elements

$$
1 \otimes w_{1} \otimes \ldots \otimes w_{i-1} \otimes L(-1)^{k} w_{i} \otimes w_{i+1} \otimes \ldots w_{n}+J
$$

for $k \in \mathbb{N}$. Since $R$ is Noetherian and $T / J$ is finitely generated, $M_{i}$ is also finitely generated. Therefore there exists an integer $m$ and elements $a_{p, i} \in R$ such that

$$
\begin{aligned}
& 1 \otimes w_{1} \otimes \ldots \otimes w_{i-1} \otimes L(-1)^{m} w_{i} \otimes w_{i+1} \otimes \ldots w_{n}+J \\
& =\sum_{i=1}^{m} a_{p, i}\left(z_{1}, \ldots, z_{n} ; q\right) . \\
& \quad \cdot 1 \otimes w_{1} \otimes \ldots \otimes w_{i-1} \otimes L(-1)^{m-p} w_{i} \otimes w_{i+1} \otimes \ldots w_{n}+J .
\end{aligned}
$$

Note that since the modular weight of $1 \otimes w_{1} \otimes \ldots \otimes w_{i-1} \otimes L(-1)^{k} w_{i} \otimes w_{i+1} \otimes \ldots w_{n}+J$ is $\sum_{i=1}^{n}$ wt $w_{i}+k$, we can choose the element $a_{p, i}$ to have degree $p$. The conclusion follows by applying the map $\psi_{\mathcal{Y}_{1}, \ldots, \mathcal{Y}_{n}}$ to both sides and using the $L(-1)$ derivative property.

Given a logarithmic intertwining operator $\mathcal{Y}$ of type $\left(\begin{array}{c}W_{3} \\ W_{1} W_{2}\end{array}\right)$, for grading-restricted generalized modules $W_{1}, W_{2}, W_{3}$, we will consider the map

$$
\begin{aligned}
\mathcal{Y}^{(k)}: W_{1} \otimes W_{2} & \rightarrow W_{3}\{x\}[\log x] \\
w_{1} \otimes w_{2} & \mapsto \mathcal{Y}\left(L(0)_{n}^{k} w_{1}, x\right) w_{2}
\end{aligned}
$$

for $k \in \mathbb{N}$. Since $L(0)_{n}$ acts on $W_{1}$ as a $V$-module endomorphism, $\mathcal{Y}^{(k)}$ is itself an intertwining operator of the same type. Also, for $j=1, \ldots, n$ define

$$
\begin{aligned}
& Q_{j}\left(1 \otimes w_{1} \otimes \ldots \otimes w_{n}\right)= \\
& w_{1} \otimes \ldots \otimes w_{j-1} \otimes L(-2) w_{j} \otimes w_{j+1} \otimes \ldots \otimes w_{n} \\
& -\sum_{k=1}^{\infty} \tilde{G}_{2 k+2}(q) . \\
& \quad \cdot w_{1} \otimes \ldots \otimes w_{j-1} \otimes L(2 k) w_{j} \otimes w_{j+1} \otimes \ldots \otimes w_{n} \\
& +\sum_{i \neq j} \sum_{m=1}^{\infty}(-1)^{m} \tilde{\wp}_{m+1}\left(z_{i}-z_{j} ; q\right) . \\
& \quad \cdot w_{1} \otimes \ldots \otimes w_{i-1} \otimes L(m-1) w_{i} \otimes w_{i+1} \otimes \ldots \otimes w_{n} .
\end{aligned}
$$

Then, according to (2.19), we have

$$
\begin{aligned}
& \psi_{\mathcal{Y}_{1}, \ldots, \mathcal{Y}_{n}}\left(Q_{j}\left(1 \otimes w_{1} \otimes \ldots \otimes w_{n}\right)\right)= \\
& \quad\left((2 \pi i)^{2} q \frac{\partial}{\partial q}+\tilde{G}_{2}(q) \sum_{i=1}^{n} \mathrm{wt} w_{i}+\tilde{G}_{2}(q) \sum_{i=1}^{n} z_{i} \frac{\partial}{\partial z_{i}}-\sum_{i \neq j} \tilde{\wp}_{1}\left(z_{i}-z_{j} ; q\right) \frac{\partial}{\partial z_{i}}\right) .
\end{aligned}
$$




$$
\begin{aligned}
& \cdot F_{\mathcal{Y}_{1}, \ldots, \mathcal{Y}_{n}}\left(w_{1}, \ldots, w_{n} ; z_{1}, \ldots z_{n} ; q\right) \\
+ & \tilde{G}_{2}(q) \sum_{i=1}^{n} f(q) F_{\mathcal{Y}_{1}, \ldots, \mathcal{Y}_{i-1}, \mathcal{Y}_{i}^{(1)}, \mathcal{Y}_{i+1}, \ldots \mathcal{Y}_{n}}\left(w_{1}, \ldots, w_{n} ; z_{1}, \ldots, z_{n} ; q\right) .
\end{aligned}
$$

For $\alpha \in \mathbb{C}$ and $j=1, \ldots, n$, we define the differential operator

$$
\mathcal{O}_{j}(\alpha)=\left((2 \pi i)^{2} q \frac{\partial}{\partial q}+\tilde{G}_{2}(q) \alpha+\tilde{G}_{2}(q) \sum_{i=1}^{n} z_{i} \frac{\partial}{\partial z_{i}}-\sum_{i \neq j} \tilde{\wp}_{1}\left(z_{i}-z_{j} ; q\right) \frac{\partial}{\partial z_{i}}\right)
$$

and introduce the notation

$$
\begin{aligned}
& \mathcal{D}_{j}(\alpha) F_{\mathcal{Y}_{1}, \ldots, \mathcal{Y}_{n}}\left(w_{1}, \ldots, w_{n} ; z_{1}, \ldots, z_{n} ; q\right) \\
& =\mathcal{O}_{j}(\alpha) F_{\mathcal{Y}_{1}, \ldots, \mathcal{Y}_{n}}\left(w_{1}, \ldots, w_{n} ; z_{1}, \ldots, z_{n} ; q\right) \\
& \quad+\tilde{G}_{2}(q) \sum_{i=1}^{n} F_{\mathcal{Y}_{1}, \ldots, \mathcal{Y}_{n}}\left(w_{1}, \ldots, w_{i-1}, L(0)_{n} w_{i}, w_{i+1}, \ldots, w_{n} ; z_{1}, \ldots, z_{n} ; q\right)
\end{aligned}
$$

and inductively

$$
\begin{aligned}
& \left(\prod_{l=1}^{k} \mathcal{D}_{j}\left(\alpha_{l}\right)\right) F_{\mathcal{Y}_{1}, \ldots, \mathcal{Y}_{n}}\left(w_{1}, \ldots, w_{n} ; z_{1}, \ldots, z_{n} ; q\right) \\
& =\mathcal{O}_{j}\left(\alpha_{1}\right)\left(\left(\prod_{l=2}^{k} \mathcal{D}_{j}\left(\alpha_{l}\right)\right) F_{\mathcal{Y}_{1}, \ldots, \mathcal{Y}_{n}}\left(w_{1}, \ldots, w_{n} ; z_{1}, \ldots, z_{n} ; q\right)\right) \\
& \quad+\tilde{G}_{2}(q) \sum_{i=1}^{n}\left(\prod_{l=2}^{k} \mathcal{D}_{j}\left(\alpha_{l}\right)\right) . \\
& \quad \cdot F_{\mathcal{Y}_{1}, \ldots, \mathcal{Y}_{n}}\left(w_{1}, \ldots, w_{i-1}, L(0)_{n} w_{i}, w_{i+1}, \ldots, w_{n} ; z_{1}, \ldots, z_{n} ; q\right) .
\end{aligned}
$$

Remark 2.9. Using this notation, (2.22) can be written as

$$
\psi_{\mathcal{Y}_{1}, \ldots, \mathcal{Y}_{n}}\left(\mathcal{Q}_{j}\left(1 \otimes w_{1} \otimes \ldots \otimes w_{n}\right)\right)=\mathcal{D}_{j}\left(\sum_{i=1}^{n} \mathrm{wt} w_{i}\right) \psi_{\mathcal{Y}_{1}, \ldots, \mathcal{Y}_{n}}\left(1 \otimes w_{1} \otimes \ldots \otimes w_{n}\right)
$$

We now want to extend $Q_{j}$ to a map $\mathcal{Q}_{j}: T \rightarrow T$ such that, for any $t \in T$ of modular weight $\alpha$,

$$
\psi_{\mathcal{Y}_{1}, \ldots, \mathcal{Y}_{n}}\left(\mathcal{Q}_{j}(t)\right)=\mathcal{D}_{j}(\alpha) \psi_{\mathcal{Y}_{1}, \ldots, \mathcal{Y}_{n}}(t)
$$

This is necessary since we will need to apply $\psi_{\mathcal{Y}_{1}, \ldots, \mathcal{Y}_{n}}$ to repeated iterations of the map $\mathcal{Q}_{j}$ on the element $1 \otimes w_{1} \otimes \ldots \otimes w_{n}$ for homogeneous elements $w_{1}, \ldots, w_{n}$; and while the elements of $T$ obtained this way are not homogeneous in the conformal grading, they have a well defined modular weight. 
Definition 2.10. We define functions $\vartheta_{j}: R \rightarrow R$ for $j=1, \ldots, n$ in the following way: let

$$
\vartheta_{j}\left(\tilde{G}_{k}(q)\right)=(2 \pi i)^{2} q \frac{\partial}{\partial q} \tilde{G}_{k}(q)+k \tilde{G}_{2}(q) \tilde{G}_{k}(q)
$$

and for the formal series $\tilde{\wp}_{m}\left(z_{r}-z_{s} ; q_{\tau}\right)$ with $1 \leq r \neq s \leq n$ and any $m \geq 2$, and $j=1, \ldots, n$, we define $\vartheta_{j}\left(\tilde{\wp}_{m}\right)$ by

$$
\begin{aligned}
\vartheta_{j}\left(\tilde{\wp}_{m}\left(z_{r}-z_{s} ; q\right)\right) \\
=(2 \pi i)^{2} q \frac{\partial}{\partial q} \tilde{\wp}_{m}\left(z_{r}-z_{s} ; q\right)+m \tilde{G}_{2}(q) \tilde{\wp}_{m}\left(z_{r}-z_{s} ; q\right) \\
\quad-m \tilde{G}_{2}(q)\left(z_{r}-z_{s}\right) \tilde{\wp}_{m+1}\left(z_{r}-z_{s} ; q\right) \\
\quad+m \tilde{\rho}_{m+1}\left(z_{r}-z_{s} ; q\right)\left(\tilde{\wp}_{1}\left(z_{r}-z_{j} ; q\right)-\tilde{\wp}_{1}\left(z_{s}-z_{j} ; q\right)\right)
\end{aligned}
$$

if $j \notin\{r, s\}$; and by

$$
\begin{aligned}
\vartheta_{j}\left(\tilde{\wp}_{m}\left(z_{j}-z_{s} ; q\right)\right) \\
=(2 \pi i)^{2} q \frac{\partial}{\partial q} \tilde{\wp}_{m}\left(z_{j}-z_{s} ; q\right)+m \tilde{G}_{2}(q) \tilde{\wp}_{m}\left(z_{j}-z_{s} ; q\right) \\
\quad-m \tilde{G}_{2}(q)\left(z_{j}-z_{s}\right) \tilde{\wp}_{m+1}\left(z_{j}-z_{s} ; q\right) \\
\quad-m \tilde{\wp}_{m+1}\left(z_{j}-z_{s} ; q\right) \tilde{\wp}_{1}\left(z_{s}-z_{j} ; q\right)
\end{aligned}
$$

if $j=r$, and we extend $\vartheta_{j}$ as a derivation on the ring $R$.

Proposition 2.11. Let $\varphi\left(z_{1}, \ldots, z_{n} ; q\right)=\vartheta_{j}\left(\tilde{\wp}_{m}\left(z_{r}-z_{s} ; q\right)\right)$; then $\varphi\left(z_{1}, \ldots, z_{n} ; q_{\tau}\right)$ converges uniformly to an elliptic function in the variables $z_{1}, \ldots, z_{n}$ with possible poles at $z_{r}=z_{s}+$ $m \tau+n, n, m \in \mathbb{Z}, r, s=1, \ldots, n$. Moreover, for any $g \in S L_{2}(\mathbb{Z})$, if

$$
g=\left(\begin{array}{ll}
\alpha & \beta \\
\gamma & \delta
\end{array}\right)
$$

we have

$$
\varphi\left(\frac{z_{1}}{\gamma \tau+\delta}, \ldots, \frac{z_{n}}{\gamma \tau+\delta} ; \frac{\alpha \tau+\beta}{\gamma \tau+\delta}\right)=(\gamma \tau+\delta)^{m+2} \varphi\left(z_{1}, \ldots, z_{n} ; \tau\right)
$$

Proof. Easy computation using transformation properties of $\wp_{m}$ and $G_{k}$ under the action of $S L_{2}(\mathbb{Z})$.

In particular, $\vartheta_{j}$ is indeed a map from $R$ to $R$ and if $f \in R$ has modular weight $p$, then $\vartheta_{j}(f)$ has modular weight $p+2$. We then consider the $\mathbb{C}$-linear maps $\mathcal{Q}_{j}: T \rightarrow T$ for $j=1, \ldots, n$ defined by

$$
\mathcal{Q}_{j}\left(f(q) \otimes w_{1} \otimes \ldots \otimes w_{n}\right)
$$




$$
=f(q) \cdot Q_{j}\left(1 \otimes w_{1} \otimes \ldots \otimes w_{n}\right)+\vartheta_{j}(f(q)) \otimes w_{1} \otimes \ldots \otimes w_{n}
$$

note that if the modular weight of $t \in T$ is $\alpha$, then the modular weight of $\mathcal{Q}_{j}(t)$ is $\alpha+2$.

Proposition 2.12. Let $t \in T$ be an element of modular weight $\alpha$. Then

$$
\psi_{\mathcal{Y}_{1}, \ldots, \mathcal{Y}_{n}}\left(\mathcal{Q}_{j}(t)\right)=\mathcal{D}_{j}(\alpha) \cdot \psi_{\mathcal{Y}_{1}, \ldots, \mathcal{Y}_{n}}(t)
$$

Proof. It is enough to prove this for elements $t$ of the form $\tilde{G}_{k}(q) \otimes w_{1} \otimes \ldots \otimes w_{n}$ or $\tilde{\wp}_{m}\left(z_{i}-\right.$ $\left.z_{j} ; q\right) \otimes w_{1} \otimes \ldots \otimes w_{n}$. Suppose $t$ is of the first form with $\sum_{i=1}^{n}$ wt $w_{i}=s$ with $k+s=\alpha$. Then

$$
\begin{aligned}
\psi_{\mathcal{Y}_{1}, \ldots, \mathcal{Y}_{n}}\left(\mathcal{Q}_{j}(t)\right) & \\
= & \psi_{\mathcal{Y}_{1}, \ldots, \mathcal{Y}_{n}}\left(\tilde{G}_{k}(q) Q_{j}\left(1 \otimes w_{1} \otimes \ldots \otimes w_{n}\right)+\theta\left(\tilde{G}_{k}(q)\right) \otimes w_{1} \otimes \ldots \otimes w_{n}\right) \\
= & \tilde{G}_{k}(q) \mathcal{D}_{j}(s) F_{\mathcal{Y}_{1}, \ldots, \mathcal{Y}_{n}}\left(w_{1}, \ldots, w_{n} ; z_{1}, \ldots, z_{n} ; q\right) \\
& +\left((2 \pi i)^{2} q \frac{\partial}{\partial q} \tilde{G}_{k}(q)+k \tilde{G}_{2}(q) \tilde{G}_{k}(q)\right) F_{\mathcal{Y}_{1}, \ldots, \mathcal{Y}_{n}}\left(w_{1}, \ldots, w_{n} ; z_{1}, \ldots, z_{n} ; q\right) \\
= & \mathcal{D}_{j}(s+k) \tilde{G}_{k}(q) F_{\mathcal{Y}_{1}, \ldots, \mathcal{Y}_{n}}\left(w_{1}, \ldots, w_{n} ; z_{1}, \ldots, z_{n} ; q\right) \\
= & \mathcal{D}_{j}(\alpha) \psi_{\mathcal{Y}_{1}, \ldots, \mathcal{Y}_{n}}(t) .
\end{aligned}
$$

The proof of the other case is a similar computation.

Proposition 2.13. Let $\alpha=\sum_{i=1}^{n}$ wt $w_{i}$; then for any $s \in \mathbb{N}$, we have

$$
\begin{aligned}
& \psi_{\mathcal{Y}_{1}, \ldots, \mathcal{Y}_{n}}\left(Q_{j}^{s}\left(1 \otimes w_{1} \otimes \ldots \otimes w_{n}\right)\right) \\
& \quad=\prod_{i=1}^{s} \mathcal{D}_{j}(\alpha+2(s-i)) \cdot F_{\mathcal{Y}_{1}, \ldots, \mathcal{Y}_{n}}\left(w_{1}, \ldots, w_{n} ; z_{1}, \ldots, z_{n} ; q\right) .
\end{aligned}
$$

Proof. We proceed by induction on $s$; the base case $s=0$ follows by definition of $\psi_{\mathcal{Y}_{1}, \ldots, \mathcal{Y}_{n}}$. Now suppose the claim holds for $s-1$; then by (2.19), and since $Q^{k}\left(1 \otimes w_{1} \otimes \ldots \otimes w_{n}\right)$ has modular weight equal to $\alpha+2(k-1)$,

$$
\begin{aligned}
& \psi_{\mathcal{Y}_{1}, \ldots, \mathcal{Y}_{n}}\left(Q_{j}^{s}\left(1 \otimes w_{1} \otimes \ldots \otimes w_{n}\right)\right)=\mathcal{O}_{j}(\alpha+2(s-1)) \psi_{\mathcal{Y}_{1}, \ldots, \mathcal{Y}_{n}}\left(Q_{j}^{s-1}\left(1 \otimes w_{1} \otimes \ldots \otimes w_{n}\right)\right) \\
& \quad+\tilde{G}_{2}(q) \sum_{i=1}^{n} \psi_{\mathcal{Y}_{1}, \ldots, \mathcal{Y}_{i-1}, \mathcal{Y}_{i}^{(1)}, \mathcal{Y}_{i+1}, \ldots, \mathcal{Y}_{n}}\left(Q_{j}^{s-1}\left(1 \otimes w_{1} \otimes \ldots \otimes w_{n}\right)\right) \\
&=\mathcal{O}_{j}(\alpha+2(s-1))\left(\prod_{l=1}^{s-1} \mathcal{D}_{j}(\alpha+2(s-1-l))\right) \\
& \quad F_{\mathcal{Y}_{1}, \ldots, \mathcal{Y}_{n}}\left(w_{1}, \ldots, w_{n} ; z_{1}, \ldots, z_{n} ; q\right) \\
&+\tilde{G}_{2}(q) \sum_{i=1}^{n}\left(\prod_{l=1}^{s-1} \mathcal{D}_{j}(\alpha+2(s-1-l))\right)
\end{aligned}
$$




$$
\begin{aligned}
& \quad \cdot F_{\mathcal{Y}_{1}, \ldots, \mathcal{Y}_{n}}\left(w_{1}, \ldots, w_{i-1}, L(0)_{n} w_{i}, w_{i+1}, \ldots, w_{n} ; z_{1}, \ldots, z_{n} ; q\right) \\
& =\mathcal{O}_{j}(\alpha+2(s-1))\left(\prod_{l=2}^{s} \mathcal{D}_{j}(\alpha+2(s-l))\right) \\
& \quad \cdot F_{\mathcal{Y}_{1}, \ldots, \mathcal{Y}_{n}}\left(w_{1}, \ldots, w_{n} ; z_{1}, \ldots, z_{n} ; q\right) \\
& +\tilde{G}_{2}(q) \sum_{i=1}^{n}\left(\prod_{l=2}^{s} \mathcal{D}_{j}(\alpha+2(s-l))\right) \\
& \quad \cdot F_{\mathcal{Y}_{1}, \ldots, \mathcal{Y}_{n}}\left(w_{1}, \ldots, w_{i-1}, L(0)_{n} w_{i}, w_{i+1}, \ldots, w_{n} ; z_{1}, \ldots, z_{n} ; q\right) \\
& =\prod_{l=1}^{s} \mathcal{D}_{j}(\alpha+2(s-l)) \cdot F_{\mathcal{Y}_{1}, \ldots, \mathcal{Y}_{n}}\left(w_{1}, \ldots, w_{n} ; z_{1}, \ldots, z_{n} ; q\right),
\end{aligned}
$$

which concludes the proof.

Proposition 2.14. Let $W_{1}, \ldots W_{n}$ be grading-restricted generalized modules for the vertex operator algebra $V$, and consider homogeneous $w_{i} \in W_{i}$ for $i=1, \ldots n$; let $\alpha=\sum_{i=1}^{n}$ wt $w_{i}$. Then there exist elements $b_{p, i} \in R_{2 p}$ for $p=1, \ldots, m$ such that for any $V$-P-bimodules $\tilde{W}_{j}$ and P-intertwining operators $\mathcal{Y}_{j}$ of type $\left(\begin{array}{c}\tilde{W}_{j-1} \\ W_{j} \tilde{W}_{j}\end{array}\right), j=1, \ldots, n$, with $\tilde{W}_{0}=\tilde{W}_{n}$ projective as a right $P$-module, the series

$$
\varphi=F_{\mathcal{Y}_{1}, \ldots, \mathcal{Y}_{n}}\left(w_{1}, \ldots, w_{n} ; z_{1}, \ldots z_{n} ; q\right)
$$

satisfies the differential equations

$$
\begin{aligned}
& \prod_{l=1}^{m} \mathcal{D}_{j}(\alpha+2(m-l)) \varphi \\
& \quad+\sum_{p=1}^{m} b_{p, j}\left(z_{1}, \ldots, z_{n} ; q\right) \prod_{l=1}^{m-p} \mathcal{D}_{j}(\alpha+2(m-p-l)) \varphi=0
\end{aligned}
$$

for $j=1, \ldots, n$, in the region $1>\left|q_{z_{1}}\right|>\ldots>\left|q_{z_{1}}\right|>|q|>0$.

Moreover, for any $k=1, \ldots, n$, the series

$$
\begin{aligned}
& \varphi_{k}\left(z_{1}, \ldots, z_{n} ; q\right) \\
& \quad=F_{\mathcal{Y}_{1}, \ldots, \mathcal{Y}_{n}}^{\phi}\left(w_{1}, \ldots, w_{k-1}, L(0)_{n} w_{k}, w_{k+1}, \ldots, w_{n} ; z_{1}, \ldots z_{n} ; q\right)
\end{aligned}
$$

also satisfies (2.25), for the same choice of elements $b_{p, i}$.

Proof. Consider the $R$-submodule $M$ of $T / J$ generated by the elements

$$
\mathcal{Q}_{j}^{k}\left(1 \otimes w_{1} \otimes \ldots \otimes w_{n}\right)+J
$$

for $k \in \mathbb{N}$. Since $T / J$ is finitely generated and $R$ is noetherian, $M$ is also finitely generated; therefore, there exists $m$ and elements $b_{p, j}\left(z_{1}, \ldots, z_{n} ; q\right) \in R, p=1, \ldots, m$ such that

$$
\mathcal{Q}_{j}^{m}\left(1 \otimes w_{1} \otimes \ldots \otimes w_{n}\right)
$$




$$
+\sum_{p=1}^{m} b_{p, j}\left(z_{1}, \ldots, z_{n} ; q\right) \mathcal{Q}_{j}^{m-p}\left(1 \otimes w_{1} \otimes \ldots \otimes w_{n}\right) \in J
$$

Since the modular weight of $\mathcal{Q}_{j}^{i}\left(1 \otimes w_{1} \otimes \ldots \otimes w_{n}\right)$ is $\alpha+2 i$, we can choose $b_{p, j}$ in $R_{2 p}$; then applying $\psi_{\mathcal{Y}_{1}, \ldots, \mathcal{Y}_{n}}$ to both sides of the equation and applying (2.24), we obtain (2.25).

The last part of the proposition follows by applying the first part to the intertwining operators $\mathcal{Y}_{1}, \ldots, \mathcal{Y}_{k-1}, \mathcal{Y}_{k}^{(1)}, \mathcal{Y}_{k+1}, \ldots, Y_{n}$.

Remark 2.15. Note that the coefficients $b_{p, j}$ only depend on the elements $w_{1}, \ldots, w_{n}$; in particular, they do not depend on the choice of $P$ and $\phi$ in the definition of the pseudotrace.

Remark 2.16. For $i_{j} \in \mathbb{N}$, and $i=1, \ldots, n$, let

$$
\begin{aligned}
& \varphi_{i_{1}, \ldots, i_{n}}\left(z_{1}, \ldots, z_{n} ; q\right) \\
& \quad=F_{\mathcal{Y}_{1}, \ldots, \mathcal{Y}_{n}}\left(L(0)_{n}^{i_{1}} w_{1}, \ldots, L(0)_{n}^{i_{n}} w_{n} ; z_{1}, \ldots, z_{n} ; q\right),
\end{aligned}
$$

The differential equation (2.25) depends on all the functions $\varphi_{i_{1}, \ldots, i_{n}}$; therefore, we obtain a system of differential equations of which $\left\{\varphi_{i_{1}, \ldots, i_{n}} \mid i_{j} \in \mathbb{N}, j=1, \ldots, n\right\}$ is a solution. Since $L(0)_{n}$ is locally nilpotent, this system of equations is finite; if the modules $W_{1}, \ldots, W_{n}$ all have length smaller than $l$, then we obtain a system for the $l(n+1)$ functions $\left\{\varphi_{i_{1}, \ldots, i_{n}} \mid i_{j}=\right.$ $0, \ldots, l, j=1, \ldots, n\}$. If the modules are ordinary modules the system decouples and we obtain the equations in $[\mathrm{H} 2$.

Proposition 2.17. The series

$$
F_{\mathcal{Y}_{1}, \ldots, \mathcal{Y}_{n}}\left(w_{1}, \ldots, w_{n} ; z_{1}, \ldots, z_{n} ; q_{\tau}\right)
$$

is absolutely convergent in the region $1>\left|q_{z_{1}}\right|>\ldots>\left|q_{z_{n}}\right|>\left|q_{\tau}\right|>0 \mid$ and can be extended to a multivalued analytic function in the region $\Im(\tau)>0, z_{i} \neq z_{j}+l+m \tau$ for $i \neq j, l, m \in \mathbb{Z}$.

Proof. For fixed $z_{1}, \ldots, z_{n}$ such that $\left|q_{z_{1}}\right|>\ldots>\left|q_{z_{n}}\right|>0$, the coefficients in the variable $q$, $\log q$ of the series

$$
F_{\mathcal{Y}_{1}, \ldots, \mathcal{Y}_{n}}\left(w_{1}, \ldots, w_{n} ; z_{1}, \ldots, z_{n} ; q\right)
$$

are absolutely convergent, and the series satisfies a system of differential equations with a regular singular point at $q=0$ and analytic coefficients. Since the coefficients of the differential equations are analytic functions in $z_{1}, \ldots, z_{n}, q_{\tau}$, with possible singularities in the region $\Im(\tau)>0, z_{i} \neq z_{j}+l+m \tau$ for $i \neq j$ and $l, m \in \mathbb{Z}$, the solutions of the system (2.25) can be extended to an analytic (multivalued) function in the same region.

We will call genus-one correlation functions the analytic extensions of (2.26) to the region $\Im(\tau)>0, z_{i} \neq z_{j}+l+m \tau$ for $i \neq j, l, m \in \mathbb{Z}$, and we will denote them by

$$
\bar{F}_{\mathcal{Y}_{1}, \ldots, \mathcal{Y}_{n}}^{\phi}\left(w_{1}, \ldots, w_{n} ; z_{1}, \ldots, z_{n} ; q_{\tau}\right)
$$


Proposition 2.18 (Genus-one commutativity). Let $W_{i}$ be grading-restricted generalized $V$ modules, $\tilde{W}_{i}$ be $V$-P bimodules and $\mathcal{Y}_{i}$ logarithmic intertwining operators of type $\left(\begin{array}{c}\tilde{W}_{i-1} \\ W_{i} \tilde{W}_{i-1}\end{array}\right)$ for $i=1, \ldots, n$, with $\tilde{W}_{0}=\tilde{W}_{n}$ projective as a right $P$-module. Then for any $k \leq n-1$, there exists a $V$-P-bimodules $\hat{W}_{k}$, and logarithmic intertwining operators $\hat{\mathcal{Y}}_{k}, \hat{\mathcal{Y}}_{k+1}$ of type $\left(\begin{array}{c}\hat{W}_{k} \\ W_{k} \tilde{W}_{k+1}\end{array}\right),\left(\begin{array}{c}\tilde{W}_{k-1} \\ W_{k+1} \hat{W}_{k}\end{array}\right)$ such that

$$
\begin{array}{r}
\bar{F}_{\mathcal{Y}_{1}, \ldots, \mathcal{Y}_{n}}^{\phi}\left(w_{1}, \ldots, w_{n} ; z_{1}, \ldots, z_{n} ; \tau\right) \\
=\bar{F}_{\mathcal{Y}_{1}, \ldots, \mathcal{Y}_{k-1}, \hat{\mathcal{Y}}_{k+1}, \hat{Y}_{k}, \mathcal{Y}_{k+2}, \ldots \mathcal{Y}_{n}}\left(\begin{array}{r}
w_{1}, \ldots, w_{k-1}, w_{k+1}, w_{k}, w_{k+2} \ldots, w_{n} ; \\
\left.z_{1}, \ldots, z_{i-1}, z_{i+1}, z_{i}, z_{i+2}, \ldots, z_{n} ; \tau\right)
\end{array}\right.
\end{array}
$$

as multivalued analytic functions.

Proof. Follows from commutativity for $P$ intertwining operators.

Proposition 2.19 (Genus-one associativity). Let $W_{i}$ be grading-restricted generalized $V$ modules, $\tilde{W}_{i}$ be $V$-P-bimodules and $\mathcal{Y}_{i}$ logarithmic intertwining operators of type $\left(\begin{array}{c}\tilde{W}_{i-1} \\ W_{i} \tilde{W}_{i-1}\end{array}\right)$ for $i=1, \ldots, n$, with $\tilde{W}_{0}=\tilde{W}_{n}$ projective as a right $P$-module. Then for any $k \leq n-1$, there exists a $V$-P-bimodules $\hat{W}_{k}$, and logarithmic intertwining operators $\hat{\mathcal{Y}}_{k}, \hat{\mathcal{Y}}_{k+1}$ of type $\left(\begin{array}{c}\hat{W}_{k} \\ W_{k} W_{k+1}\end{array}\right),\left(\begin{array}{c}\tilde{W}_{k-1} \\ \hat{W}_{k} \tilde{W}_{k+1}\end{array}\right)$ such that the series

$$
\begin{gathered}
\bar{F}_{\mathcal{Y}_{1}, \ldots, \mathcal{Y}_{k-1}, \hat{\mathcal{Y}}_{k+1}, \mathcal{Y}_{k+2}, \ldots, \mathcal{Y}_{n}}\left(w_{1}, \ldots, w_{k-1}, \hat{\mathcal{Y}}\left(w_{k}, z_{k}-z_{k+1}\right) w_{k+1},\right. \\
\left.w_{k+2}, \ldots, w_{n} ; z_{1}, \ldots, z_{n} ; \tau\right) \\
=\sum_{r \in \mathbb{R}} \bar{F}_{\mathcal{Y}_{1}, \ldots, \mathcal{Y}_{k-1}, \hat{\mathcal{Y}}_{k+1}, \mathcal{Y}_{k+2}, \ldots, \mathcal{Y}_{n}}\left(w_{1}, \ldots, w_{k-1}, P_{r}\left(\hat{\mathcal{Y}}\left(w_{k}, z_{k}-z_{k+1}\right) w_{k+1}\right),\right. \\
\left.w_{k+2}, \ldots, w_{n} ; z_{1}, \ldots, z_{n} ; \tau\right)
\end{gathered}
$$

is absolutely convergent in the region

$$
1>\left|q_{z_{1}}\right|>\ldots\left|q_{z_{k-1}}\right|>\left|q_{z_{k+1}}\right| \ldots>\left|q_{z_{n}}\right|>\left|q_{\tau}\right|>0
$$

and $1>\left|q_{\left(z_{k}-z_{k+1}\right)}\right|>0$ and converges to $\bar{F}_{\mathcal{Y}_{1}, \ldots, \mathcal{Y}_{n}}^{\phi}\left(w_{1}, \ldots, w_{n} ; z_{1}, \ldots, z_{n} ; \tau\right)$ when $1>\left|q_{z_{1}}\right|>$ $\ldots>\left|q_{z_{n}}\right|>\left|q_{\tau}\right|>0$ and $\left|q_{\left(z_{k}-z_{k+1}\right)}\right|>1>\left|q_{\left(z_{k}-z_{k+1}\right)}-1\right|>0$.

Proof. By associativity for $P$-intertwining operators, there exist a $V$-P-bimodule $\tilde{W}_{k}$ and logarithmic intertwining operators $\hat{\mathcal{Y}}_{k}, \hat{\mathcal{Y}}_{k+1}$ of type $\left(\begin{array}{c}\hat{W}_{k} \\ W_{k} W_{k+1}\end{array}\right),\left(\begin{array}{c}\tilde{W}_{k-1} \\ \hat{W}_{k} \tilde{W}_{k+1}\end{array}\right)$ such that for any $z_{1}, \ldots, z_{n} \in \mathbb{C}$ satisfying $1>\left|q_{z_{1}}\right|>\ldots>\left|q_{z_{n}}\right|>0$ and $\left|q_{z_{k+1}}\right|>\left|q_{z_{k}}-q_{z_{k+1}}\right|>0$, and for any element $\tilde{w}_{n}^{\prime} \in \tilde{W}_{n}^{\prime}, \tilde{w}_{n} \in \tilde{W}_{n}$

$$
\begin{aligned}
&\left\langle w_{n}^{\prime}, \mathcal{Y}_{1}(\right.\left.\mathcal{U}\left(q_{z_{1}}\right) w_{1}, q_{z_{1}}\right) \ldots \mathcal{Y}_{k-1}\left(\mathcal{U}\left(q_{z_{k-1}}\right) w_{k-1}, q_{z_{k-1}}\right) . \\
& \cdot \hat{\mathcal{Y}}_{k+1}\left(\mathcal{U}\left(q_{z_{k+1}}\right) \hat{\mathcal{Y}}_{k}\left(w_{k}, z_{k}-z_{k+1}\right) w_{k+1}, q_{z_{k+1}}\right) . \\
& \cdot \mathcal{Y}_{k+2}\left(\mathcal{U}\left(q_{z_{k+2}}\right) w_{k+2}, q_{z_{k+2}}\right) \ldots \mathcal{Y}_{n}\left(\mathcal{U}\left(q_{z_{n}}\right) w_{n}, q_{z_{n}}\right) \tilde{w}_{n} \\
&=\left\langle\tilde{w}_{n}^{\prime}, \mathcal{Y}_{1}\left(\mathcal{U}\left(q_{z_{1}}\right) w_{1}, q_{z_{1}}\right) \ldots \mathcal{Y}_{n}\left(\mathcal{U}\left(q_{z_{n}}\right) w_{n}, q_{z_{n}}\right) \tilde{w}_{n}\right\rangle
\end{aligned}
$$


and therefore as series in $q$ and $\log q$,

$$
\begin{aligned}
\operatorname{tr}_{\tilde{W}_{n}}^{\phi} \mathcal{Y}_{1}\left(\mathcal{U}\left(q_{z_{1}}\right) w_{1}, q_{z_{1}}\right) \ldots \mathcal{Y}_{k-1}\left(\mathcal{U}\left(q_{z_{k-1}}\right) w_{k-1}, q_{z_{k-1}}\right) \cdot \\
\cdot \hat{\mathcal{Y}}_{k+1}\left(\mathcal{U}\left(q_{z_{k+1}}\right) \hat{\mathcal{Y}}_{k}\left(w_{k}, z_{k}-z_{k+1}\right) w_{k+1}, q_{z_{k+1}}\right) \cdot \\
\cdot \mathcal{Y}_{k+2}\left(\mathcal{U}\left(q_{z_{k+2}}\right) w_{k+2}, q_{z_{k+2}}\right) \ldots \mathcal{Y}_{n}\left(\mathcal{U}\left(q_{z_{n}}\right) w_{n}, q_{z_{n}}\right) q^{L(0)-\frac{c}{24}} \\
=\operatorname{tr}_{\tilde{W}_{n}}^{\phi} \mathcal{Y}_{1}\left(\mathcal{U}\left(q_{z_{1}}\right) w_{1}, q_{z_{1}}\right) \ldots \mathcal{Y}_{n}\left(\mathcal{U}\left(q_{z_{n}}\right) w_{n}, q_{z_{n}}\right) q^{L(0)-\frac{c}{24}}
\end{aligned}
$$

The right hand side is absolutely convergent when $q=q_{\tau}$ and $1>\left|q_{z_{1}}\right|>\ldots>\left|q_{z_{1}}\right|>\left|q_{\tau}\right|>$ 0 , the right hand side is also absolutely convergent if $\left|q_{z_{k}}\right|>\left|q_{z_{k}}-q_{z_{k+1}}\right|>0$, and satisfies the same system of differential equations as

$$
\operatorname{tr}_{\tilde{W}_{n}}^{\phi} \mathcal{Y}_{1}\left(\mathcal{U}\left(q_{z_{1}}\right) w_{1}, q_{z_{1}}\right) \ldots \mathcal{Y}_{n}\left(\mathcal{U}\left(q_{z_{n}}\right) w_{n}, q_{z_{n}}\right) q^{L(0)-\frac{c}{24}}
$$

So in this region the left hand side converges absolutely to a function that can be extended to the multivalued analytic function

$$
\bar{F}_{\mathcal{Y}_{1}, \ldots, \mathcal{Y}_{n}}^{\phi}\left(w_{1}, \ldots, w_{n} ; z_{1}, \ldots, z_{n}, \tau\right)
$$

which concludes the proof.

\subsection{Modular invariance of the space of solutions}

In this section we consider a space of functions which contains the solutions of the system of differential equations (2.25), and we define an action of the group $S L_{2}(\mathbb{Z})$ on the elements of this space. We prove that the space of solutions of (2.25) is invariant under this action.

We will introduce the following notations: let $\chi$ be the space of sequences (indexed by $n$ indices) of analytic multivalued functions in the variables $z_{1}, \ldots, z_{n}, \tau$, on the region $\Im(\tau)>0, z_{i} \neq z_{j}+n \tau+m$ for $n, m \in \mathbb{N}, i \neq j$

$$
\Phi=\Phi\left(z_{1}, \ldots, z_{n} ; \tau\right)=\left(\phi_{i_{1}, \ldots, i_{n}}\left(z_{1}, \ldots, z_{n} ; \tau\right)\right)_{i_{1}, \ldots, i_{n} \in \mathbb{N}}
$$

with preferred branches on the region $1>\left|q_{z_{1}}\right|>\ldots>\left|q_{z_{n}}\right|>\left|q_{\tau}\right|>0$, such that $\phi_{i_{1}, \ldots, i_{n}} \equiv 0$ whenever $\max \left\{i_{1}, \ldots, i_{n}\right\}$ is sufficiently large. We will also denote an element $\Phi$ of $\chi$ by $\left(\phi_{i_{1}, \ldots, i_{n}}\right)$ or using a multi-index notation $\left(\phi_{\mu}\right)$ for $\mu$ ranging over $\mathbb{N}^{n}$. The sum of sequences of this kind is defined component by component, so that if $\Phi^{1}, \Phi^{2}$ are two elements of $\chi$, the $\mu$-th component of $\Phi^{1}+\Phi^{2}$ is $\left(\phi_{\mu}^{1}+\phi_{\mu}^{2}\right)$ and similarly we can define the product by another analytic function. Moreover, we extend differential operators to $\chi$ component-wise:

$$
\left(\frac{\partial}{\partial z_{i}} \Phi\right)_{\mu}=\frac{\partial \phi_{\mu}}{\partial z_{i}}, i=1, \ldots, n .
$$

For $j=1, \ldots, n$, let $d_{j}: \chi \rightarrow \chi$ be the shift operator on the $j$-th coordinate defined by

$$
\left(d_{j} \Phi\right)_{i_{1}, \ldots, i_{n}}=\phi_{i_{1}, \ldots, i_{j-1}, i_{j}+1, i_{j+1}, \ldots i_{n}} .
$$


Note that for any $\Phi \in \chi, d_{j}^{k} \Phi=0$ if $k$ is large enough; therefore for any function $f\left(z_{1}, \ldots, z_{n} ; \tau\right)$, the operator

$$
e^{f\left(z_{1}, \ldots, z_{n} ; \tau\right) d_{j}}=\sum_{k=0}^{\infty} \frac{f^{k}\left(z_{1}, \ldots, z_{n} ; \tau\right)}{k !} d_{j}^{k}
$$

is well defined.

Now, for $\alpha \in \mathbb{C}$ and $j=1, \ldots n$, we define $\mathcal{D}_{j}(\alpha): \chi \rightarrow \chi$ by

$$
\mathcal{D}_{j}(\alpha)=\mathcal{O}_{j}(\alpha)+G_{2}(\tau) \sum_{i=1}^{n} d_{i}
$$

where $\mathcal{O}_{j}(\alpha)$ is defined as in (2.23) $\left(\right.$ with $\left.(2 \pi i)^{2} q \frac{\partial}{\partial q}=2 \pi i \frac{\partial}{\partial \tau}\right)$ and extended component-wise to $\chi$.

Remark 2.20. Using the notation as in remark (2.16), for fixed elements $w_{i} \in W_{i}, i=$ $1, \ldots, n$, and logarithmic intertwining operators $\mathcal{Y}_{1}, \ldots \mathcal{Y}_{n}$, one can consider the element of $\chi$ :

$$
\Phi=\left(\varphi_{i_{1}, \ldots, i_{n}}\right)_{i_{1}, \ldots, i_{n} \in \mathbb{N}}
$$

Then if $b_{p, j}\left(z_{1}, \ldots, z_{n} ; \tau\right) j=1, \ldots, n$ are defined as in Proposition 2.14, by the same proposition we have

$$
\begin{aligned}
& \left(\prod_{l=1}^{m} \mathcal{D}_{j}(\alpha+2(m-l))\right. \\
& \left.\quad+\sum_{p=1}^{m} b_{p, j}\left(z_{1}, \ldots, z_{n} ; q\right) \prod_{l=1}^{m-p} \mathcal{D}_{j}(\alpha+2(m-p-l))\right) \Phi=0 .
\end{aligned}
$$

Definition 2.21 $\left(S L_{2}(\mathbb{Z})\right.$ action). Let $a \in \mathbb{C}$, and consider an element $g$ in $S L_{2}(\mathbb{Z})$,

$$
g=\left(\begin{array}{ll}
\alpha & \beta \\
\gamma & \delta
\end{array}\right)
$$

For $\Phi \in \chi$, we define

$$
\begin{aligned}
& \left.\Phi\right|_{g, a}\left(z_{1}, \ldots, z_{n} ; \tau\right) \\
& \quad=\left(\frac{1}{\gamma \tau+\delta}\right)^{a} \prod_{i=1}^{n} e^{-\log (\gamma \tau+\delta) d_{i}} \Phi\left(\frac{z_{1}}{\gamma \tau+\delta}, \ldots, \frac{z_{n}}{\gamma \tau+\delta} ; \frac{\alpha \tau+\beta}{\gamma \tau+\delta}\right)
\end{aligned}
$$

Proposition 2.22. For any $a \in \mathbb{C}$, (2.28) defines an action of the group $S L_{2}(\mathbb{Z})$ on the space $\chi$. 
We will also use the notation $z^{\prime}, \tau^{\prime}$ to denote $\frac{z}{\gamma \tau+\delta}$ and $\frac{\alpha \tau+\beta}{\gamma \tau+\delta}$ respectively. Note that for any function $f\left(z_{1}, \ldots, z_{n} ; q\right), g \in S L_{2}(\mathbb{Z})$, and any $\Phi \in \chi$,

$$
\left.(f \Phi)\right|_{g, a}=\left.f\left(z_{1}^{\prime}, \ldots, z_{n}^{\prime}, \tau^{\prime}\right) \Phi\right|_{g, a} ;
$$

in particular, if $f \in R_{p}$, then $f\left(z_{1}^{\prime}, \ldots, z_{n}^{\prime} ; \tau^{\prime}\right)=(\gamma \tau+\delta)^{p} f\left(z_{1}, \ldots, z_{n} ; \tau\right)$ and thus

$$
\left.(f \Phi)\right|_{g, a}=\left.f\left(z_{1}, \ldots, z_{n}, \tau\right) \Phi\right|_{g, a-p} .
$$

Proposition 2.23. Let $\Phi \in \chi, a \in \mathbb{C}$ and $g \in G$. Then

$$
\mathcal{D}_{j}(a)\left(\left.\Phi\right|_{g, a}\right)=\left.\left(\mathcal{D}_{j}(a) \Phi\right)\right|_{g, a+2}
$$

Proof. This is just a straightforward computation, using the transformation properties of the functions $G_{2}(\tau)$ and $\wp_{1}(z ; \tau)$ : for simplicity we use the notation

$$
\begin{aligned}
& e^{d}=\prod_{i=1}^{n} e^{-\log (\gamma \tau+\delta) d_{i}} \\
& \mathcal{D}_{j}(a)\left(\left.\Phi\right|_{g, a}\right)=\mathcal{D}_{j}(a)\left(\left(\frac{1}{\gamma \tau+\delta}\right)^{a} e^{d} \Phi\left(z_{1}^{\prime}, \ldots, z_{n}^{\prime} ; \tau^{\prime}\right)\right) \\
& =\left((2 \pi i) \frac{\partial}{\partial \tau}+G_{2}(\tau)\left(a+\sum_{i=1}^{n} d_{i}\right)+G_{2}(\tau) \sum_{i=1}^{n} z_{i} \frac{\partial}{\partial z_{i}}-\sum_{i \neq j} \wp_{1}\left(z_{i}-z_{j} ; \tau\right) \frac{\partial}{\partial z_{i}}\right) . \\
& \cdot\left(\left(\frac{1}{\gamma \tau+\delta}\right)^{a} \prod_{i=1}^{n} e^{-\log (\gamma \tau+\delta) d_{i}} \Phi\left(z_{1}^{\prime} \ldots, z_{n}^{\prime} ; \tau^{\prime}\right)\right) \\
& =-(2 \pi i) \gamma a\left(\frac{1}{\gamma \tau+\delta}\right)^{a+1} e^{d} \Phi\left(z_{1}^{\prime}, \ldots, z_{n}^{\prime}, \tau^{\prime}\right) \\
& -(2 \pi i) \gamma\left(\frac{1}{\gamma \tau+\delta}\right)^{a+1} e^{d} \sum_{i=1}^{n} d_{i} \Phi\left(z_{1}^{\prime}, \ldots, z_{n}^{\prime} \tau^{\prime}\right) \\
& -(2 \pi i) \gamma\left(\frac{1}{\gamma \tau+\delta}\right)^{a+1} e^{d} \sum_{i=1}^{n} z_{i}^{\prime} \frac{\partial \Phi}{\partial z_{i}}\left(z_{1}^{\prime}, \ldots, z_{n}^{\prime}, \tau^{\prime}\right) \\
& +(2 \pi i)\left(\frac{1}{\gamma \tau+\delta}\right)^{a+2} e^{d} \frac{\partial \Phi}{\partial \tau}\left(z_{1}^{\prime} \ldots, z_{n}^{\prime}, \tau^{\prime}\right) \\
& +\left(G_{2}\left(\tau^{\prime}\right)\left(\frac{1}{\gamma \tau+\delta}\right)^{2}+2 \pi i \gamma\left(\frac{1}{\gamma \tau+\delta}\right)\right)\left(a+\sum_{i=1}^{n} d_{i}\right) . \\
& \cdot\left(\frac{1}{\gamma \tau+\delta}\right)^{a} e^{d} \Phi\left(z_{1}^{\prime}, \ldots, z_{n}^{\prime} ; \tau^{\prime}\right) \\
& +\left(G_{2}\left(\tau^{\prime}\right)\left(\frac{1}{\gamma \tau+\delta}\right)^{2}+2 \pi i \gamma\left(\frac{1}{\gamma \tau+\delta}\right)\right) \text {. }
\end{aligned}
$$




$$
\begin{aligned}
& \cdot\left(\frac{1}{\gamma \tau+\delta}\right)^{a} e^{d} \sum_{i=1}^{n} z_{i}^{\prime} \frac{\partial \Phi}{\partial z_{i}}\left(z_{1}^{\prime}, \ldots, z_{n}^{\prime}, \tau^{\prime}\right) \\
& -\left(\frac{1}{\gamma \tau+\delta}\right)^{a+2} e^{d} \sum_{i \neq j} \wp_{1}\left(z_{i}^{\prime}-z_{j}^{\prime} ; \tau^{\prime}\right) \frac{\partial \Phi}{\partial z_{i}}\left(z_{1}^{\prime}, \ldots, z_{n}^{\prime}, \tau^{\prime}\right) \\
& =\left(\frac{1}{\gamma \tau+\delta}\right)^{a+2} e^{d} \cdot \\
& \quad\left((2 \pi i) \frac{\partial \Phi}{\partial \tau}\left(z_{1}^{\prime}, \ldots, z_{n}^{\prime}, \tau^{\prime}\right)+G_{2}\left(\tau^{\prime}\right)\left(a+\sum_{i=1}^{n} d_{i}\right) \Phi\left(z_{1}^{\prime}, \ldots, z_{n}^{\prime} ; \tau^{\prime}\right)\right. \\
& \left.\quad+G_{2}\left(\tau^{\prime}\right) \sum_{i=1}^{n} z_{i}^{\prime} \frac{\partial \Phi}{\partial z_{i}}\left(z_{1}^{\prime}, \ldots, z_{n}^{\prime}, \tau^{\prime}\right)-\sum_{i \neq j} \wp_{1}\left(z_{i}^{\prime}-z_{j}^{\prime} ; \tau^{\prime}\right) \frac{\partial \Phi}{\partial z_{i}}\left(z_{1}^{\prime}, \ldots, z_{n}^{\prime}, \tau^{\prime}\right)\right)
\end{aligned}
$$

which is equal to $\left.\left(\mathcal{D}_{j}(a) \Phi\right)\right|_{g, a+2}$, concluding the proof.

We can then prove the following

Proposition 2.24. Let $\Phi$ be a solution of the system of differential equations (2.27). Then for any $g \in S L_{2}(\mathbb{Z}),\left.\Phi\right|_{g, \alpha}$ is also a solution of the same system.

Proof. Just apply $\left.\right|_{g, \alpha+2 m}$ to both sides of (2.27); since $b_{p, j}$ belongs to $R_{2 p}$, we find

$$
\begin{aligned}
& \left.\left(b_{p, j}\left(z_{1}, \ldots, z_{n} ; q\right) \prod_{l=1}^{m-p} \mathcal{D}_{j}(\alpha+2(m-p-l)) \Phi\right)\right|_{g, \alpha+2 m} \\
& \quad=\left.b_{p, j}\left(z_{1}, \ldots, z_{n} ; q\right)\left(\prod_{l=1}^{m-p} \mathcal{D}_{j}(\alpha+2(m-p-l)) \Phi\right)\right|_{g, \alpha+2(m-p)}
\end{aligned}
$$

Now applying (2.29) several consecutive times, we obtain

$$
\begin{aligned}
& \left(\prod_{l=1}^{m} \mathcal{D}_{j}(\alpha+2(m-l))\right. \\
& \left.\quad+\sum_{p=1}^{m} b_{p, j}\left(z_{1}, \ldots, z_{n} ; q\right) \prod_{l=1}^{m-p} \mathcal{D}_{j}(\alpha+2(m-p-l))\right)\left.\Phi\right|_{g, \alpha}=0
\end{aligned}
$$

which concludes the proof.

\section{References}

[AF] F. Anderson and K. Fuller, Rings and Categories of Modules, Graduate Texts in Mathematics 13, Springer-Verlag, New York, Heidelberg, Berlin (1991). 
[AM1] D. Adamović and A. Milas, Logarithmic intertwining operators and $\mathcal{W}(2,2 p-1)$ algebras, Journal of Math. Physics 48, 073503 (2007).

[AM2] D. Adamović and A. Milas, On the triplet vertex algebra $\mathcal{W}(p)$, Adv. Math. 217, 2664-2699 (2008).

[AM3] D. Adamović and A. Milas, On $\mathcal{W}$-algebras associated to $(2, p)$ minimal models and their representations, IMRN 20, 3896-3934 (2010).

[AM4] D. Adamović and A. Milas, An analogue of modular BPZ equation in logarithmic (super)conformal field theory, Contemporary Mathematics 497, (2009) 1-17.

[Ar] Y. Arike, Some remarks on symmetric linear functions and pseudotrace maps, Proc. Japan Acad. Ser. A Math. Sci. 86 (2010), 119-124.

[AN] Y. Arike and K. Nagatomo, Some remarks on pseudo-trace functions for orbifold models associated with symplectic fermions. Int. J. Math. 24, (2013) 1350008

[B] R. E. Borcherds, Vertex algebras, Kac-Moody algebras, and the Monster, Proc. Natl. Acad. Sci. 83, 3068-3071 (1986).

[Br] M. Broué, Higman criterion revisited, Mich. J. Math. 58, (2009) 125-179.

[DLM1] Y. Dong, H. Li and G. Mason, Vertex operator algebras and associative algebras, J. Algebra 206, 67-96 (1998).

[DLM2] Y. Dong, H. Li and G. Mason, Modular invariance of trace functions in orbifold theory and generalized moonshine, Comm. Math. Phys. 214, 1-56 (2000).

[FH] F. Fiordalisi and Y.-Z. Huang, Modular invariance for logarithmic intertwining operators, in preparation.

[FHL] I. Frenkel, Y.-Z. Huang and J. Lepowsky, On axiomatic approaches to vertex operator algebras and modules, Memoirs Amer. Math. Soc., 104, 1993.

[FLM] I. Frenkel, J. Lepowsky and A. Meurman, Vertex Operator Algebras and the Monster, Pure and Appl. Math., Vol. 134, Academic Press, New York, 1988.

[H1] Y.-Z. Huang, Two-dimensional conformal geometry and vertex operator algebras, Progress in Mathematics, Vol. 148, Birkhäuser, Boston, 1997.

[H2] Y.-Z. Huang, Differential equations, duality and modular invariance, Comm. Contemp. Math. 7 (2005), 649-706.

[H3] Y.-Z. Huang, A theory of tensor product for module categories for a vertex operator algebra, IV, J. Pure Appl. Algebra 100 (1995), 173-216. 
[H4] Y.-Z. Huang, Vertex operator algebras and the Verlinde conjecture, Comm. Contemp. Math. 10 (2008), 103-154.

[H5] Y.-Z. Huang, Rigidity and modularity of vertex tensor categories, Comm. Contemp. Math. 10 (2008), 871-911.

[H6] Y.-Z. Huang, Cofiniteness conditions, projective covers and the logarithmic tensor product theory, J. Pure Appl. Alg. 213 (2009), 458-475.

[HL1] Y.-Z. Huang and J. Lepowsky, A theory of tensor product for module categories for a vertex operator algebra, I, Selecta Mathematica (New Series) 1 (1995), 699-756.

[HL2] Y.-Z. Huang and J. Lepowsky, A theory of tensor product for module categories for a vertex operator algebra, II, Selecta Mathematica (New Series) 1 (1995), 757-786.

[HL3] Y.-Z. Huang and J. Lepowsky, A theory of tensor product for module categories for a vertex operator algebra, III, J. Pure Appl. Algebra 100 (1995), 141-171.

[HLZ1] Y.-Z. Huang, J. Lepowsky and L. Zhang, Logarithmic tensor category theory for generalized modules for a conformal vertex algebra, I: Introduction and strongly graded algebras and their generalized modules, in: Conformal Field Theories and Tensor Categories, Proceedings of a Workshop Held at Beijing International Center for Mathematics Research, ed. C. Bai, J. Fuchs, Y.-Z. Huang, L. Kong, I. Runkel and C. Schweigert, Mathematical Lectures from Beijing University, Vol. 2, Springer, New York, 2014, 169-248.

[HLZ2] Y.-Z. Huang, J. Lepowsky and L. Zhang, Logarithmic tensor category theory, II: Logarithmic formal calculus and properties of logarithmic intertwining operators, arXiv:1012.4196.

[HLZ3] Y.-Z. Huang, J. Lepowsky and L. Zhang, Logarithmic tensor category theory, III: Intertwining maps and tensor product bifunctors, arXiv:1012.4197.

[HLZ4] Y.-Z. Huang, J. Lepowsky and L. Zhang, Logarithmic tensor category theory, IV: Constructions of tensor product bifunctors and the compatibility conditions, arXiv:1012.4198.

[HLZ5] Y.-Z. Huang, J. Lepowsky and L. Zhang, Logarithmic tensor category theory, V: Convergence condition for intertwining maps and the corresponding compatibility conditions, arXiv:1012.4199.

[HLZ6] Y.-Z. Huang, J. Lepowsky and L. Zhang, Logarithmic tensor category theory, VI: Expansion condition, associativity of logarithmic intertwining operators, and the associativity isomorphisms, arXiv:1012.4202. 
[HLZ7] Y.-Z. Huang, J. Lepowsky and L. Zhang, Logarithmic tensor category theory, VII: Convergence and extension properties and applications to expansion for intertwining maps, arXiv:1110.1929.

[HLZ8] Y.-Z. Huang, J. Lepowsky and L. Zhang, Logarithmic tensor category theory, VIII: Braided tensor category structure on categories of generalized modules for a conformal vertex algebra, arXiv:1110.1931.

[HY] Y.-Z. Huang and J. Yang, Logarithmic intertwining operators and associative algebras, J. Pure Appl. Alg. 216 (2011), 1467-1492.

[L] S. Lang, Elliptic functions, Graduate Texts in Mathematics, Vol. 112, SpringerVerlag, New York, 1987.

[LL] J. Lepowsky and H. Li, Introduction to Vertex Operator Algebras and Their Representations, Progress in Mathematics, VOl. 227, Birkhäuser, Boston, 2003.

[M1] A. Milas, Weak modules and logarithmic intertwining operators for vertex operator algebras, in Recent Developments in Infinite-Dimensional Lie Algebras and Conformal Field Theory, ed. S. Berman, P. Fendley, Y.-Z. Huang, K. Misra, and B. Parshall, Contemp. Math., Vol. 297, American Mathematical Society, Providence, RI, 2002, 201225.

[M2] A. Milas, Logaritmic intertwining operators and vertex operators, Comm. Math.Phys. 277 (2008), 497-529.

[Miy1] M. Miyamoto, Intertwining operators and modular invariance, preprint, arXiv:math.QA/0010180

[Miy2] M. Myiamoto, Modular invariance of vertex operator algebras satisfying $C_{2^{-}}$ cofiniteness, Duke Math. J. 122 (2004), 51-91.

[MS1] G. Moore and N. Seiberg, Classical and quantum conformal field theory, Comm. Math. Phys. 123 (1989), 177-254.

[MS2] G. Moore and N. Seiberg, Polynomial equations for rational conformal field theories, Phys. Lett. B212 (1988), 451-460.

[W] C. Weibel An Introduction to Homological Algebra, Cambridge Studies in Adv. Math., Vol 38, Cambridge University Press, Cambridge, 1994.

[Z] Y. Zhu, Modular invariance of characters of vertex operator algebras, J. Amer. Math. Soc. 9 (1996), 237-307.

Department of Mathematics, Rutgers University, 110 Frelinghuysen Rd., PiscatAWAY, NJ 08854-8019

E-mail address: francesco.fiordalisi@rutgers.edu 